\title{
The Global Structure of Simple Space-Times
}

\author{
Richard P. A. C. Newman \\ Department of Mathematics, Institute of Advanced Studies, Australian National University, GPO Box \\ 4, Canberra, A.C.T. 2601, Australia
}

\begin{abstract}
According to a standard definition of Penrose, a space-time admitting well-defined future and past null infinities $\mathscr{I}^{+}$and $\mathscr{I}^{-}$is asymptotically simple if it has no closed timelike curves, and all its endless null geodesics originate from $\mathscr{I}^{-}$and terminate at $\mathscr{I}^{+}$. The global structure of such space-times has previously been successfully investigated only in the presence of additional constraints. The present paper deals with the general case. It is shown that $\mathscr{I}^{+}$ is diffeomorphic to the complement of a point in some contractible open 3-manifold, the strongly causal region $\mathscr{I}_{0}^{+}$of $\mathscr{I}^{+}$is diffeomorphic to $\mathbb{S}^{2} \times \mathbb{R}$, and every compact connected spacelike 2 -surface in $\mathscr{I}^{+}$is contained in $\mathscr{I}_{0}^{+}$ and is a strong deformation retract of both $\mathscr{I}_{0}^{+}$and $\mathscr{I}^{+}$. Moreover the space-time must be globally hyperbolic with Cauchy surfaces which, subject to the truth of the Poincare conjecture, are diffeomorphic to $\mathbb{R}^{3}$.
\end{abstract}

\section{Introduction}

Consider a space-time which develops from initial data on an $\mathbb{R}^{3}$ Cauchy surface, and models an isolated, massive body. Suppose that the gravitational field strength is insufficient to cause collapse or to give rise to orbiting null geodesics akin to those at $r=3 m$ in Schwarzschild space-time. One may then reasonably assume that all endless null geodesics originate from a past null infinity $\mathscr{I}^{-}$and escape to a future null infinity $\mathscr{I}^{+}$. As the space-time evolves, $\mathscr{I}^{+}$is exposed to data on an increasingly large region of the Cauchy surface, and may be expected to respond by exhibiting increasingly complicated behaviour. What can be said about the general structure of $\mathscr{I}^{+}$, and about its global topology in particular?

In order to answer such questions, it is first necessary to specify more precisely the class of space-times to be considered. The only assumptions that will be necessary are that there are well-defined future and past null infinities $\mathscr{I}^{+}$and $\mathscr{I}^{-}$, that all endless null geodesics originate from $\mathscr{I}^{-}$and terminate at $\mathscr{I}^{+}$, and that there are no closed timelike curves. The existence of an $\mathbb{R}^{3}$ Cauchy surface can, subject to the truth of the Poincaré conjecture, be derived from these hypotheses. According to Penrose [1] one could, on physical grounds, assume that $\mathscr{I}^{+}$ 
always has the same topology as for Minkowski space, namely $\mathbb{S}^{2} \times \mathbb{R}$. He attempted to substantiate this view with a sketch of a proof that no other possibility could arise. But his argument is incorrect for reasons described later. Subsequently Geroch [2] gave a correct argument on the basis of a presupposition that $\mathscr{I}^{+}$ would be of the form $K \times \mathbb{R}$ for some compact 2-manifold $K$. A result of Hawking and Ellis [3] elaborated on Geroch's technique, presupposing instead that strong causality would hold at all points of $\mathscr{I}^{+}$and $\mathscr{I}^{-}$in the unphysical conformal completion of the space-time. It will be shown that a physical interpretation of strong causality at $\mathscr{I}^{-}$is that there is no null geodesic whose history can influence every event in the space-time. The corresponding interpretation of strong causality at $\mathscr{I}^{+}$is that there is no null geodesic whose history can be influenced by every such event. Unfortunately it is not clear that any reasonable constraints on the initial data would result in the conditions of either Geroch or Hawking and Ellis being fulfilled.

The present paper presupposes nothing about $\mathscr{I}^{+}$or $\mathscr{I}^{-}$, but seeks to determine what restrictions arise as consequences of the development of the initial data. Subject to the truth of the Poincaré conjecture it is concluded that the topology of $\mathscr{I}^{+}$may, in general, be described as the complement in $\mathbb{R}^{3}$ of the intersection of a sequence of cubes-with-handles, each of which is contained and deformable to a point in the interior of its predecessor. Apart from the trivial case realised by Minkowski space, for which $\mathscr{I}^{+}$and $\mathscr{I}^{-}$are both homeomorphic to the complement of a point in $\mathbb{R}^{3}$, such topologies are impossible to visualise. The associated space-time physics must be most intriguing.

Although the space-times considered here are accurately identified by Penrose's definition of asymptotic simplicity [1], in the case of a null conformal boundary, there is a need to introduce new terminology. This is primarily because Hawking and Ellis have given a definition of an asymptotically simple and empty space-time, now commonly accepted in the literature, which includes their condition of strong causality at $\mathscr{I}^{+}$and $\mathscr{I}^{-}$. But another reason is that the term "asymptotic simplicity" is inappropriate as a description of structure which involves global constraints. A space-time in which all endless null geodesics originate from a past null infinity $\mathscr{I}^{-}$and terminate at a future null infinity $\mathscr{I}^{+}$, and which contains no closed timelike curves, will henceforth be said to be simple. This objective of this paper is to identify the principal causal and topological properties of simple space-times.

\section{Notation and Terminology}

All manifolds are Hausdorff and paracompact. They are also $C^{\infty}$ unless otherwise stated. For a manifold-with-boundary $N$, the boundary and interior are denoted by $\partial N$ and $N:=N-\partial N$ respectively.

The image of a function $f: X \rightarrow Y$ is denoted by $|f|$, and its limit set by $L(f, Y)$. The positive and negative limit sets of a curve $\mu: \mathbb{R} \supset I \rightarrow Y$ are denoted by $L^{+}(\mu, Y)$ and $L^{-}(\mu, Y)$ respectively. Clearly $L(\mu, Y)=L^{+}(\mu, Y) \cup L^{-}(\mu, Y)$.

A space-time is a pair $(M, \mathbf{g})$, where $M$ is a connected 4-manifold and $\mathbf{g}$ is a smooth time-oriented Lorentzian metric on $M$. All causal curves in $(M, \mathbf{g})$ should be understood to be future-directed unless stated otherwise. A causal curve of the 
form $\mu: \mathbb{R} \supset[a, b] \rightarrow M$, whether future or past directed, is said to be a causal curve from $\mu(a) \in M$ to $\mu(b) \in M$. Also, if $\mu(a) \in \mathscr{A} \subset M$ and $\mu(b) \in \mathscr{B} \subset M$, then $\mu$ is said to be a causal curve from $\mathscr{A}$ to $\mathscr{B}$. Let $\mathscr{S}, \mathscr{T} \subset M$. Then $I^{+}(\mathscr{S}, \mathscr{T})$ (respectively $\left.J^{+}(\mathscr{S}, \mathscr{T})\right)$ denotes the set of all $p \in \mathscr{T}$ such that there is a timelike (causal) curve in $\mathscr{T}$ from $\mathscr{S}$ to $p$. Clearly, if $\mathscr{S} \cap \mathscr{T}$ is empty then so are $I^{+}(\mathscr{S}, \mathscr{T})$ and $J^{+}(\mathscr{S}, \mathscr{T})$. Sets $I^{-}(\mathscr{S}, \mathscr{T})$ and $J^{-}(\mathscr{S}, \mathscr{T})$ are defined analogously. A set $\mathscr{S} \subset M$ is said to be locally acausal if, for each $p \in \mathscr{S}$, there exists a neighbourhood $\mathscr{N}_{p}$ of $p$ in $M$ such that there is no non-degenerate causal curve from $\mathscr{S}$ to $\mathscr{S}$ in $\mathscr{N}_{p}$. For any set $\mathscr{T} \subset M$, the future boundary of $\mathscr{T}$ in $M$ is defined to be the set of all $q \in \overline{\mathscr{T}}$ admitting a neighbourhood $\mathscr{N}_{q}$ in $M$ such that $J^{+}\left(q, \mathcal{N}_{q}\right) \cap \overline{\mathscr{T}}=\{q\}$. The past boundary of $\mathscr{T}$ is defined analogously. Both the future and past boundaries of $\mathscr{T}$ are necessarily locally acausal.

A homeomorphism is denoted by $\approx$, and a diffeomorphism by $\approx_{\text {diff }}$. Neither should be assumed to respect additional structure. A group isomorphism is denoted by $\cong$, and a bundle equivalence by $\simeq$. Finally, coefficients for singular homology and cohomology modules are in the integers $\mathbb{Z}$, unless stated otherwise.

\section{Null Asymptotes}

A space-time may be equipped with future and past null infinities as follows.

Definition 3.1. A $C^{r}$ null asymptote of a space-time $(M, \mathbf{g})$ is a pair $(\tilde{M}, \tilde{\mathbf{g}})$, where $\tilde{M}$ is a $C^{\infty}$ 4-manifold-with-boundary extending $M$, and $\tilde{\mathbf{g}}$ is a $C^{r}$ Lorentzian metric on $\tilde{M}$, for some $r \geqq 0$, such that

(I) $\tilde{M}=M \cup \partial \tilde{M}$;

(II) $\tilde{\mathbf{g}} \mid \tilde{M}$ is conformal to $\mathbf{g}$;

(III) $\partial \tilde{M}$ is a null hypersurface of $(\tilde{M}, \tilde{\mathbf{g}})$;

(IV) each null geodesic of $(M, \mathbf{g})$ having an endpoint in $\tilde{M}$ at a point of $\partial \tilde{M}$ has infinite affine length with respect to $\mathbf{g}$.

Denote by $\mathscr{I}^{+}$(respectively $\mathscr{I}^{-}$) the set of all $p \in \partial \tilde{M}$ for which there exists a future- (respectively past-) directed causal curve $\mu:[0,1) \rightarrow M$ of $(M, \mathbf{g})$ having an endpoint at $p$ in $\tilde{M}$. Since $\partial \tilde{M}$ is $C^{\infty}$, for every $q \in \partial \tilde{M}$ there exists either a future- or past-directed causal curve $\tilde{v}:[0,1] \rightarrow \tilde{M}$ of $(\tilde{M}, \tilde{\mathbf{g}})$ such that $\tilde{v}([0,1)) \subset \tilde{M}-\partial \tilde{M}=M, \tilde{v}(1)=q$. Since condition (II) gives that the causal curves of $(M, \tilde{\mathbf{g}} \mid M)$ are precisely the causal curves of $(M, \mathbf{g})$ there follows $\partial \tilde{M}=\mathscr{I}^{+} \cup \mathscr{I}^{-}$. Condition (III) now implies that $\mathscr{I}^{+}$and $\mathscr{I}^{-}$are disjoint and relatively open in $\partial \tilde{M}$. Each of $\mathscr{I}^{+}=\partial \tilde{M}-\mathscr{I}^{-}$and $\mathscr{I}^{-}=\partial \tilde{M}-\mathscr{I}^{+}$is therefore a relatively open and closed submanifold of $\partial \tilde{M}$ and hence is a union of components of $\partial \tilde{M}$. Conditions (III) and (IV) justify their being termed the future and past null infinities of $(\tilde{M}, \tilde{\mathbf{g}})$ respectively. Through any point of $\mathscr{I}^{+}$(respectively $\left.\mathscr{I}^{-}\right)$there is a unique endless null curve of $(\tilde{M}, \tilde{\mathbf{g}})$ in $\mathscr{I}^{+}\left(\mathscr{I}^{-}\right)$called a generator of $\mathscr{I}^{+}\left(\mathscr{I}^{-}\right)$. By (I) and (II), if $\tilde{\mathbf{g}}$ is $C^{r}$ for $r \geqq 1$, then a curve $\tilde{\sigma}: \mathbb{R} \supset I \rightarrow \tilde{M}$ is a null geodesic of $(\tilde{M}, \tilde{\mathbf{g}})$ iff $\tilde{\sigma} \mid \stackrel{\circ}{I}$ is a null geodesic of $(M, \mathbf{g})$ or $\tilde{\sigma}$ is a generating segment of $\mathscr{I}^{+}$or $\mathscr{I}^{-}$. In the case $r=0$, the null geodesics of $(\tilde{M}, \tilde{\mathbf{g}})$ may be sensibly defined by the requirement that the same is true. 
The presence of a boundary to $\tilde{M}$ complicates the causal structure of $(\tilde{M}, \tilde{\mathbf{g}})$. Nonetheless there are two basic results for space-times-without-boundary which carry over directly:

(1) for any set $\mathscr{S} \subset \tilde{M}$ the set $I^{+}(\mathscr{S}, \tilde{M})$ is open in $\tilde{M}$;

(2) if there is a causal curve from $x \in \tilde{M}$ to $y \in \tilde{M}$ which is not a null geodesic, then there is a timelike curve from $x$ to $y$.

The proofs are adaptations of the corresponding proofs for space-times without boundary. Note that neither (1) nor (2) is true for general space-times-withboundary.

Lemma 3.2. $\mathscr{I}^{ \pm}=\partial \tilde{M} \cap I^{ \pm}(\tilde{M}, \tilde{M})=\partial \tilde{M}-I^{\mp}(\tilde{M}, \tilde{M})$.

Proof. Immediately by (2) and since $\mathscr{I}^{+}$and $\mathscr{I}^{-}$are null.

For the space-time $(M, \mathbf{g})$ and any set $\mathscr{S} \subset M$ one has $\bar{I}^{+}(\mathscr{S}, M)=\bar{J}^{+}(\mathscr{S}, M)$ and $\dot{I}^{+}(\mathscr{S}, M)=\dot{J}^{+}(\mathscr{S}, M)$. For the null asymptote $(\tilde{M}, \tilde{\mathbf{g}})$ the corresponding results are less straightforward.

Lemma 3.3. If $\mathscr{S} \subset \tilde{M}-\mathscr{I}^{+}$, then

(I) $\bar{I}^{+}(\mathscr{S}, \tilde{M})=\bar{J}^{+}(\mathscr{S}, \tilde{M})$;

(II) $\dot{I}^{+}(\mathscr{S}, \tilde{M})=\dot{J}^{+}(\mathscr{S}, \tilde{M}) \cup J^{+}\left(\mathscr{S}, \mathscr{I}^{-}\right)$.

Proof.

(I) The inclusion $I^{+}(\mathscr{S}, \tilde{M}) \subset J^{+}(\mathscr{S}, \tilde{M})$ implies $\bar{I}^{+}(\mathscr{S}, \tilde{M}) \subset \bar{J}^{+}(\mathscr{S}, \tilde{M})$. For the converse, let $p \in \bar{J}^{+}(\mathscr{S}, \tilde{M})$ and let $\mathscr{N}_{p}$ be an open neighbourhood of $p$ in $\tilde{M}$. Since $\mathscr{N}_{p}$ intersects $J^{+}(\mathscr{S}, \tilde{M})$ there exists a causal curve $\mu$ from some $x \in \mathscr{S}$ to some $y \in \mathscr{N}_{p}$. If $y \notin \mathscr{I}^{+}$there exists $z \in I^{+}\left(y, \mathscr{N}_{p}\right) \subset I^{+}(\mathscr{S}, \tilde{M}) \cap \mathscr{N}_{p}$. If $y \in \mathscr{I}^{+}$there exists a non-degenerate generating segment $v$ of $\mathscr{I}^{+}$in $\mathscr{N}_{p}$ from $y$ to some $z \in \mathscr{I}^{+} \cap \mathscr{N}_{p}$. Since the concatenation of $\mu$ and $v$ is a causal curve from $x \in \mathscr{S} \subset \tilde{M}-\mathscr{I}^{+}$through $y \in \mathscr{I}^{+}$to $z \in \mathscr{I}^{+}$, and cannot therefore be a null geodesic, one again has $z \in I^{+}(\mathscr{S}, \tilde{M}) \cap \mathscr{N}_{p}$. There follows $p \in \bar{I}^{+}(\mathscr{S}, \tilde{M})$.

(II) Let $p \in \dot{J}^{+}(\mathscr{S}, \tilde{M})$. Then $p \in \bar{J}^{+}(\mathscr{S}, \tilde{M})=\bar{I}^{+}(\mathscr{S}, \tilde{M})$ and every neighbourhood of $p$ intersects $I^{+}(\mathscr{S}, \tilde{M})$. Since $I^{+}(\mathscr{S}, \tilde{M})$ is open one cannot have $p \in I^{+}(\mathscr{S}, \tilde{M})$ otherwise there would exist a neighbourhood of $p$ contained in $I^{+}(\mathscr{S}, \tilde{M}) \subset$ $J^{+}(\mathscr{S}, \tilde{M})$ and $p$ would be an interior point of $J^{+}(\mathscr{S}, \tilde{M})$. Hence $p \in \tilde{M}-I^{+}(\mathscr{S}, \tilde{M})$ and consequently $\dot{J}^{+}(\mathscr{S}, \tilde{M}) \subset \dot{I}^{+}(\mathscr{S}, \tilde{M})$.

Let $p \in J^{+}\left(\mathscr{S}, \mathscr{I}^{-}\right)$. Then for every open neighbourhood $\mathscr{N}_{p}$ of $p$ there exists $p^{\prime} \in I^{+}\left(p, \mathscr{N}_{p}\right) \subset I^{+}(\mathscr{S}, \tilde{M}) \cap \mathscr{N}_{p}$. Since $I^{+}(\mathscr{S}, \tilde{M})$ does not intersect $\mathscr{I}^{-}$one has $p \in \tilde{M}-I^{+}(\mathscr{S}, \tilde{M})$ and there follows $p \in \dot{I}^{+}(\mathscr{S}, \tilde{M})$. Thus $J^{+}\left(\mathscr{S}, \mathscr{I}^{-}\right) \subset \dot{I}^{+}(\mathscr{S}, \tilde{M})$.

Let $q \in \dot{I}^{+}(\mathscr{S}, \tilde{M})-\mathscr{I}^{-}$and let $\mathscr{N}_{q}$ be an open neighbourhood of $q$ in $\tilde{M}_{\tilde{M}}$. Then $\mathscr{N}_{q}$ intersects $I^{+}(\mathscr{S}, \tilde{M})$ and therefore intersects $J^{+}(\mathscr{S}, \tilde{M})$. If $\mathscr{N}_{q} \subset J^{+}(\mathscr{S}, \tilde{M})$ then for any $q^{-} \in I^{-}\left(q, \mathscr{N}_{q}\right)$ one would have $q \in I^{+}\left(q^{-}, \mathscr{N}_{q}\right) \subset I^{+}(\mathscr{\mathscr { S }}, \tilde{M})$. Since this is incompatible with $q \in \dot{I}^{+}(\mathscr{S}, \tilde{M}), \mathscr{N}_{q}$ must intersect $\tilde{M}-J^{+}(\mathscr{S}, \tilde{M})$. There follows $q \in \dot{J}^{+}(\mathscr{S}, \tilde{M})$ and hence $\dot{I}^{+}(\mathscr{S}, \tilde{M})-\mathscr{I}^{-} \subset J^{+}(\mathscr{S}, \tilde{M})$.

If $r \in\left(\dot{I}^{+}(\mathscr{S}, \tilde{M}) \cap \mathscr{I}^{-}\right)-J^{+}(\mathscr{S}, \tilde{M})$, then every neighbourhood of $r$ intersects $I^{+}(\mathscr{S}, \tilde{M})$ and therefore intersects $J^{+}(\mathscr{S}, \tilde{M})$. Since $J^{+}(\mathscr{S}, \tilde{M})$ does not contain $r$ one therefore has $r \in \dot{J}^{+}(\mathscr{S}, \tilde{M})$. Hence $\left(\dot{I}^{-}(\mathscr{S}, \tilde{M}) \cap \mathscr{I}^{-}\right)-J^{+}(\mathscr{S}, \tilde{M}) \subset J^{+}(\mathscr{S}, \tilde{M})$ 
and consequently $\dot{I}^{+}(\mathscr{S}, \tilde{M}) \cap \mathscr{I}^{-} \subset \dot{J}^{+}(\mathscr{S}, \tilde{M}) \cup\left(J^{+}(\mathscr{S}, \tilde{M}) \cap \mathscr{I}^{-}\right)=\dot{J}^{+}(\mathscr{S}, \tilde{M}) \cup$ $J^{+}\left(\mathscr{S}, \mathscr{I}^{-}\right)$.

One now has $\dot{I}^{+}(\mathscr{S}, \tilde{M})=\dot{J}^{+}(\mathscr{S}, \tilde{M}) \cup J^{+}\left(\mathscr{S}, \mathscr{I}^{-}\right)$as required.

Corollary. If $\mathscr{S} \subset M$ then $\bar{I}^{+}(\mathscr{S}, \tilde{M})=\bar{J}^{+}(\mathscr{S}, \tilde{M})$ and $\dot{I}^{+}(\mathscr{S}, \tilde{M})=\dot{J}^{+}(\mathscr{S}, \tilde{M})$.

In order to see the necessity for the restriction $\mathscr{S} \subset \tilde{M}-\mathscr{I}^{+}$in Lemma 3.3, observe that for $\mathscr{S}=\{p\}, p \in \mathscr{I}^{+}$, the set $I^{+}(\mathscr{S}, \tilde{M})$ is empty, whilst both $J^{+}(\mathscr{S}, \tilde{M})$ and $J(\mathscr{S}, \tilde{M})$ are non-empty. In this case therefore, neither (I) nor (II) hold.

A future set in $(M, \mathbf{g})$ is conventionally defined as a set $\mathscr{F} \subset M$ such that $I^{+}(\mathscr{F}, M) \subset \mathscr{F}$. For the purposes of this paper, the slightly more restrictive condition $J^{+}(\mathscr{F}, M) \subset \mathscr{F}$ is imposed. A future set in $(\tilde{M}, \tilde{\mathbf{g}})$ is defined analogously. If $\mathscr{F}$ is a future set of $(M, \mathbf{g})$ then so is $\overline{\mathscr{F}}$, with a similar result holding for $(\tilde{M}, \tilde{\mathbf{g}})$. For any future set $\mathscr{F}$ of $(M, \mathbf{g})$ one has that $\dot{\mathscr{F}}$ is a closed achronal embedded topological 3-submanifold of $M$. The following is the corresponding result for $(\tilde{M}, \tilde{\mathbf{g}})$.

Lemma 3.4. Let $\mathscr{F}$ be a future set of $(\tilde{M}, \tilde{\mathbf{g}})$ such that $\dot{\mathscr{F}} \cap \mathscr{I}^{+}$and $\dot{\mathscr{F}} \cap \mathscr{I}^{-}$are acausal. Then $\dot{\mathscr{F}}, \overline{\mathscr{F}} \cap \mathscr{I}^{+}$and $\overline{\mathscr{F}} \cap \mathscr{I}^{-}$are closed achronal embedded topological 3-submanifolds-with-boundary of $\tilde{M}$ such that $\partial \dot{\mathscr{F}}=\partial\left(\overline{\mathscr{F}} \cap \mathscr{I}^{+}\right) \cup \partial\left(\overline{\mathscr{F}} \cap \mathscr{I}^{-}\right)=\dot{\mathscr{F}} \cap \partial \tilde{M}$.

Proof. Since $\tilde{M}$ is Hausdorff and paracompact, the sets $\dot{\mathscr{F}}, \overline{\mathscr{F}} \cap \mathscr{I}^{+}$and $\overline{\mathscr{F}} \cap \mathscr{I}^{-}$ are Hausdorff and paracompact in their relative topologies. Clearly $\dot{\mathscr{F}}, \overline{\mathscr{F}} \cap \mathscr{I}^{+}$ and $\overline{\mathscr{F}} \cap \mathscr{I}^{-}$are closed and achronal in $(\tilde{M}, \tilde{\mathbf{g}})$.

Let $q \in \dot{\mathscr{F}}-\partial \tilde{M}$, let $\mathscr{N}$ be a globally hyperbolic open neighbourhood of $q$ in $M$, and let $\mathscr{H}$ be a Cauchy surface for $\mathscr{N}$ such that $q \in \mathscr{H}$. Let $\mathbf{X}$ be a timelike vector field on $\mathscr{N}$. The integral curves of $\mathbf{X}$ define a continuous open mapping $\phi: \mathscr{N} \rightarrow \mathscr{H}$. The restriction $\psi:=\phi \mid \dot{\mathscr{F}} \cap \mathscr{N}$ is a continuous injection. Let $\mathscr{U}^{\prime} \subset \mathscr{N}$ be open in $M$ and have non-empty intersection with $\dot{\mathscr{F}}$. Let $q^{\prime} \in \psi\left(\dot{\mathscr{F}} \cap \mathscr{U}^{\prime}\right) \subset \mathscr{H}$. Let $\mathscr{V}^{\prime} \subset \mathscr{U}^{\prime}$ be an open neighbourhood of $\psi^{-1}\left(q^{\prime}\right) \in \dot{\mathscr{F}} \cap \mathscr{U}^{\prime}$ in $M$ such that the only integral curves of $\mathbf{X} \mid \mathscr{N}$ from $\mathscr{V}^{\prime}$ to $\mathscr{V}^{\prime}$ are those in $\mathscr{V}^{\prime}$. There exists an open neighbourhood $\mathscr{W}^{\prime} \subset \mathscr{V}^{\prime}$ of $\psi^{-1}\left(q^{\prime}\right)$ in $M$ such that every maximal integral curve of $\mathbf{X} \mid \mathscr{N}$ which cuts $\mathscr{W}^{\prime}$ also cuts $I^{+}\left(\psi^{-1}\left(q^{\prime}\right), \mathscr{V}^{\prime}\right) \subset \mathscr{F} \cap \mathscr{V}^{\prime}$ and $I^{-}\left(\psi^{-1}\left(q^{\prime}\right), \mathscr{V}^{\prime}\right) \subset \mathscr{V}^{\prime}-\mathscr{F}$. Every such integral curve therefore cuts $\dot{\mathscr{F}} \cap \mathscr{V}^{\prime} \subset \dot{\mathscr{F}} \cap \mathscr{U}^{\prime}$ and it follows that $\psi\left(\dot{\mathscr{F}} \cap \mathscr{U}^{\prime}\right)$ contains the relative open neighbourhood $\phi\left(\mathscr{W}^{\prime}\right)$ of $q^{\prime}$ in $\mathscr{H}$. Thus $\psi\left(\dot{\mathscr{F}} \cap \mathscr{U}^{\prime}\right)$ is a relative open neighbourhood in $\mathscr{H}$ of each of its points and so is relatively open in $\mathscr{H}$. One now has that $\psi: \dot{\mathscr{F}} \cap \mathscr{N} \rightarrow \mathscr{H}$ is an open continuous injection, and therefore a homeomorphism, onto $\psi(\dot{\mathscr{F}} \cap \mathscr{N})$ which must be a relative open neighbourhood of $q$ in $\mathscr{H}$. For any relative open neighbourhood $\mathscr{O} \approx \mathbb{R}^{3}$ of $q$ in $\psi(\dot{\mathscr{F}} \cap \mathscr{N})$ the set $\psi^{-1}(\mathcal{O}) \approx \mathbb{R}^{3}$ is a relative open neighbourhood of $q$ in $\mathscr{\mathscr { F }} \cap \mathcal{N}$, and therefore in $\dot{\mathscr{F}}-\partial \tilde{M}$. Thus $\dot{\mathscr{F}}-\partial \tilde{M}$ is a topological 3-manifold. Moreover, since $\partial \tilde{M}$ is closed in $\tilde{M}$, the set $\dot{\mathscr{F}} \cap \partial \tilde{M}$ is relatively closed in $\dot{\mathscr{F}}$, and so the pair $(\dot{\mathscr{F}}, \dot{\mathscr{F}} \cap \partial \tilde{M})$ is a relative topological 3-manifold (Spanier [4, p. 297]).

Let $\mathbf{Y}$ be a nowhere-zero vector field on $\mathscr{I}^{+}$such that each integral curve of $\mathbf{Y}$ is a future-directed generating segment of $\mathscr{I}^{+}$. Let $q \in \dot{\mathscr{F}} \cap \mathscr{I}^{+}$and let $\mathscr{N}$ be a relative open neighbourhood of $q$ in $\mathscr{I}^{+}$admitting an embedded 2-submanifold $\mathscr{H} \ni q$ such that each maximal integral curve of $\mathbf{Y} \mid \mathcal{N}$ cuts $\mathscr{H}$ at a single point. These curves define a continuous open mapping $\phi: \mathscr{N} \rightarrow \mathscr{H}$. The restriction $\psi:=\phi \mid \dot{\mathscr{F}} \cap \mathcal{N}$ is a continuous injection. Let $\mathscr{U}^{\prime} \subset \mathscr{N}$ be relatively open in $\mathscr{I}^{+}$and 
have non-empty intersection with $\dot{\mathscr{F}}$. Let $q^{\prime} \in \psi\left(\dot{\mathscr{F}} \cap \mathscr{U}^{\prime}\right) \subset \mathscr{H}$. Let $\mathscr{V}^{\prime} \subset \mathscr{U}^{\prime}$ be a relative open neighbourhood of $\psi^{-1}\left(q^{\prime}\right) \in \dot{\mathscr{F}} \cap \mathscr{N}$ in $\mathscr{I}^{+}$such that the only integral curves of $\mathbf{Y} \mid \mathscr{N}$ from $\mathscr{V}^{\prime}$ to $\mathscr{V}^{\prime}$ are those in $\mathscr{V}^{\prime}$. For any integral curve $\mu:[-\delta, \delta] \rightarrow \mathscr{V}^{\prime}$ of $\mathbf{Y}$ such that $\mu(0)=\psi^{-1}\left(\mathbf{q}^{\prime}\right)$, the hypothesis that $\dot{\mathscr{F}} \cap \mathscr{I}^{+}$and $\dot{\mathscr{F}} \cap \mathscr{I}^{-}$are acausal implies $\mu(u) \in \operatorname{int}(\mathscr{F})$ for all $u \in(0, \delta]$ and $\mu(u) \in M-\overline{\mathscr{F}}$ for all $u \in[-\delta, 0)$. It follows that there exists a relative open neighbourhood $\mathscr{W}^{\prime} \subset \mathscr{V}^{\prime}$ of $\psi^{-1}\left(q^{\prime}\right)$ in $\mathscr{I}^{+}$such that every maximal integral curve of $\mathbf{Y} \mid \mathscr{N}$ which cuts $\mathscr{W}^{\prime}$ also cuts both int $(\mathscr{F}) \cap \mathscr{V}^{\prime}$ and $\mathscr{V}^{\prime}-\overline{\mathscr{F}}$. Every such curve therefore cuts $\dot{\mathscr{F}} \cap \mathscr{V}^{\prime} \subset \dot{\mathscr{F}} \cap \mathscr{U}^{\prime}$ and it follows that $\psi\left(\dot{\mathscr{F}} \cap \mathscr{U}^{\prime}\right)$ contains the relative open neighbourhood $\phi\left(\mathscr{W}^{\prime}\right)$ of $q^{\prime}$ in $\mathscr{H}$. Thus $\psi\left(\dot{\mathscr{F}} \cap \mathscr{U}^{\prime}\right)$ is a relative open neighbourhood of each of its points and so is relatively open in $\mathscr{H}$. Hence $\psi: \dot{\mathscr{F}} \cap \mathscr{N} \rightarrow \mathscr{H}$ is an open continuous injection, and therefore a homeomorphism, onto $\psi(\dot{\mathscr{F}} \cap \mathscr{N})$ which must be a relative open neighbourhood of $q$ in $\mathscr{H}$. Since $\mathscr{H}$ is a topological 2-manifold it follows that there exists a relative open neighbourhood of $q$ in $\dot{\mathscr{F}} \cap \mathscr{N} \subset \dot{\mathscr{F}} \cap \mathscr{I}^{+}$homeomorphic to $\mathbb{R}^{2}$. Thus $\dot{\mathscr{F}} \cap \mathscr{I}^{+}$ is a topological 2-submanifold of $\mathscr{I}^{+}$. Similarly, since $\mathscr{P}:=\tilde{M}-\mathscr{F}$ is a past set of $(\tilde{M}, \tilde{\mathbf{g}})$, the acausal set $\dot{\mathscr{F}} \cap \mathscr{I}^{-}=\dot{\mathscr{P}} \cap \mathscr{I}^{-}$is a topological 2-submanifold of $\mathscr{I}^{-}$.

Let $r \in \dot{\mathscr{F}} \cap \mathscr{I}^{+}$and let $\mathscr{R} \approx \mathbb{R}^{2}$ be a relative open neighbourhood of $r$ in $\dot{\mathscr{F}} \cap \mathscr{I}^{+}$, with compact closure in $\mathscr{I}^{+}$. There exists a topological embedding $\Phi: \mathscr{R} \times(-\varepsilon, \varepsilon) \rightarrow \mathscr{I}^{+}$, for some $\varepsilon>0$, such that $\Phi(s, 0)=s$ for all $s \in \mathscr{R}$, with each $\mu_{s}:=\Phi(s, \cdot):(-\varepsilon, \varepsilon) \rightarrow \mathscr{I}^{+}$an integral curve of $\mathbf{Y}$. Invariance of domain gives that $\mathscr{N}:=\Phi(\mathscr{R} \times(-\varepsilon, \varepsilon))$ is relatively open in $\mathscr{I}^{+}$, so $\mathscr{N} \cap \overline{\mathscr{F}}$ is relatively open in $\overline{\mathscr{F}} \cap \mathscr{I}^{+}$. For each $s \in \mathscr{R}$ one has $\mu_{s}(u) \in \operatorname{int}(\mathscr{F})$ for all $u \in(0, \varepsilon), \mu_{s}(u) \in \mathscr{I}^{+}-\overline{\mathscr{F}}$ for all $u \in(-\varepsilon, 0)$, and hence $\mathscr{N} \cap \overline{\mathscr{F}}=\Phi(\mathscr{R} \times[0, \varepsilon))$. There follows $\mathscr{N} \cap \overline{\mathscr{F}} \approx$ $\mathscr{R} \times[0, \varepsilon) \approx \frac{1}{2} \mathbb{R}^{3}$. This shows that $\overline{\mathscr{F}} \cap \mathscr{I}^{+}$is a topological 3-submanifold-withboundary of $\mathscr{I}^{+}$such that $\partial\left(\overline{\mathscr{F}} \cap \mathscr{I}^{+}\right)=\dot{\mathscr{F}} \cap \mathscr{I}^{+}$. Similarly $\overline{\mathscr{F}} \cap \mathscr{I}^{-}$is a topological 3-submanifold-with-boundary of $\mathscr{I}^{-}$such that $\partial\left(\overline{\mathscr{F}} \cap \mathscr{I}^{-}\right)=\dot{\mathscr{F}} \cap \mathscr{I}^{-}$.

Let $\widetilde{\mathbf{Z}}$ be a timelike vector field on $\tilde{M}$. Let $q \in \dot{\mathscr{F}} \cap \mathscr{I}^{+}$and let $\mathscr{N} \subset \tilde{M}-\mathscr{I}^{-}$be an open neighbourhood of $q$ in $\tilde{M}$ such that each point of $\mathscr{N}$ may be connected to a point of $\mathscr{N} \cap \mathscr{I}^{+}$by an integral curve of $\tilde{\mathbf{Z}} \mid \mathscr{N}$. The integral curves of $\tilde{\mathbf{Z}} \mid \mathscr{N}$ define a continuous open mapping $\phi: \mathscr{N} \rightarrow \mathscr{N} \cap \mathscr{I}^{-}$. Since $\overline{\mathscr{F}}$ is a future set of $(\tilde{M}, \tilde{\mathbf{g}})$ one has $\phi(\dot{\mathscr{F}} \cap \mathscr{N}) \subset \dot{\mathscr{F}}$. The restriction $\psi:=\phi \mid \dot{\mathscr{F}} \cap \mathscr{N}$ is therefore a continuous injection into $\overline{\mathscr{F}} \cap \mathscr{I}^{+}$. Let $\mathscr{U}^{\prime} \subset \mathscr{N}$ be open in $\tilde{M}$ and have non-empty intersection with $\dot{\mathscr{F}}$. Let $q^{\prime} \in \psi\left(\dot{\mathscr{F}} \cap \mathcal{N}^{\prime}\right) \subset \dot{\mathscr{F}} \cap \mathscr{I}^{+}$. If $\psi^{-1}\left(q^{\prime}\right) \in M$ then $q^{\prime} \in \operatorname{int}(\mathscr{F})$ and arguments analogous to those employed previously give that $\psi^{-1}\left(q^{\prime}\right)$ admits an open neighbourhood $\mathscr{W}^{\prime} \subset \mathscr{U}^{\prime}$ such that $\phi\left(\mathscr{W}^{\prime}\right) \subset \psi\left(\dot{\mathscr{F}} \cap \mathscr{U}^{\prime}\right) \cap \operatorname{int}(\mathscr{F})$. Then $\psi\left(\dot{\mathscr{F}} \cap \mathscr{U}^{\prime}\right)$ contains the relative open neighbourhood $\phi\left(\mathscr{W}^{\prime}\right) \cap \overline{\mathscr{F}}=\phi\left(\mathscr{W}^{\prime}\right)$ of $q^{\prime}$ in $\overline{\mathscr{F}} \cap \mathscr{I}^{+}$. Now suppose $\psi^{-1}\left(q^{\prime}\right)=q^{\prime} \in \mathscr{I}^{+}$. In this case let $\mathscr{W}^{\prime} \subset \mathscr{U}^{\prime}$ be an open neighbourhood of $\psi^{-1}\left(q^{\prime}\right)$ in $\tilde{M}$ such that every maximal integral curve of $\tilde{\mathbf{Z}} \mid \mathscr{N}$ which cuts $\mathscr{W}^{\prime}$ also cuts $I^{-}\left(q^{\prime}, \mathscr{N}\right)$ and has a future endpoint in $\mathscr{I}^{+} \cap \mathscr{W}^{\prime}$. Let $y \in \phi\left(\mathscr{W}^{\prime}\right)$. If $y \notin \psi\left(\dot{\mathscr{F}} \cap \mathscr{W}^{\prime}\right)$ then the maximal integral curve $\mu_{y}$ of $\widetilde{\mathbf{Z}} \mid \mathscr{W}^{\prime}$ to $y$ does not cut $\dot{\mathscr{F}}$. Since $\mu_{y}$ cuts $I^{-}\left(q^{\prime}, \mathscr{N}\right) \subset \tilde{M}-\overline{\mathscr{F}}$ one has $y \notin \overline{\mathscr{F}}$ and thus $\psi\left(\dot{\mathscr{F}} \cap \mathscr{W}^{\prime}\right) \supset$ $\phi\left(\mathscr{W}^{\prime}\right) \cap \overline{\mathscr{F}}$. Hence in this case $\psi\left(\dot{\mathscr{F}} \cap \mathscr{U}^{\prime}\right) \supset \psi\left(\dot{\mathscr{F}} \cap \mathscr{W}^{\prime}\right)$ contains the relative open neighbourhood $\phi\left(\mathscr{W}^{\prime}\right) \cap \overline{\mathscr{F}}$ of $q^{\prime}$ in $\overline{\mathscr{F}} \cap \mathscr{I}^{+}$. In general therefore, $\psi\left(\dot{\mathscr{F}} \cap \mathscr{U}^{\prime}\right)$ is a relative neighbourhood in $\overline{\mathscr{F}} \cap \mathscr{I}^{+}$of each of its points and is therefore relatively open in $\overline{\mathscr{F}} \cap \mathscr{I}^{+}$. Hence $\psi: \dot{\mathscr{F}} \cap \mathscr{N} \rightarrow \overline{\mathscr{F}} \cap \mathscr{I}^{+}$is an open continuous injection, and therefore a homeomorphism, onto $\psi(\dot{\mathscr{F}} \cap \mathscr{N})$ which must be relatively open in 
$\overline{\mathscr{F}} \cap \mathscr{I}^{+}$. Since $\overline{\mathscr{F}} \cap \mathscr{I}^{+}$is a topological 3-manifold-with-boundary, the point $q=\psi(q) \in \dot{\mathscr{F}} \cap \mathscr{I}^{+}=\partial\left(\overline{\mathscr{F}} \cap \mathscr{I}^{+}\right)$admits a relative open neighbourhood $\mathcal{O} \approx \frac{1}{2} \mathbb{R}^{3}$ in $\overline{\mathscr{F}} \cap \mathscr{I}^{+}$contained in $\mathscr{N} \cap \mathscr{I}^{+}$. The set $\psi^{-1}(\mathcal{O}) \approx \frac{1}{2} \mathbb{R}^{3}$ is a relative open neighbourhood of $q \in \dot{\mathscr{F}} \cap \mathscr{I}^{+}$in $\dot{\mathscr{F}} \cap \mathscr{N}$, and therefore in $\dot{\mathscr{F}}$. Similarly each $q \in \dot{\mathscr{F}} \cap \mathscr{I}^{-}$has a relative open neighbourhood in $\dot{\mathscr{F}}$ homeomorphic to $\frac{1}{2} \mathbb{R}^{3}$. It follows that $\dot{\mathscr{F}}$ is a 3 -submanifold-with-boundary of $\tilde{M}$ such that $\partial \dot{\mathscr{F}}=$ $\left(\dot{\mathscr{F}} \cap \mathscr{I}^{+}\right) \cup\left(\dot{\mathscr{F}} \cap \mathscr{I}^{-}\right)=\dot{\mathscr{F}} \cap \partial \tilde{M}$.

In order to see the necessity of the hypothesis that $\dot{\mathscr{F}} \cap \mathscr{I}^{+}$and $\dot{\mathscr{F}} \cap \mathscr{I}^{-}$are acausal in this result, observe that for any $p \in \mathscr{I}^{+}$, the future set $\mathscr{F}=J^{+}(p, \tilde{M})$ of $(\tilde{M}, \tilde{\mathbf{g}})$ is such that $\dot{\mathscr{F}}=\mathscr{F} \subset \mathscr{I}^{+}$is a non-acausal immersed 1-submanifold-withboundary of $\tilde{M}$ and need not be closed.

According to Penrose [5], strong causality is said to hold at a point $p \in M$ iff every neighbourhood $\mathcal{N}$ of $p$ in $M$ contains a neighbourhood $\mathscr{N}^{\prime}$ of $p$ in $M$ such that the only causal curves of $(M, \mathbf{g})$ from $\mathscr{N}^{\prime}$ to $\mathscr{N}^{\prime}$ are those in $\mathscr{N}^{\prime}$. For present purposes however, strong causality is defined as follows.

Definition 3.5. Strong causality holds at a point $p$ if every neighbourhood $\mathscr{N}$ of $p$ contains a neighbourhood $\mathscr{N}^{\prime}$ of $p$ such that the only causal curves from $\mathscr{N}^{\prime}$ to $\mathscr{N}^{\prime}$ are those in $\mathscr{N}$.

Clearly strong causality at $p$ in the sense of Penrose implies strong causality in the sense of Definition 3.5. Conversely, in the space-time $(M, \mathbf{g})$, the existence of convex normal neighbourhoods may be invoked to show that strong causality at $p$ in the sense of Definition 3.5 implies strong causality in the sense of Penrose. The argument may be adapted to apply to $(\tilde{M}, \tilde{\mathbf{g}})$. Definition 3.5 has the advantage that it does not depend upon the existence of convex normal neighbourhoods, or their analogues for null asymptotes, to ensure that strong causality relates purely to global structure. It is consequently easier to work with.

In the space-time $(M, \mathbf{g})$, if strong causality holds at every point of a compact set $\mathscr{K} \subset M$, then $M \not I^{-}(\mathscr{K}, M)$. For the null assumptote $(\tilde{M}, \tilde{\mathbf{g}})$ the corresponding result is as follows.

Lemma 3.6. Let $\mathscr{K} \subset \tilde{M}$ be compact. If strong causality holds at every point of $\mathscr{K}$ in $(\tilde{M}, \tilde{\mathbf{g}})$ then $M \not I^{-}(\mathscr{K}, M)$.

Proof. Suppose $M \subset I^{-}(\mathscr{K}, \tilde{M})$ and also that strong causality holds at every point of $\mathscr{K}$ in $\tilde{M}$. Since the strongly causal region of $\tilde{M}$ is open, there exists a compact neighbourhood $\mathscr{N}$ of $\mathscr{K}$ in $\tilde{M}$ such that strong causality holds at every point of $\mathscr{N}$. Let $\mathscr{N}_{i} \subset \mathscr{N}$ be a decreasing sequence of open neighbourhoods of $\mathscr{K}$ in $\tilde{M}$ such that $\bigcap_{i} \mathscr{N}_{i}=\mathscr{K}$. One has $M \subset I^{-}\left(\mathscr{N}_{i}, \tilde{M}\right)$ for each $i$. Choose $q_{1} \in \mathscr{N}_{1} \cap M$.

Since $\mathscr{N}$ cannot totally future imprison any future endless causal curve of $(\tilde{M}, \tilde{\mathbf{g}})$, there exists a causal curve of $(\tilde{M}, \tilde{\mathbf{g}})$ from $q_{1} \in M$ to the open set $\tilde{M}-\mathscr{N}$. It follows that there exists a timelike curve from $q_{1}$ to $\tilde{M}-\mathcal{N}$, and hence a timelike curve $\lambda_{1}^{-}$ from $q_{1}$ to some $r_{1} \in M-\mathscr{N}$. The inclusion $M \subset I^{-}\left(\mathscr{N}_{2}, \tilde{M}\right)$ now gives that there exists a timelike future extension $\lambda_{1}^{+}$of $\lambda_{1}^{-}$from $q_{1}$ through $r_{1} \in M-\mathscr{N}$ to some $q_{2}^{+} \in \mathscr{N}_{2}$. Since $\mathscr{N}_{2}$ is open, $\lambda_{1}^{+}$admits a segment $\lambda_{1}$ from $q_{1}$ through $r_{1}$ to some $q_{2} \in \mathscr{N}_{2} \cap M$. Continue inductively to find, for each $i$, a point $q_{i} \in \mathscr{N}_{i} \cap M \subset \mathscr{N}$ 
and a timelike curve $\lambda_{i}$ from $q_{i}$ to $q_{i+1}$ which cuts $\tilde{M}-\mathscr{N}$. Since $\mathscr{N}$ is compact, the $q_{i}$ admit a cluster point $q \in \mathscr{N}$. In fact the condition $\bigcap_{i} \mathscr{N}_{i}=\mathscr{K}$ ensures $q \in \mathscr{K}$. The concatenation of the $\lambda_{i}$ is a future endless timelike curve $\lambda$ from $q_{1}$ such that $q \in L^{+}(\lambda, \tilde{M}) \cap \mathscr{K}$. It follows that strong causality is violated at $q \in \mathscr{K}$ and one has a contradiction.

\section{Simple Space-Times and Their Causal Structure}

The class of space-times of central interest in this paper are identified by the following.

Definition 4.1. Let $(M, \mathbf{g})$ be a space-time satisfying the chronology condition (i.e. having no closed timelike curves). Suppose $(M, \mathbf{g})$ admits a $C^{r}$ null asymptote $(\tilde{M}, \tilde{\mathbf{g}})$ such that every null geodesic of $(M, \mathbf{g})$ admits future and past endpoints in $\tilde{M}$. Then $(M, \mathbf{g})$ is a simple space-time and $(\tilde{M}, \tilde{\mathbf{g}})$ is a $C^{r}$ asymptotic null completion of $(M, \mathbf{g})$.

Penrose's definition of an asymptotically simple space-time, in the case of a null conformal boundary, is more restrictive than the preceding definition of a simple space-time in one minor respect. Here the conformal equivalence of the metrics $\tilde{\mathbf{g}} \mid M$ and $\mathbf{g}$ on $M$ is required to satisfy only condition (IV) of Definition 3.1, this being sufficient to guarantee that any simple space-time is null geodesically complete. However Penrose imposes the more stringent requirement that there exists a $C^{1}$ function $\Omega: \tilde{M} \rightarrow \mathbb{R}_{+}$such that $\tilde{\mathbf{g}} \mid M=\Omega^{2} \mathbf{g}$, with $\Omega \mid \partial \tilde{M}=0$ and $\nabla \Omega \mid \partial \tilde{M} \neq 0$. In doing so he is able to obtain important results concerning asymptotic geometric structure, a topic not considered in this paper.

A theorem of Geroch [6] would appear to adapt to the present situation to give that any simple space-time $(M, \mathbf{g})$ must admit a unique asymptotic null completion which extends every other asymptotic null completion of $(M, \mathbf{g})$. (Every asymptotic null completion automatically satisfies his regularity condition.) Unfortunately Geroch's argument is incorrect since it does not properly take account of the fact that, if two future (respectively past) endless null geodesics of $(M, \mathbf{g})$ have a common future (past) endpoint in one asymptotic null completion, they may have distinct future (past) endpoints in another. To understand the possible pathologies in more detail, let $\hat{M}$ be the union of all point sets of all underlying manifolds-with-boundary of all $C^{r}$ asymptotic null completions of $(M, \mathbf{g})$ and, following Geroch [6], for any two asymptotic null completions $\left(\tilde{M}^{\prime}, \tilde{\mathbf{g}}^{\prime}\right)$ and $\left(\tilde{M}^{\prime \prime}, \tilde{\mathbf{g}}^{\prime \prime}\right)$ of $(M, \mathbf{g})$ and any points $p^{\prime} \in \tilde{M}^{\prime}, p^{\prime \prime} \in \tilde{M}^{\prime \prime}$, write $p^{\prime} \sim p^{\prime \prime}$ iff the null geodesics of $(M, \mathbf{g})$ which have future (respectively past) endpoints at $p^{\prime}$ in $\tilde{M}^{\prime}$ are the same as those which have future (past) endpoints at $p^{\prime \prime}$ in $\tilde{M}^{\prime \prime}$. Let $\pi: \hat{M} \rightarrow \hat{M} / \sim=: \tilde{M}_{\infty}$ be the natural projection. Clearly $\tilde{M}_{\infty}$ inherits a projective topology with respect to which it is paracompact. However $\tilde{M}_{\infty}$ needed not be Hausdorff. For if $\mu$ and $v$ are two null geodesics having a common future endpoint $p_{1} \in \partial \tilde{M}_{1}$ in one asymptotic null completion $\left(\tilde{M}_{1}, \tilde{\mathbf{g}}_{1}\right)$, and distinct future endpoints $p_{2 \mu}, p_{2 v} \in \partial \tilde{M}_{2}$ in another $\left(\tilde{M}_{2}, \tilde{\mathbf{g}}_{2}\right)$, then the distinct points $\pi\left(p_{1}\right)$ and $\pi\left(p_{2 \mu}\right)$ of $\tilde{M}_{\infty}$ are both endpoints of $\mu$ in $\widetilde{M}_{\infty}$. Moreover $\tilde{M}_{\infty}$ need not admit the structure of a topological manifold-withboundary. For if $\left(\tilde{M}_{1}, \tilde{\mathbf{g}}_{1}\right)$ and $\left(\tilde{M}_{2}, \tilde{\mathbf{g}}_{2}\right)$ are the only two asymptotic null completions 
of $(M, \mathbf{g})$, and there is just one pair of points $q_{1} \in \partial \tilde{M}_{1}, q_{2} \in \partial \tilde{M}_{2}$ such that $q_{1} \sim q_{2}$, then $\widetilde{M}_{\infty}$ may be obtained by the identification of $\left(\tilde{M}_{1}\right)^{\circ}$ with $\left(\tilde{M}_{2}\right)^{\circ}$ and of $q_{1} \in \partial \tilde{M}_{1}$ with $q_{2} \in \partial \tilde{M}_{2}$. Thus there can be no result that $\tilde{M}_{\infty}$ underlines a unique maximal asymptotic null completion of $(M, \mathbf{g})$. Presumably such a result would hold for a generalization of Definition 4.1 which required that $\tilde{M}$ need be no more than a paracompact topological space. But a definition of this type would be of little use. The present definition will therefore be retained, along with the associated possibility of non-uniqueness.

Henceforth $(M, \mathbf{g})$ will denote any simple space-time, and $(\tilde{M}, \tilde{\mathbf{g}})$ a $C^{r}$ asymptotic null completion of $(M, \mathbf{g})$ for some fixed $r \geqq 0$. The following result is basic.

Lemma 4.2. Let $\mu$ be a future endless causal curve of $(\tilde{M}, \tilde{\mathbf{g}})$ other than a generating segment of $\mathscr{I}^{-}$. Then $M \subset I^{-}(|\mu|, \tilde{M})$.

Proof. Since $\mu$ cuts $M \cup \mathscr{I}^{+}$the set $I^{-}(|\mu|, \tilde{M})$ has non-empty intersection with $M$. Since $I^{-}(|\mu|, \tilde{M})$ does not intersect $\mathscr{I}^{+}$, the set $\dot{I}^{-}(|\mu|, \tilde{M})$ is non-empty. Let $p \in \dot{I}^{-}(|\mu|, \tilde{M})$ and let $\gamma$ be a null geodesic generator of $\dot{I}^{-}(|\mu|, \tilde{M})$ through $p$. Then $\gamma$ either has a future endpoint at some $q \in \overline{|\mu|}$ or is future endless in $\tilde{M}$.

Suppose $\gamma$ has a future endpoint $q \in \overline{|\mu|}$. Let $\gamma^{\prime}$ be the segment of $\gamma$ from $p$ to $q$. Let $q_{i} \in|\mu|$ be a sequence converging to $q$. For each $i$ let $v_{i}$ be a future endless segment of $\mu$ from $q_{i}$. Let $v$ be a future endless causal cluster curve of the $v_{i}$ from $q$. One has $|v| \subset \overline{|\mu|}$. The concatenation of $\gamma^{\prime}$ and $v$ is a future endless causal curve $\sigma$ from $p$ through $q$. If $\sigma$ was not a null geodesic there would exist $r \in|v| \cap I^{+}(p, \tilde{M})$ and hence $r^{\prime} \in|\mu| \cap I^{+}(p, \tilde{M})$, and one would have $p \in I^{-}(|\mu|, \tilde{M})$ giving a contradiction. If $\sigma$ is a null geodesic then, being future endless, it is a generating segment of either $\mathscr{I}^{+}$or $\mathscr{I}^{-}$. In this case one has $p \in \mathscr{I}^{+} \cup \mathscr{I}^{-}=\partial \tilde{M}$.

Suppose $\gamma$ is future endless in $\tilde{M}$. Being a null geodesic, $\gamma$ is a generating segment of either $\mathscr{I}^{+}$or $\mathscr{I}^{-}$and one again has $p \in \partial \tilde{M}$.

One now has $\dot{I}^{-}(|\mu|, \tilde{M}) \subset \partial \tilde{M}$. Since $I^{-}(|\mu|, \tilde{M}) \cap M$ is non-empty there follows $M \subset I^{-}(|\mu|, \tilde{M})$.

Although Definition 4.1 requires that $(M, \mathbf{g})$ satisfies only the chronology condition, the following result shows that strong causality must always hold.

Proposition 4.3. $(M, \mathbf{g})$ is strongly causal.

Proof. Since $(M, \mathbf{g})$ satisfies the chronology condition, and no closed timelike curve of $(\tilde{M}, \tilde{\mathbf{g}})$ could cut $\partial \tilde{M},(\tilde{M}, \tilde{\mathbf{g}})$ must satisfy the chronology condition. If $(\tilde{M}, \tilde{\mathbf{g}})$ violated the causality condition at some point $p \in M$, there would exist an endless, closed null geodesic of $(\tilde{M}, \tilde{\mathbf{g}})$ through $p$. This is impossible since every null geodesic of $(\tilde{M}, \tilde{\mathbf{g}})$ which cuts $M$ must have both future and past endpoints in $\tilde{M}$. Thus $(\tilde{M}, \tilde{\mathbf{g}})$ satisfies the causality condition at every point of $M$.

Suppose $(M, \mathbf{g})$ violates the strong causality condition at some point $q \in M$. Let $r \in I^{+}(q, M)$. There then exists a neighbourhood $\mathscr{N}$ of $q$ in $M$ such that, for every neighbourhood $\mathscr{N}^{\prime} \subset \mathscr{N}$ of $q$, there exists a causal curve $\lambda_{i}$ of $(M, \mathbf{g})$ from $\mathscr{N}^{\prime}$ to $\mathscr{N}^{\prime}$ which cuts $M-\mathscr{N}$. Let $\mathcal{O}_{i} \subset \mathcal{N} \cap I^{-}(r, M)$ be a decreasing sequence of neighbourhoods of $q$ such that $\bigcap_{i} \mathcal{O}_{i}=\{q\}$. For each $i$ there exists, from some $q_{i}^{-} \in \mathcal{O}_{i}$ to some $q_{i}^{+} \in \mathcal{O}_{i}$, a causal curve $\lambda_{i}$ of $(M, \mathbf{g})$ which cuts $M-\mathscr{N}$. From $q$ there is a 
causal cluster curve $\lambda$ of the $\lambda_{i}$ in $\tilde{M}$ having either a future endpoint at $q$ or no future endpoint in $\tilde{M}$. The former case gives rise to a contradiction because $(\tilde{M}, \tilde{\mathbf{g}})$ satisfies the causality condition at $q \in M$. Thus $\lambda$ is future endless in $\tilde{M}$. By Lemma 4.2 one therefore has $M \subset I^{-}(|\lambda|, \tilde{M})$ and hence that there exists $s \in|\lambda| \cap I^{+}(r, \tilde{M})$. Choose $j>0$ such that there exists $s_{j} \in\left|\lambda_{j}\right| \cap I^{+}(r, \tilde{M})$. Since $\lambda_{j}$ has a future endpoint at $q_{j}^{+} \in \mathcal{O}_{j} \subset I^{-}(r, \tilde{M})$, it follows that $(\tilde{M}, \tilde{\mathbf{g}})$ violates the chronology condition at $r \in M$ and one again has a contradiction. Thus $(M, \mathbf{g})$ is strongly causal.

Since $(M, \mathbf{g})$ is strongly causal, one has that $(\tilde{M}, \tilde{\mathbf{g}})$ satisfies the strong causality condition at every point of $M$. However this does not prevent $(\tilde{M}, \tilde{\mathbf{g}})$ from violating strong causality at points of $\mathscr{I}^{+}$and $\mathscr{I}^{-}$. The next result gives a necessary and sufficient condition for strong causality violation at a point of $\mathscr{I}^{+}$. A stronger result will be obtained later.

Lemma 4.4. Let $q \in \mathscr{I}^{+}$. Then strong causality is violated at $q$ iff $M \subset I^{-}\left(\mathcal{O}_{q}, \tilde{M}\right)$ for every neighbourhood $\mathcal{O}_{q}$ of $q$ in $\tilde{M}$.

Proof. Suppose strong causality is violated at $q$. Then there exists a neighbourhood $\mathscr{N}$ of $q$ such that, for every neighbourhood $\mathscr{N}^{\prime} \subset \mathcal{N}$ of $q$, there exists a causal curve from $\mathscr{N}^{\prime}$ to $\mathscr{N}^{\prime}$ which cuts $\tilde{M}-\mathscr{N}$. Let $\mathcal{O}_{q}$ be a neighbourhood of $q$. Let $\mathcal{O}_{i} \subset \mathscr{N} \cap \mathcal{O}_{q}$ be a decreasing sequence of neighbourhoods of $q$ such that $\bigcap_{i} \mathcal{O}_{i}=\{q\}$.

For each $i$ there exists a causal curve $\mu_{i}$ of $(\tilde{M}, \tilde{\mathbf{g}})$ from $\mathcal{O}_{i}$ to $\mathcal{O}_{i}$ which cuts $\tilde{M}-\mathscr{N}$. The $\mu_{i}$ admit a non-degenerate causal cluster curve $\mu$ from $q$ which is a generating segment of $\mathscr{I}^{+}$having either a future endpoint at $q$ or no future endpoint in $\tilde{M}$. In the former case the future endless generating segment of $\mathscr{I}^{+}$from $q$ is a concatenation $\mu^{\infty}$ of a sequence of copies of $\mu$, and Lemma 4.2 gives $M \subset I^{-}\left(\left|\mu^{\infty}\right|, \tilde{M}\right) \subset I^{-}(q, \tilde{M}) \subset I^{-}\left(\mathcal{O}_{q}, \tilde{M}\right)$. Suppose $\mu$ is future endless in $\tilde{M}$. Then Lemma 4.2 gives $M \subset I^{-}(|\mu|, \tilde{M})$ and for any $p \in M$ there exists $r \in|\mu| \cap I^{+}(p, \tilde{M})$. The set $I^{+}(p, \tilde{M})$ is a neighbourhood of $r \in|\mu|$ in $\tilde{M}$ and so is cut by some $\mu_{j}$. Since this $\mu_{j}$ has a future endpoint in $\mathcal{O}_{j} \subset \mathcal{O}_{q}$ there follows $p \in I^{-}\left(\mathcal{O}_{q}, \tilde{M}\right)$, and one again has $M \subset I^{-}\left(\mathcal{O}_{q}, \tilde{M}\right)$.

Suppose $M \subset I^{-}\left(\mathcal{O}_{q}, \tilde{M}\right)$ for every neighbourhood $\mathcal{O}_{q}$ of $q$. Let $\mathcal{O}_{i}$ be a decreasing sequence of compact neighbourhoods of $q$ such that $\bigcap_{i} \mathcal{O}_{i}=\{q\}$. For each $i$ one has $M \subset I^{-}\left(\mathcal{O}_{i}, \tilde{M}\right)$ so by Lemma 3.6 there exists a point $q_{i} \in \mathcal{O}_{i}$ at which strong causality is violated. Since the $q_{i}$ converge to $q$, and the strong causality violating set of $\tilde{M}$ is closed, it follows that strong causality is violated at $q$.

The set of points of $\mathscr{I}^{+}$at which strong causality holds is henceforth denoted by $\mathscr{I}_{0}^{+}$. Since the strongly causal region of $\tilde{M}$ is open, $\mathscr{I}_{0}^{+}$is a relative open submanifold of $\mathscr{I}^{+}$.

Lemma 4.5. Let $\mathscr{K} \subset M$ be compact. Then

(I) $J^{+}(\mathscr{K}, \tilde{M})$ is closed in $\tilde{M}$;

(II) $j^{+}(\mathscr{K}, \tilde{M})$ is compact;

(III) $\dot{J}^{+}(\mathscr{K}, \tilde{M}) \cap \mathscr{I}^{+}$is a compact, acausal subset of $\mathscr{I}_{0}^{+}$ 
Proof.

(I) Suppose there exists $r \in \bar{J}^{+}(\mathscr{K}, \tilde{M})-J^{+}(\mathscr{K}, \tilde{M})$. Then there exists a past endless null geodesic generating segment $\mu$ of $j^{+}(\mathscr{K}, \tilde{M})$ to $r$. The null geodesic $\mu$, being past endless in $\tilde{M}$, is a generating segment of either $\mathscr{I}^{+}$or $\mathscr{I}^{-}$.

Suppose $\mu$ is a generating segment of $\mathscr{I}^{-}$. Then Lemma 4.2 gives $\mathscr{K} \subset I^{+}(|\mu|, \tilde{M})$. Let $\mathscr{N} \subset M$ be a compact neighbourhood of $\mathscr{K}$. Choose $q_{1} \in \mathscr{K}$ and $p_{1} \in|\mu| \cap$ $I^{-}\left(q_{1}, \tilde{M}\right) \subset \mathscr{I}^{-}$. Let $p_{1}^{+} \in I^{-}\left(q_{1}, \tilde{M}\right) \cap I^{+}\left(p_{1}, \tilde{M}\right)-\mathscr{N}$. Since $p_{1}$ is a point of $|\mu| \subset \dot{J}^{+}(\mathscr{K}, \tilde{M})$ one has $p_{1}^{+} \in I^{+}(\mathscr{K}, \tilde{M})$, so there exists $q_{2} \in \mathscr{K} \cap I^{-}\left(p_{1}^{+}, \tilde{M}\right)$. Let $\lambda_{1}$ be a past-directed timelike curve from $q_{1} \in \mathscr{K}$ through $p_{1}^{+} \in \tilde{M}-\mathscr{N}$ to $q_{2} \in \mathscr{K}$. Continue inductively to define a sequence $q_{i} \in \mathscr{K} \subset \mathscr{N}$ such that for each $i$ there exists a past-directed timelike curve $\lambda_{i}$ from $q_{i}$ to $q_{i+1}$ which cuts $\tilde{M}-\mathscr{N}$. The concatenation of the $\lambda_{i}$ is a past-directed past endless timelike curve $\lambda$ from $q_{1}$. Since $\mathscr{K}$ is compact, the $q_{i} \in \mathscr{K}$ admit a cluster point $q \in \mathscr{K}$. One must have $q \in L^{+}(\mu, \tilde{M})$. But this is impossible since strong causality holds at $q \in \mathscr{K} \subset M$. It follows that $\mu$ is a generating segment of $\mathscr{I}^{+}$.

One now has $r \in \mathscr{I}^{+}$and more generally $\bar{J}^{+}(\mathscr{K}, \tilde{M})-J^{+}(\mathscr{K}, \tilde{M}) \subset \mathscr{I}^{+}$. Thus $J^{+}(\mathscr{K}, \tilde{M}) \cup \mathscr{I}^{+}$is closed in $\tilde{M}$. Let $\mathscr{N}_{r} \subset \tilde{M}-\mathscr{K}$ be a connected open neighbourhood of $r$ in $\tilde{M}$. There exists $r^{\prime} \in J^{+}(\mathscr{K}, \tilde{M}) \cap \mathscr{N}_{r}$. Since $\mathscr{K}$ does not intersect $\mathscr{N}_{r} \cup \mathscr{I}^{+}$there exists a non-degenerate causal curve from $\mathscr{K}$ to $r^{\prime}$ which does not cut $\mathscr{I}^{+}$, except possibly at $r^{\prime}$. Hence one cannot have $\mathscr{N}_{r}-\mathscr{I}^{+} \subset \tilde{M}-J^{+}(\mathscr{K}, \tilde{M})$. But neither can one have $\mathscr{N}_{r}-\mathscr{I}^{+} \subset J^{+}(\mathscr{K}, \tilde{M})$, for then $r$ would be an interior point of $J^{+}(\mathscr{K}, \tilde{M})$. The set $\mathscr{N}_{r}-\mathscr{I}^{+}$, being connected, therefore has non-empty intersection with $j^{+}(\mathscr{K}, \tilde{M})$. Let $\mathscr{N}_{i} \subset \tilde{M}-\mathscr{K}$ be a decreasing sequence of connected open neighbourhoods of $r$ in $\tilde{M}$ such that $\bigcap_{i} \mathscr{N}_{i}=\{r\}$. For each $i$ there exists $r_{i} \in\left(\mathscr{N}_{i}-\mathscr{I}^{+}\right) \cap \dot{J}^{+}(\mathscr{K}, \tilde{M})$ and a null geodesic generating segment $v_{i}$ of $\dot{J}^{+}(\mathscr{K}, \tilde{M})$ from $\mathscr{K}$ to $r_{i}$. Since the $r_{i}$ converge to $r$, the $v_{i}$ admit a cluster curve $v$ which is a null geodesic generating segment of $\dot{J}^{+}(\mathscr{K}, \tilde{M})$ from $\mathscr{K}$ having either a future endpoint at $r$ or no future endpoint in $\tilde{M}$. In the latter case $v$ would have to be a generating segment of $\mathscr{I}^{+}$or $\mathscr{I}^{-}$, and this is impossible since $v$ has a past endpoint in $\mathscr{K} \subset M$. Hence $v$ has a future endpoint at $r$ and one has $r \in J^{+}(\mathscr{K}, \tilde{M})$ contrary to hypothesis.

It now follows that $\bar{J}^{+}(\mathscr{K}, \tilde{M})-J^{+}(\mathscr{K}, \tilde{M})$ is empty, and hence that $J^{+}(\mathscr{K}, \tilde{M})$ is closed.

(II) Suppose $j^{+}(\mathscr{K}, \tilde{M})$ is non-compact. Let $t_{i}$ be a sequence of points therein without cluster point in $\tilde{M}$. Since $J^{+}(\mathscr{K}, \tilde{M})$ is closed there exists, for each $i$, a generating segment $\lambda_{i}$ of $\dot{J}^{+}(\mathscr{K}, \tilde{M})$ from some $s_{i} \in \mathscr{K}$ to $t_{i}$. Since $\mathscr{K}$ is compact, the $s_{i}$ admit a cluster point $s \in \mathscr{K}$. Hence the $\lambda_{i}$ admit a cluster curve $\lambda$ which is a generator of $\dot{J}^{+}(\mathscr{K}, \tilde{M})$ having a past endpoint at $s \in \mathscr{K}$ and no future endpoint in $\tilde{M}$. But then $\lambda$ should be a generating segment of either $\mathscr{I}^{+}$or $\mathscr{I}^{-}$, which is impossible since $\lambda$ has a past endpoint at $s \in \mathscr{K} \subset M$. The set $\dot{J}^{+}(\mathscr{K}, \tilde{M})$ is therefore compact.

(III) Suppose $\Sigma:=\dot{J}^{+}(\mathscr{K}, \tilde{M}) \cap \mathscr{I}^{+}$is not acausal. Then there exists a nondegenerate null geodesic generating segment $\sigma^{+}$of $\mathscr{I}^{+}$from some $u \in \dot{J}^{+}(\mathscr{K}, \tilde{M}) \subset$ $J^{+}(\mathscr{K}, \tilde{M})$ to some $w \in J^{+}(\mathscr{K}, \tilde{M})$. If some $v \in\left|\sigma^{+}\right| \subset J^{+}(\mathscr{K}, \tilde{M})$ was an interior point of $J^{+}(\mathscr{K}, \tilde{M})$ there would exist $v^{\prime} \in I^{-}(v, \tilde{M}) \cap J^{+}(\mathscr{K}, \tilde{M})$. But this would imply $w \in I^{+}(\mathscr{K}, \tilde{M})$ which contradicts $w \in \dot{J}^{+}(\mathscr{K}, \tilde{M})=\dot{I}^{+}(\mathscr{K}, \tilde{M})$. It follows that $\sigma^{+}$is a 
generating segment of $\dot{J}^{+}(\mathscr{K}, \tilde{M})$. Let $\sigma^{-}$be a causal curve from $\mathscr{K}$ to $u$. The concatenation of $\sigma^{-}$and $\sigma^{+}$is a causal curve $\sigma$ from $\mathscr{K}$ to $w$. Since $\sigma^{+}$is a non-degenerate generating segment of $\mathscr{I}^{+}$, and the past endpoint of $\sigma^{-}$lies in $\tilde{M}-\mathscr{I}^{+}, \sigma$ cannot be a null geodesic. Hence there exists a timelike curve from $\mathscr{K}$ to $w$. But this implies $w \in I^{+}(\mathscr{K}, \tilde{M})$ so one again has a contradiction. Thus $\Sigma$ is acausal.

Suppose strong causality is violated at some point $y \in \Sigma$. By Lemma 4.4 one has $M \subset I^{-}(\mathcal{N}, \tilde{M})$ for every neighbourhood $\mathscr{N}$ of $y$. Let $x \in I^{+}(\mathscr{K}, \tilde{M}) \cap M$. Then there exists a sequence of points $y_{i}$ converging to $y$ such that, for each $i$, there exists a timelike curve $\lambda_{i}$ from $x$ to $y_{i}$. The $\lambda_{i}$ admit a causal cluster curve $\lambda$ to $y$ which is either past endless in $\tilde{M}$ or has a past endpoint at $x$. If $\lambda$ had a past endpoint at $x$ one would have $y \in I^{+}(\mathscr{K}, \tilde{M})$ which is incompatible with $y \in \Sigma \subset \dot{J}^{+}(\mathscr{K}, \tilde{M})=\dot{I}^{+}(\mathscr{K}, \tilde{M})$. Thus $\lambda$ is past endless in $\tilde{M}$. The closed set $J^{+}(\mathscr{K}, \tilde{M})$ contains $\left|\lambda_{i}\right|$ for each $i$, and so contains $|\lambda|$. Moreover $\lambda$, having a future endpoint at $y \in \dot{J}^{+}(\mathscr{K}, \tilde{M})$, cannot cut the interior of $J^{+}(\mathscr{K}, \tilde{M})$. It follows that $\lambda$ is a null geodesic generator of $\dot{J}^{+}(\mathscr{K}, \tilde{M})$. But $\lambda$ is past endless in $\tilde{M}$ with a future endpoint at $y \in \mathscr{I}^{+}$, and so must be a generating segment of $\mathscr{I}^{+}$. One thus has $|\lambda| \subset \dot{J}^{+}(\mathscr{K}, \tilde{M}) \cap$ $\mathscr{I}^{+}=\Sigma$. This is impossible since $\Sigma$ is acausal. Hence $\Sigma \subset \mathscr{I}_{0}^{+}$.

If $\mathscr{K}$ is a compact set of $M$, then $J^{+}(\mathscr{K}, \tilde{M})$ is a future set of $(\tilde{M}, \tilde{\mathbf{g}})$, and it follows by Lemmas 3.4 and 4.5 that $\dot{J}^{+}(\mathscr{K}, \tilde{M})$ is a compact achronal embedded topological 3-submanifold-with-boundary of $\tilde{M}$ such that $\partial\left(\dot{J}^{+}(\mathscr{K}, \tilde{M})\right)=\dot{J}^{+}(\mathscr{K}, \tilde{M}) \cap \mathscr{I}^{+}$.

The use of Lemma 4.5 facilitates a generalization of Lemma 4.4.

Proposition 4.6 For any $q \in \mathscr{I}^{+}$the following conditions are equivalent:

(I) strong causality is violated at q;

(II) $M \subset I^{-}(\mathcal{N}, \tilde{M})$ for every neighbourhood $\mathscr{N}$ of $q$ in $\tilde{M}$;

(III) $M \subset I^{-}(q, \tilde{M})$.

Proof. Since (III) implies (II) which, by Lemma 4.4, implies (I), it suffices to show that (I) implies (III).

Suppose strong causality is violated at $q \in \mathscr{I}^{+}$, and suppose there exists $p \in M-I^{-}(q, \tilde{M})$. Let $p^{+} \in I^{+}(p, \tilde{M}) \cap M$. One cannot have $q \in J^{+}\left(p^{+}, \tilde{M}\right)$ for this would imply $p \in I^{-}(q, \tilde{M})$ which is contrary to hypothesis. Hence $q \in \tilde{M}-J^{+}\left(p^{+}, \tilde{M}\right)$. Since Lemma 4.5 gives that $J^{+}\left(p^{+}, \tilde{M}\right)$ is closed, the point $q \in \mathscr{I}^{+}$therefore admits an open neighbourhood $\mathscr{N}_{q}$ which does not intersect $J^{+}\left(p^{+}, \tilde{M}\right)$. There follows $p^{+} \in M-I^{-}\left(\mathscr{N}_{q}, \tilde{M}\right)$ which is impossible since Lemma 4.4 gives $M \subset I^{-}\left(\mathscr{N}_{q}, \tilde{M}\right)$. Hence if strong causality is violated at $q \in \mathscr{I}^{+}$then $M \subset I^{-}(q, \tilde{M})$.

Corollary. Let $\mu$ be an endless null geodesic of $(M, \mathbf{g})$ and let $p \in \mathscr{I}^{+}$be the future endpoint of $\mu$ in $\tilde{M}$. Then strong causality is violated at $p$ iff $M \subset I^{-}(|\mu|, M)$.

Proof. Strong causality is violated at $p$ iff $M \subset I^{-}(p, \tilde{M}) \cap M=I^{-}(|\mu| \tilde{M}) \cap M=$ $I^{-}(|\mu|, M)$.

This corollary relates the causal structure at $\mathscr{I}^{+}$to intrinsic properties of $(M, \mathbf{g})$. It shows that strong causality holds at $\mathscr{I}^{+}$iff no null geodesic of $(M, \mathbf{g})$ 
cuts the causal future of every point of $M$. Similarly strong causality holds at $\mathscr{I}^{-}$ iff no null geodesic of $(M, \mathbf{g})$ cuts the causal past of every point of $M$. These observations give rise to the physical interpretations offered in the Introduction for strong causality violation at $\mathscr{I}^{+}$and $\mathscr{I}^{-}$.

Another consequence of the corollary to Proposition 4.6 is that, if $(M, \mathbf{g})$ admits an asymptotic null completion which is strongly causal at $\mathscr{I}^{+}$, then every asymptotic null completion of $(M, \mathbf{g})$ is strongly causal at $\mathscr{I}^{+}$. On the other hand, if $(M, \mathbf{g})$ admits an asymptotic null completion which violates strong causality at $\mathscr{I}^{+}$, then every asymptotic null completion of $(M, \mathbf{g})$ must violate strong causality at $\mathscr{I}^{+}$.

The next result is of central importance.

Proposition 4.7. $(M, \mathrm{~g})$ is globally hyperbolic.

Proof. Suppose there exist points $p, r \in M$ such that $J^{+}(p, M) \cap J^{-}(r, M)$ is a noncompact set of $M$. Then there exists a sequence $q_{i} \in J^{+}(p, M) \cap J^{-}(r, M)$ without cluster point in $J^{+}(p, M) \cap J^{-}(r, M)$. The $q_{i}$ are contained in the set $J^{+}(p, \tilde{M}) \cap$ $J^{-}(r, \tilde{M}) \subset M$ which, by Lemma 4.5 , is closed in $\tilde{M}$. The $q_{i}$ are therefore without cluster point in $\tilde{M}$. For each $i$ let $\mu_{i}$ be a causal curve of $(\tilde{M}, \tilde{\mathbf{g}})$ from $p$ to $q_{i}$. The $\mu_{i}$ admit a future endless causal cluster curve $\mu$ in $(\tilde{M}, \tilde{\mathbf{g}})$ from $p$. The closed set $J^{-}(r, \tilde{M})$ of $\tilde{M}$ contains $\left|\mu_{i}\right|$ for all $i$ and therefore contains $|\mu|$. Hence by Lemma 4.2 one has $r \in M \subset I^{-}(|\mu|, \tilde{M}) \subset J^{-}(r, \tilde{M})$. This gives a contradiction since $(\tilde{M}, \tilde{\mathbf{g}})$ satisfies the strong causality condition at $r \in M$. Hence $J^{+}(p, M) \cap J^{-}(r, M)$ is compact for all $p, r \in M$, and $(M, \mathbf{g})$, being strongly causal, is globally hyperbolic.

It follows that the space-time $(M, \mathbf{g})$ admits Cauchy surfaces. The next result identifies the basic properties of an arbitrary Cauchy surface $\mathscr{C}$ of $(M, \mathbf{g})$ in relation to $(\tilde{M}, \tilde{\mathbf{g}})$.

Proposition 4.8. Let $\mathscr{C}$ be a Cauchy surface of $(M, \mathbf{g})$. Then

(I) $\mathscr{C}$ is closed and acausal in $(\tilde{M}, \tilde{\mathbf{g}})$;

(II) $\mathscr{I}^{+} \subset I^{+}(\mathscr{C}, \tilde{M}), \mathscr{I}^{-} \subset \tilde{M}-J^{+}(\mathscr{C}, \tilde{M})$;

(III) $J^{+}(\mathscr{C}, \tilde{M})$ is closed in $\tilde{M}$.

Proof.

(I) A non-degenerate causal curve of $(\tilde{M}, \tilde{\mathbf{g}})$ from $\mathscr{C} \subset M$ to $\mathscr{C} \subset M$ could not cut $\mathscr{I}^{+}$or $\mathscr{I}^{-}$. Any such curve would therefore be a non-degenerate causal curve of $(M, \mathbf{g})$ from $\mathscr{C}$ to $\mathscr{C}$. Since $\mathscr{C}$ is acausal in $(M, \mathbf{g})$ it follows that $\mathscr{C}$ is acausal in $(\tilde{M}, \tilde{\mathbf{g}})$.

Suppose there exists $r \in \tilde{M}-\mathscr{C}$ such that every neighbourhood of $r$ in $\tilde{M}$ intersects $\mathscr{C}$. Since $\mathscr{C}$ is relatively closed in $M$ one must have $r \in \mathscr{I}^{+} \cup \mathscr{I}^{-}$. Suppose $r \in \mathscr{I}^{+}$. There exists a null geodesic $v^{\prime}$ of $(\tilde{M}, \tilde{\mathbf{g}})$ from $\mathscr{I}^{-}$to $r$ and this may be deformed to a timelike curve $v$ of $(\tilde{M}, \tilde{\mathbf{g}})$ from $\mathscr{I}^{-}$to $r$. The maximal segment of $v$ in $M$ is an endless timelike curve of $(M, \mathbf{g})$ cutting $\mathscr{C}$ at some point $q \in \mathscr{C}$. But then $I^{+}(q, \tilde{M})$ is a neighbourhood of $r$ and must intersect $\mathscr{C}$. This is impossible since $\mathscr{C}$ is acausal in $(\tilde{M}, \tilde{\mathbf{g}})$. A similar contradiction is obtained for $r \in \mathscr{I}^{-}$. Hence $\mathscr{C}$ is closed in $\tilde{M}$.

(II) Let $t \in \mathscr{I}^{+}$. Let $\rho$ be a timelike curve of $(\tilde{M}, \tilde{\mathbf{g}})$ from $\mathscr{I}^{-}$to $t$. The maximal segment of $\rho$ in $M$ is an endless timelike curve of $(M, \mathbf{g})$ cutting $\mathscr{C}$ at some point $s \in \mathscr{C}$. There follows $t \in I^{+}(s, \tilde{M}) \subset I^{+}(\mathscr{C}, \tilde{M})$ and more generally $\mathscr{I}^{+} \subset I^{+}(\mathscr{C}, \tilde{M})$. 
The inclusion $\mathscr{I}^{-} \subset \tilde{M}-J^{+}(\mathscr{C}, \tilde{M})$ is an immediate consequence of $\mathscr{C} \cap \mathscr{I}^{-}=\varnothing$. (III) Suppose there exists $u \in \bar{J}^{+}(\mathscr{C}, \tilde{M})-J^{+}(\mathscr{C}, \tilde{M})$. If $u \in M$ then $u \in M-$ $J^{+}(\mathscr{C}, \tilde{M}) \subset M-J^{+}(\mathscr{C}, M)=I^{-}(\mathscr{C}, M)$, whence $I^{-}(\mathscr{C}, \tilde{M})$ is a neighbourhood of $u$ and must intersect $J^{+}(\mathscr{C}, \tilde{M})$. This contradicts the acausality of $\mathscr{C}$ in $(\tilde{M}, \tilde{\mathbf{g}})$. Since (II) gives $\mathscr{I}^{+} \subset I^{+}(\mathscr{C}, \tilde{M}) \subset J^{+}(\mathscr{C}, \tilde{M})$, it only remains to consider the possibility $u \in \mathscr{I}^{-}$. Let $\lambda$ be a timelike curve of $(\tilde{M}, \tilde{\mathbf{g}})$ from $u \in \mathscr{I}^{-}$to $\mathscr{I}^{+}$. The maximal segment of $\lambda$ in $M$ is an endless timelike curve of $(M, \mathbf{g})$ cutting $\mathscr{C}$ at some point $v \in \mathscr{C}$. But then $I^{-}(v, \tilde{M})$ is a neighbourhood of $u$ and so must intersect $J^{+}(\mathscr{C}, \tilde{M})$. It follows that $I^{-}(v, \tilde{M})$ intersects $\mathscr{C}$ and one again has a contradiction to the acausality of $\mathscr{C}$ in $(\tilde{M}, \tilde{\mathbf{g}})$. Thus $J^{+}(\mathscr{C}, \tilde{M})$ is closed in $\tilde{M}$.

The next result is somewhat out of place in the present context since it is entirely topological in nature. However it is essential to the proofs of some of the subsequent results on causal structure.

Proposition 4.9. $M, \mathscr{C}, \tilde{M}, \mathscr{I}^{+}$and $\mathscr{I}^{-}$are all connected and non-compact.

Proof. $M$ is connected by hypothesis, and is dense in $\tilde{M}$. Therefore $\tilde{M}$ is connected. Since $M$ is homeomorphic to $\mathscr{C} \times \mathbb{R}$ one has that $\mathscr{C}$ is connected and that $M$ is non-compact.

Let $N_{\mathscr{C}}$ be the bundle of all future-directed null directions over $\mathscr{C}$, and let $N_{+}$ be the bundle of all future-directed null directions not tangent to $\mathscr{I}^{+}$over $\mathscr{I}^{+}$. The fibres of $N_{\mathscr{G}}$ are homeomorphic to $\mathbb{S}^{2}$ whilst the fibres of $N_{+}$are homeomorphic to $\mathbb{S}^{2}-\{p t.\} \approx \mathbb{R}^{2}$. The future-directed null geodesics of $(\tilde{M}, \tilde{\mathbf{g}})$ from $\mathscr{C}$ to $\mathscr{I}^{+}$define a homeomorphism of the total space of $N_{\mathscr{C}}=\mathscr{C} \times \mathbb{S}^{2}$ onto the total space of $N_{+}=\mathscr{I}^{+} \simeq \mathbb{R}^{2}$. Thus $\mathscr{C}$ is non-compact. And since $\mathscr{C}$ is connected, $\mathscr{I}^{+}$must be connected. Similarly $\mathscr{I}^{-}$is connected.

Let $\tilde{\mathbf{V}}$ be a timelike vector field on $\tilde{M}$. The integral curves of $\tilde{\mathbf{V}}$ all cut $\mathscr{C}$ and so define a homeomorphism of $\mathscr{I}^{+}$onto a non-empty open submanifold of $\mathscr{C}$. Since $\mathscr{C}$ is connected and non-compact, every non-empty open submanifold of $\mathscr{C}$ is non-compact. Hence $\mathscr{I}^{+}$is non-compact, and similarly $\mathscr{I}^{-}$is non-compact. Since $\mathscr{I}^{+}$and $\mathscr{I}^{-}$are closed in $\tilde{M}$ it follows that $\tilde{M}$ is non-compact.

Remark 4.10. For any compact set $\mathscr{K}$ of $M$ the sets $\dot{J}^{+}(\mathscr{K}, \tilde{M})$ and $\dot{J}^{+}(\mathscr{K}, \tilde{M}) \cap \mathscr{I}^{+}$ in statements (II) and (III) of Lemma 4.5 are non-empty.

Proof. Since the closed set $J^{+}(\mathscr{K}, \tilde{M})$ contains $\mathscr{K}$ and does not intersect $\mathscr{I}^{-}$, the set $\dot{J}^{+}(\mathscr{K}, \tilde{M})$ is non-empty and does not intersect $\mathscr{I}^{-}$.

Suppose $\dot{J}^{+}(\mathscr{K}, \tilde{M})$ does not intersect $\mathscr{I}^{+}$. Since $J^{+}(\mathscr{K}, \tilde{M})$ is a future set of $(\tilde{M}, \tilde{\mathbf{g}})$, it follows by Lemma 3.3 that $\dot{J}^{+}(\mathscr{K}, \tilde{M})$ is a non-empty compact achronal embedded topological 3-submanifold of $(\tilde{M}, \tilde{\mathbf{g}})$. Let $\tilde{\mathbf{V}}$ be a timelike vector field on $\tilde{M}$. The maximal integral curves of $\tilde{\mathbf{V}}$ all cut the Cauchy surface $\mathscr{C}$ of $(M, \mathbf{g})$ and so define a homeomorphism of $\dot{J}^{+}(\mathscr{K}, \tilde{M})$ onto a non-empty compact open submanifold of $\mathscr{C}$. But $\mathscr{C}$ is connected and non-compact, and so all its non-empty open submanifolds are non-compact. One thus has a contradiction. Hence $j^{+}(\mathscr{K}, \tilde{M}) \cap \mathscr{I}^{+}$is non-empty.

Provisional Definition 4.11. A non-empty compact acausal embedded topological 
2-submanifold of $\mathscr{I}_{0}^{+}$is a slice of $\mathscr{I}^{+}$. A slice of $\mathscr{I}^{+}$of the form $\dot{J}^{+}(\mathscr{K}, \tilde{M}) \cap \mathscr{I}^{+}$ for some compact set $\mathscr{K}$ of $M$ is a good slice of $\mathscr{I}^{+}$.

Lemmas 3.3 and 4.4 with Remark 4.10 together establish the existence of slices, and of good slices in particular. Some of the results in the remainder of this section pertain only to good slices. In the next section it is established that all slices of $\mathscr{I}^{+}$are connected. This fact will enable results for good slices to be generalized to apply to all slices.

Lemma 4.12. Let $\Sigma$ be a slice of $\mathscr{I}^{+}$. Then

(I) $J^{-}(\Sigma, \tilde{M})$ is closed in $\tilde{M}$;

(II) $\dot{J}^{-}(\Sigma, \tilde{M})$ is non-empty compact and achronal;

(III) $\dot{J}^{-}(\Sigma, \tilde{M}) \cap \mathscr{I}^{+}=\Sigma$.

Proof. It will be convenient to break (I) into two parts:

(Ia) $\bar{J}^{-}(\Sigma, \tilde{M})-J^{-}(\Sigma, \tilde{M}) \subset \mathscr{I}^{-}$;

(Ib) $\bar{J}^{-}(\Sigma, \tilde{M})-J^{-}(\Sigma, \tilde{M}) \subset \tilde{M}-\mathscr{I}^{-}$.

The approach will be to first prove (Ia) which will then be used to prove (III). These will then be used to prove (Ib). Finally (I) and (III) will be used to prove (II). (Ia) Suppose there exists $p \in \bar{J}^{-}(\Sigma, \tilde{M})-J^{-}(\Sigma, \tilde{M})$. Then there exists a future endless null geodesic generating segment $\mu$ of $\dot{J}^{-}(\Sigma, \tilde{M})$ from $p$. The null geodesic $\mu$, having only one endpoint in $\tilde{M}$, must be a generating segment of $\mathscr{I}^{+}$or $\mathscr{I}^{-}$.

Suppose $\mu$ is a generating segment of $\mathscr{I}^{+}$. Then Lemma 4.2 gives $M \subset I^{-}(|\mu|, \tilde{M})$. Together with $|\mu| \subset \dot{J}^{-}(\Sigma, \tilde{M})$ this implies that, for any $r \in M$, the open set $I^{+}(r, \tilde{M})$ intersects $J^{-}(\Sigma, \tilde{M})$, and hence that $I^{-}(\Sigma, \tilde{M})$ contains $r$. There follows $M \subset I^{-}(\Sigma, \tilde{M})$ and so, by Lemma 3.6, strong causality must be violated at some point of $\Sigma$. This is contrary to the definition of a slice.

One now has that $\mu$ is a future endless generating segment of $\mathscr{I}^{-}$. The past endpoint $p$ of $\mu$ therefore lies in $\mathscr{I}^{-}$. More generally one has $\bar{J}^{-}(\Sigma, \tilde{M})-$ $J^{-}(\Sigma, \tilde{M}) \subset \mathscr{I}^{-}$.

(III) Let $p \in \dot{J}^{-}(\Sigma, \tilde{M}) \cap \mathscr{I}^{+}$and suppose $p \notin \Sigma$. By (Ia) one has $p \in J^{-}(\Sigma, \tilde{M}) \cap \mathscr{I}^{+}=$ $J^{-}\left(\Sigma, \mathscr{I}^{+}\right)$. Since $\Sigma$ is an acausal 2-submanifold of $\mathscr{I}^{+}$, and because $p$ does not lie in $\Sigma$, the set $J^{-}\left(\Sigma, \mathscr{I}^{+}\right)$is a relative neighbourhood of $p$ in $\mathscr{I}^{+}$. But then $J^{-}\left(J^{-}\left(\Sigma, \mathscr{I}^{+}\right), \tilde{M}\right)=J^{-}(\Sigma, \tilde{M})$ is a neighbourhood of $p$ in $\tilde{M}$. This contradicts $p \in \dot{J}^{-}(\Sigma, \tilde{M})$.

(Ib) Suppose there exists $p \in\left(\bar{J}^{-}(\Sigma, \tilde{M})-J^{-}(\Sigma, \tilde{M})\right) \cap \mathscr{I}^{-}$. Let $\mathscr{N}_{p} \subset \tilde{M}-\Sigma$ be a connected open neighbourhood of $p$ in $\tilde{M}$. There exists $p^{\prime} \in J^{-}(\Sigma, \tilde{M}) \cap \mathscr{N}_{p}$. Since $\Sigma$ does not intersect $\mathscr{N}_{p}$ there exists a non-degenerate causal curve from $p^{\prime}$ to $\Sigma$. This may be deformed to a causal curve from $p^{\prime}$ to $\Sigma$ which does not cut $\mathscr{I}^{-}$, except possibly at $p^{\prime}$. Hence one cannot have $\mathscr{N}_{p}-\mathscr{I}^{-} \subset \tilde{M}-J^{-}(\Sigma, \tilde{M})$. And neither can one have $\mathscr{N}_{p}-\mathscr{I}^{-} \subset J^{-}(\Sigma, \tilde{M})$ for then $p$ would be an interior point of $J^{-}(\Sigma, \tilde{M})$. The set $\mathscr{N}_{p}-\mathscr{I}^{-}$, being connected, therefore has non-empty intersection with $\dot{J}^{-}(\Sigma, \tilde{M})$. Let $\mathscr{N}_{i} \subset \tilde{M}-\Sigma$ be a decreasing sequence of connected open neighbourhoods of $p$ in $\tilde{M}$ such that $\bigcap_{i} \mathscr{N}_{i}=\{p\}$. For each $i$ there exists $p_{i} \in\left(\mathscr{N}_{i}-\mathscr{I}^{-}\right) \cap \dot{J}^{-}(\Sigma, \tilde{M})$ and a null geodesic generating segment $v_{i}$ of $\dot{J}^{-}(\Sigma, \tilde{M})$ from $p_{i}$ to $\Sigma$. Since the $p_{i}$ converge to $p$, the $v_{i}$ admit a cluster curve $v$ which is a 
null geodesic generating segment of $\dot{J}^{-}(\Sigma, \tilde{M})$ to $\Sigma$ having either a past endpoint at $p$ or no past endpoint in $\tilde{M}$. In the former case one would have $p \in J^{-}(\Sigma, \tilde{M})$, contrary to hypothesis. In the latter case $v$, having a future endpoint in $\Sigma \subset \mathscr{I}^{+}$, would be a generating segment of $\mathscr{I}^{+}$. Since $\Sigma$ is acausal, it would follow that $|v|-\Sigma$ is a non-empty subset of $\dot{J}^{-}(\Sigma, \tilde{M}) \cap \mathscr{I}^{+}-\Sigma$. This is contrary to (III).

(II) Suppose $\dot{J}^{-}(\Sigma, \tilde{M})$ is non-compact. Let $p_{i}$ be a sequence of points therein, without cluster point in $\tilde{M}$. Since $J^{-}(\Sigma, \tilde{M})$ is closed there exists, for each $i$, a generating segment $\gamma_{i}$ of $\dot{J}^{-}(\Sigma, \tilde{M})$ from $p_{i}$ to some $q_{i} \in \Sigma$. Since $\Sigma$ is compact the $q_{i}$ admit a cluster point $q \in \Sigma$. The $\gamma_{i}$ therefore admit a cluster curve $\gamma$ which is a generator of $\dot{J}^{-}(\Sigma, \tilde{M})$ to $q \in \mathscr{I}^{+}$having no past endpoint in $\tilde{M}$. It follows that $\gamma$ is a past endless generating segment of $\mathscr{I}^{+}$. Hence $|\gamma|-\Sigma$ is a non-empty subset of $\dot{J}^{-}(\Sigma, \tilde{M}) \cap \mathscr{I}^{+}-\Sigma$. One now has a contradiction by (III).

The set $\dot{J}^{-}(\Sigma, \tilde{M})$ is non-empty since, by (III), it contains $\Sigma$.

Proposition 4.13. Let $\gamma$ be a generator of $\mathscr{I}_{0}^{+}$. Then

(I) $\gamma$ is endless in $\tilde{M}$;

(II) $L^{-}(\gamma, \tilde{M})=\varnothing$;

(III) $\gamma$ cuts every good slice of $\mathscr{I}^{+}$.

Proof.

(I) Suppose $\gamma$ admits a past endpoint $p$ in $\tilde{M}$. Then strong causality is violated at $p$. For any $q \in|\gamma|$ one has $M \subset I^{-}(p, \tilde{M}) \subset I^{-}(q, \tilde{M})$ and hence that strong causality is violated at $q$. This contradicts $|\gamma| \subset \mathscr{I}_{0}^{+}$. Hence $\gamma$ is past endless in $\tilde{M}$.

Suppose $\gamma$ admits a future endpoint $s$ in $\tilde{M}$. Then strong causality is violated at $s$ and one has $M \subset I^{-}(s, \tilde{M})$. Let $t \in J^{+}(s, \tilde{M})-\{s\} \subset \mathscr{I}^{+}$. Then $M \subset I^{-}(s, \tilde{M}) \subset$ $I^{-}(t, \tilde{M})$ and strong causality is violated at $t$. Let $\mathscr{N}_{t}$ be a neighbourhood of $t$ not containing $s$, and let $t_{i} \in \mathscr{N}_{t} \cap M$ converge to $t$ in $\tilde{M}$. For each $i$ there exists a timelike curve $\lambda_{i}$ from $t_{i}$ to $s$. The $\lambda_{i}$ admit a causal cluster curve $\lambda$ to $s$ which either has a past endpoint at $t \in \mathscr{I}^{+}-\mathscr{I}_{0}^{+}$or is past endless in $\tilde{M}$. In the former case one would have $t \in|\lambda|-\{s\} \subset|\gamma| \subset \mathscr{I}_{0}^{+}$which gives a contradiction. Thus $\lambda$ is past endless in $\tilde{M}$. Let $\mathscr{N}_{s}$ be an open neighbourhood of $s$ not intersecting $\mathscr{N}_{t} \cup \mathscr{I}^{-}$. Let $r \in(|\lambda|-\{s\}) \cap \mathscr{N}_{s} \subset|\gamma| \cup M \subset \mathscr{I}_{0}^{+} \cup M$. Then strong causality holds at $r$. Let $\mathscr{N}_{r}^{\prime} \subset \mathscr{N}_{s}$ be a neighbourhood of $r$ in $\tilde{M}$. For any $r^{-} \in I^{-}\left(r, \mathscr{N}_{r}^{\prime}\right)$ one has $t \in I^{+}\left(r^{-}, \tilde{M}\right)$. Hence $I^{+}\left(\mathscr{N}_{r}^{\prime}, \tilde{M}\right)$ is a neighbourhood of $t$ in $\tilde{M}$ and there exists $I>0$ such that $t_{i} \in I^{+}\left(\mathscr{N}_{r}^{\prime}, \tilde{M}\right)$ for all $i>I$. Choose $j>I$ such that $\lambda_{j}$ cuts $\mathscr{N}_{r}^{\prime}$. Then one may join a timelike curve from $\mathscr{N}_{r}^{\prime}$ to $t_{j}$ to a segment of $\lambda_{j}$ from $t_{j}$ to $\mathscr{N}_{r}^{\prime}$ to obtain a causal curve from $\mathscr{N}_{r}^{\prime} \subset \mathcal{N}_{s}$ through $t_{j} \in \mathscr{N}_{t} \subset \tilde{M}-\mathscr{N}_{s}$ to $\mathscr{N}_{r}^{\prime} \subset \mathscr{N}_{s}$. It follows that strong causality is violated at $r$ and one has a contradiction. Thus $\gamma$ is future endless in $\tilde{M}$.

(II) Let $r \in|\gamma|$. Since strong causality holds at $r$ one has $M \not \subset I^{-}(r, \tilde{M})$. And since $I^{-}(r, \tilde{M}) \cap M$ is non-empty there exists $q \in \dot{I}^{-}(r, \tilde{M}) \cap M$. The null geodesic generator of $\dot{I}^{-}(r, \tilde{M})$ through $q \in M$ cannot be future endless in $\tilde{M}$, and so must have a future endpoint at $r$. There follows $r \in J^{+}(q, \tilde{M})$ and hence $r \in \bar{I}^{+}(q, \tilde{M})$ by Lemma 3.3. One cannot have $r \in I^{+}(q, \tilde{M})$, since this would imply $q \in I^{-}(r, \tilde{M})$ which is incompatible with $q \in \dot{I}^{-}(r, \tilde{M})$. Hence $r$ is a point of the good slice $\Sigma:=\dot{I}^{+}(q, \tilde{M}) \cap \mathscr{I}^{+}$of $\mathscr{I}^{+}$. Let $\gamma_{r}$ be the past endless segment of $\gamma$ to $r$. 
Suppose there exists $p \in L^{-}(\gamma, \tilde{M})=L^{-}\left(\gamma_{r}, \tilde{M}\right)$. Then strong causality is violated at $p$. Since $\left|\gamma_{r}\right|$ is contained in the closed set $J^{-}(\Sigma, \tilde{M})$ one has $p \in \overline{\left|\gamma_{r}\right|} \subset J^{-}(\Sigma, \tilde{M})$. Hence there exists a generating segment of $\mathscr{I}^{+}$from $p$ to some $r^{\prime} \in \Sigma$. But strong causality holds at $r^{\prime}$ and so should also hold at $p$ by (I). This establishes a contradiction.

(III) Suppose there exists a compact set $\mathscr{K} \subset M$ such that $\gamma$ does not cut the good slice $\Sigma:=I^{+}(\mathscr{K}, \tilde{M}) \cap \mathscr{I}^{+}$of $\mathscr{I}^{+}$. Then either $|\gamma| \subset I^{+}(\mathscr{K}, \tilde{M})$ or $|\gamma| \subset \tilde{M}-$ $I^{+}(\mathscr{K}, \tilde{M})$. In fact, since $\gamma$ is endless in $\tilde{M}$ and Lemma 4.2 therefore gives $\mathscr{K} \subset M \subset I^{-}(|\gamma|, \tilde{M})$, the only possibility is $|\gamma| \subset I^{+}(\mathscr{K}, \tilde{M})$. Let $\mu$ be a past endless segment of $\gamma$ to some point $r \in|\gamma|$. Construct the closed past set $\mathscr{P}:=\bigcap_{x \in|\mu|} \bar{I}^{-}(x, \tilde{M})$ of $(\tilde{M}, \tilde{\mathbf{g}})$.

Suppose there exists $q \in \overline{|\mu|} \cap \mathscr{P}$. Since (II) gives $L^{-}(\mu, \tilde{M})=L^{-}(\gamma, \tilde{M})=\varnothing$, the set $|\mu|$ is closed and one has $q \in|\mu| \cap \mathscr{P}$. Let $p \in J^{-}(q,|\mu|)$ be distinct from $q$. Then $q \in \bar{I}^{-}(p, \tilde{M})$. Let $\mathscr{N}_{p}$ be a closed neighbourhood of $p$ not containing $q$. Let $\mathscr{N}_{p}^{\prime} \subset \mathscr{N}_{p}$ be a neighbourhood of $p$. Then $\mathscr{N}_{q}^{\prime}:=I^{+}\left(\mathcal{N}_{p}^{\prime}, \tilde{M}\right)-\mathcal{N}_{p}$ is a neighbourhood of $q$ and there exists $q^{\prime} \in \mathscr{N}_{q}^{\prime} \cap I^{-}(p, \tilde{M})$. It follows that there exists a timelike curve from $\mathscr{N}_{p}^{\prime}$ through $q^{\prime} \in \mathscr{N}_{q}^{\prime} \subset \tilde{M}-\mathscr{N}_{p}$ to $p \in \mathscr{N}_{p}^{\prime}$. This gives a contradiction since strong causality holds at $p \in|\mu| \subset \mathscr{I}_{0}^{+}$. Hence $\overline{|\mu|} \cap \mathscr{P}$ is empty.

The inclusions $|\mu| \subset|\gamma| \subset I^{+}(\mathscr{K}, \tilde{M})$ imply that $\mathscr{P} \cap \mathscr{K}=\bigcap_{x \in|\mu|}\left(\bar{I}^{-}(x, \tilde{M}) \cap \mathscr{K}\right)$ is a monotone intersection of non-empty closed subsets of the compact set $\mathscr{K}$ and is therefore non-empty. However $\mathscr{P}$ does not intersect $\overline{|\mu|}$ so one must have $\dot{\mathscr{P}} \neq \varnothing$. Since a future endpoint of a generator of $\dot{\mathscr{P}}$ would necessarily lie in $\overline{|\dot{\mu}|} \cap \dot{\mathscr{P}} \subset \overline{|\mu|} \cap \mathscr{P}=\varnothing$, every generator of $\dot{\mathscr{P}}$ is future endless in $\tilde{M}$ and is a generating segment of either $\mathscr{I}^{+}$or $\mathscr{I}^{-}$. Hence $\dot{\mathscr{P}} \cap M=\varnothing$. The set $\mathscr{P}$, having non-empty intersection with $\mathscr{K} \subset M$, therefore contains all of $M$ and, being closed in $\tilde{M}$, is coincident with $\tilde{M}$. This contradicts $\overline{|\mu|} \cap \mathscr{P}=\varnothing$.

Corollary. $\mathscr{I}_{0}^{+}$is an invariant open submanifold of the flow of the null geodesic generators of $\mathscr{I}^{+}$.

Proof. Immediate by Proposition 4.13(I).

Note that the relative boundary of $\mathscr{I}_{0}^{+}$in $\mathscr{I}^{+}$may be badly behaved, so $\overline{\mathscr{I}}_{0}^{+}$ need not be a topological submanifold-with-boundary of $\mathscr{I}^{+}$.

Lemma 4.14. Let $\mathscr{K}$ be a compact set of $M$ and let $\Sigma:=\dot{I}^{+}(\mathscr{K}, \tilde{M}) \cap \mathscr{I}^{+}$. Then $\mathscr{I}^{+}-I^{+}(\mathscr{K}, \tilde{M})=J^{-}\left(\Sigma, \mathscr{I}^{+}\right) \subset \mathscr{I}_{0}^{+}$.

Proof. Let $p \in \mathscr{I}^{+}-I^{+}(\mathscr{K}, \tilde{M})$. Then $\mathscr{K} \not I^{-}(p, \tilde{M})$ and hence $M \not I^{-}(p, \tilde{M})$, so strong causality must hold at $p$. Lemma 4.13(III) gives that the generator $\gamma$ of $\mathscr{I}_{0}^{+}$ through $p$ cuts $\Sigma$. If $p \notin \Sigma=\dot{I}^{+}(\mathscr{K}, \tilde{M}) \cap \mathscr{I}^{+}$then $p \notin \bar{I}^{+}(\mathscr{K}, \tilde{M})=\bar{J}^{+}(\mathscr{K}, \tilde{M})=$ $J^{+}(\mathscr{K}, \tilde{M})$, by Lemma 3.3(I) and Lemma 4.5(I), and there can be no causal curve from $\Sigma \subset J^{+}(\mathscr{K}, \tilde{M})$ to $p \notin J^{+}(\mathscr{K}, \tilde{M})$. In general therefore $\gamma$ admits a, possibly degenerate, segment from $p$ to $\Sigma$. There follows $p \in J^{-}\left(\Sigma, \mathscr{I}^{+}\right)$and more generally $\mathscr{I}^{+}-I^{+}(\mathscr{K}, \widetilde{M}) \subset J^{-}\left(\Sigma, \mathscr{I}^{+}\right)$.

Let $q \in J^{-}\left(\Sigma, \mathscr{I}^{+}\right)$. Then there exists $r \in \Sigma \cap J^{+}(q, \tilde{M})$. One cannot have 
$q \in I^{+}(\mathscr{K}, \tilde{M})$ for this would imply $r \in I^{+}(\mathscr{K}, \tilde{M})$ which is incompatible with $r \in \Sigma \subset \dot{I}^{+}(\mathscr{K}, \tilde{M})$. Hence $q \notin I^{+}(\mathscr{K}, \tilde{M})$ and more generally $J^{-}\left(\Sigma, \mathscr{I}^{+}\right) \subset \mathscr{I}^{+}-$ $I^{+}(\mathscr{K}, \tilde{M})$.

Lemma 4.15. If $\Sigma$ is a good slice of $\mathscr{I}^{+}$then $\mathscr{I}^{-} \subset I^{-}(\Sigma, \tilde{M})$.

Proof. Since $I^{-}(\Sigma, \tilde{M})$ intersects $\mathscr{I}^{-}$it will suffice to show that $\dot{I}^{-}(\Sigma, \tilde{M})$ does not. Suppose, to the contrary, that there exists $p \in \dot{I}^{-}(\Sigma, \tilde{M}) \cap \mathscr{I}^{-}$. One cannot have $M \subset I^{+}(p, \tilde{M})$ for this would imply $\Sigma \subset I^{+}(p, \tilde{M})$ and hence $p \in I^{-}(\Sigma, \tilde{M})$. Strong causality must therefore hold at $p$. Let $\mu$ be the future endless generating segment of $\mathscr{I}^{-}$from $p$. Proposition 4.13(I) gives that $\mu$ is a generating segment of $\mathscr{I}_{0}^{-}$.

Consider the closed future set $\mathscr{F}:=\bigcap_{x \in|\mu|} \bar{I}^{+}(x, \tilde{M})$ of $(\tilde{M}, \tilde{\mathbf{g}})$. If there was to exist $r \in\left(J^{-}\left(\Sigma, \mathscr{I}^{+}\right)-\Sigma\right) \cap \mathscr{F}$ then $J^{-}(\Sigma, \tilde{M})$ would be a neighbourhood of $r \in \bar{I}^{+}(p, \tilde{M})$ and so would intersect $I^{+}(p, \tilde{M})$. This would imply $p \in I^{-}(\Sigma, \tilde{M})$, giving a contradiction. Hence $J^{-}\left(\Sigma, \mathscr{I}^{+}\right)-\Sigma$ does not intersect $\mathscr{F}$.

Suppose there exists $s \in \overline{|\mu|} \cap \mathscr{F}$. Since Proposition 4.13(II) gives $L^{+}(\mu, \tilde{M})=\varnothing$, the set $|\mu|$ is closed in $\tilde{M}$ and one has $s \in|\mu| \cap \mathscr{F}$. Let $t \in J^{+}(s,|\mu|)$ be distinct from $s$. Then $s \in \bar{I}^{+}(t, \tilde{M})$. Let $\mathscr{N}_{t}$ be a closed neighbourhood of $t$ not containing $s$. Let $\mathcal{N}_{t}^{\prime} \subset \mathcal{N}_{t}$ be a neighbourhood of $t$. Then $\mathscr{N}_{s}^{\prime}:=I^{-}\left(\mathscr{N}_{t}^{\prime}, \tilde{M}\right)-\mathscr{N}_{t}$ is a neighbourhood of $s$ and there exists $s^{\prime} \in \mathscr{N}_{s}^{\prime} \cap I^{+}(t, \tilde{M})$. Hence there exists a timelike curve from $t \in \mathscr{N}_{t}^{\prime}$ through $s^{\prime} \in \mathcal{N}_{s}^{\prime} \subset \tilde{M}-\mathcal{N}_{t}$ to $\mathcal{N}_{t}^{\prime}$. This gives a contradiction since strong causality holds at $t \in|\mu| \subset \mathscr{I}_{0}^{-}$. Hence $\overline{|\mu|} \cap \mathscr{F}=\varnothing$.

By hypothesis one has $\Sigma=\dot{J}^{+}(\mathscr{K}, \tilde{M}) \cap \mathscr{I}^{+}$for some compact set $\mathscr{K} \subset M$. Let $x \in|\mu| \subset \mathscr{I}_{0}^{-}$and let $y \in I^{+}(x, \tilde{M}) \cap M$. Since every generator of $\mathscr{I}_{0}^{+}$cuts both $\Sigma$ and $\Sigma_{y}:=\dot{J}^{+}(y, \tilde{M}) \cap \mathscr{I}^{+} \subset I^{+}(x, \tilde{M})$, there exists a timelike curve from $x \in \tilde{M}-$ $J^{+}(\mathscr{K}, \tilde{M})$ to $J^{+}(\Sigma, \tilde{M}) \subset \mathrm{J}^{+}(\mathscr{K}, \tilde{M})$ and hence a timelike curve from $x$ to $j^{+}(\mathscr{K}, \tilde{M})$. Thus $\mathscr{F} \cap \dot{J}^{+}(\mathscr{K}, \tilde{M})=\bigcap_{x \in|\mu|}\left(\bar{I}^{+}(x, \tilde{M}) \cap \dot{J}^{+}(\mathscr{K}, \tilde{M})\right)$ is a monotone intersection of non-empty closed subsets of the compact set $j^{+}(\mathscr{K}, \tilde{M})$ and so is non-empty. However $\mathscr{F}$ does not intersect $\overline{|\mu|}$, so $\dot{\mathscr{F}}$ must be non-empty also. Since a past endpoint of a generator of $\dot{\mathscr{F}}$ would necessarily lie in $\overline{|\mu|} \cap \dot{\mathscr{F}} \subset \overline{|\mu|} \cap \mathscr{F}=\varnothing$, every generator of $\dot{\mathscr{F}}$ is past endless in $\tilde{M}$ and is a generating segment of either $\mathscr{I}^{+}$or $\mathscr{I}^{-}$. Hence $\dot{\mathscr{F}} \cap M=\varnothing$. If the closed set $\mathscr{F}$ contained any point of the dense set $M$ of $\tilde{M}$ one would therefore have $\mathscr{F}=\tilde{M}$, which contradicts $\dot{\mathscr{F}} \neq \varnothing$. Thus $\mathscr{F}=\dot{\mathscr{F}} \subset \partial \tilde{M}$. However a generator of $\dot{\mathscr{F}}$ through a point of $\dot{\mathscr{F}} \cap \Sigma=\mathscr{F} \cap \Sigma=$ $\mathscr{F} \cap\left(\dot{J}^{+}(\mathscr{K}, \tilde{M}) \cap \partial \tilde{M}\right)=(\mathscr{F} \cap \partial \tilde{M}) \cap \dot{J}^{+}(\mathscr{K}, \tilde{M})=\mathscr{F} \cap \dot{J}^{+}(\mathscr{K}, \tilde{M}) \neq \varnothing$ would have to cut $J^{-}\left(\Sigma, \mathscr{I}^{+}\right)-\Sigma$. This is impossible since $J^{-}\left(\Sigma, \mathscr{I}^{+}\right)-\Sigma$ does not intersect $\mathscr{F}=\dot{\mathscr{F}}$.

It will be evident that the proofs of many of the previous results could have been simplified if $(\tilde{M}, \tilde{\mathbf{g}})$ was causally simple, that is if $J^{+}(p, \tilde{M})$ and $J^{-}(p, \tilde{M})$ were closed in $\tilde{M}$ for all $p \in \tilde{M}$. However this need not generally be the case. Suppose first that the causality condition holds at a point $p \in \mathscr{I}^{+}$, but that the future endless generating segment $\gamma_{p}^{+}$of $\mathscr{I}^{+}$from $p$ is such that $L^{+}\left(\gamma_{p}^{+}, \tilde{M}\right) \neq \varnothing$. Then strong causality is violated at every point of $L^{+}\left(\gamma_{p}^{+}, \tilde{M}\right) \subset \mathscr{I}^{+}$and, since $J^{+}(p, \tilde{M})=\left|\gamma_{p}^{+}\right|$ is not a closed subset of $\tilde{M},(\tilde{M}, \tilde{\mathbf{g}})$ is not causally simple. Suppose now that strong causality holds at both $\mathscr{I}^{+}$and $\mathscr{I}^{-}$. Let $q \in \mathscr{I}^{-}$. Then every generator of $\dot{J}^{+}(q, \tilde{M})$ 
which cuts $M \cup \mathscr{I}^{-}$must have a past endpoint at $q$. However it is conceivable that a generator of $\dot{J}^{+}(q, \tilde{M})$ could also be a past endless generating segment $\lambda$ of $\mathscr{I}^{+}$. In such a circumstance the set $J^{+}(q, \tilde{M})$ would not intersect $|\lambda| \subset j^{+}(q, \tilde{M})$, except possibly at a future endpoint of $\lambda$, and so would not be closed in $\tilde{M}$. Thus, contrary to a claim in [7], strong causality at $\mathscr{I}^{+}$and $\mathscr{I}^{-}$does not guarantee the causal simplicity of $(\tilde{M}, \tilde{\mathbf{g}})$.

\section{Topological Structure of Simple Space-Times}

There are various assertions in the literature of relevance to the topological structure of simple space-times. However some are based on deficient or incorrect arguments. Consider first Penrose's attempt [1, Appendix] to show that $\mathscr{I}^{+}$has topology $\mathbb{S}^{2} \times \mathbb{R}$ independently of any causality condition on $\mathscr{I}^{+}$. Having chosen an arbitrary point $Q \in M$, he considers the set $\mathscr{C}$ of all points of $\tilde{M}$ lying on null geodesics from $Q$ to $\mathscr{I}^{+}$, and the compact embedded 3-submanifold-with-boundary $\mathscr{B}$ of $\tilde{M}$ defined, in modern notation, as the set $\dot{I}^{+}(Q, \tilde{M})$. With the objective of obtaining a contradiction, Penrose supposes that there is a generator $h$ of $\mathscr{I}^{+}$which cuts $\mathscr{C}$ at some point $R$, but which does not cut $\mathscr{B}$. Using his constructions and notation, one has that the null geodesics $g(S)$ through the variable point $S \in S_{1} R$ all cut $\mathscr{B}$, and that the points at which they do so accumulate at some point $T \in \mathscr{B} \cap \mathscr{I}^{+}$. The generator $h$ of $\mathscr{I}^{+}$through $R$ is a limit curve of the $g(S)$, as is the generator $t$ of $\mathscr{I}^{+}$through $T$. But as $S$ approaches $R$, the segment of $g(S)$ between $\mathscr{B}$ and $S$ may become arbitrarily long, and so no contradiction need arise from the consequence $t \neq h$ of the hypothesis that $h$ does not cut $\mathscr{B}$. This error, which alone invalidates the proof, is compounded by another of an even more fundamental nature. The set of all generators of $\mathscr{I}^{+}$which cut $\mathscr{B} \cap \mathscr{I}^{+}=\partial \mathscr{B}$ foliate an open submanifold of $\mathscr{I}^{+}$homeomorphic to $\partial \mathscr{B} \times \mathbb{R}$. On the grounds that $\partial \mathscr{B}$ is a compact 2-manifold (without boundary), Penrose makes a false inference that this region of $\mathscr{I}^{+}$must be disconnected from the complementary region $\mathscr{I}^{*}$. All that is clear is that $\mathscr{I}^{*}$ is closed, with its topological boundary in $\mathscr{I}^{+}$foliated by a collection of generators of $\mathscr{I}^{+}$which do not cut $\partial \mathscr{B}$. Evidently there is no basis for Penrose's claim that $\mathscr{I}^{*}$ is empty and that $\mathscr{I}^{+}$is consequently homeomorphic to $\partial \mathscr{B} \times \mathbb{R}$.

An article of Geroch [2] gave a correct proof that one could conclude $\mathscr{I}^{+} \approx \mathbb{S}^{2} \times \mathbb{R}$ under an assumption that the topology of $\mathscr{I}^{+}$is of the form $K \times \mathbb{R}$ for some compact 2-manifold $K$. This rather strong assumption was weakened by Hawking \& Ellis [3] to strong causality at $\mathscr{I}^{+}$. Both discussions implicitly assumed orientability of the space-time manifold $M$, and Hawking \& Ellis implicitly assumed the conformal completion of the space-time to be causally simple. Geroch also gave some reason to believe that every Cauchy surface $\mathscr{C}$ of the space-time must have topology $\mathbb{R}^{3}$, and his argument was reproduced by Hawking $\&$ Ellis. Geroch noted that the assumption that $M$ is orientable implies the orientability, and hence parallelizability, of the 3-manifold $\mathscr{C}$, and that this implies the triviality of the bundle $N_{\mathscr{L}}$. No mention was made of the fact, established below, that $N_{+}$must be a non-trivial $\mathbb{R}^{2}$ bundle over $\mathscr{I}^{+} \approx \mathbb{S}^{2} \times \mathbb{R}$. Nonetheless it will be seen later that one does, under Geroch's hypotheses, have $N_{+} \approx T \mathbb{S}^{2} \times \mathbb{R} \approx \mathbb{S}^{2} \times \mathbb{R}^{3}$. The claim that the homeomorphism $N_{\mathscr{C}} \approx N_{+}$implies $\mathscr{C} \times \mathbb{S}^{2} \approx \mathbb{R}^{3} \times \mathbb{S}^{2}$ is therefore correct. 
However he then claimed, without justification, that this implies $\mathscr{C} \approx \mathbb{R}^{3}$. The inference is certainly false if the Poincare conjecture is false (see Sect. 6). Suppose then that the Poincare conjecture is true. The homeomorphism $\mathscr{C} \times \mathbb{S}^{2} \approx \mathbb{R}^{3} \times \mathbb{S}^{2}$ implies $\pi_{r}(\mathscr{C}) \oplus \pi_{r}\left(\mathbb{S}^{2}\right) \cong \pi_{r}\left(\mathbb{S}^{2}\right)$ for all $r \geqq 0$. Since the homotopy groups of $\mathbb{S}^{2}$ are all finitely generated, (Spanier [4, p. 516]), the homotopy groups of $\mathscr{C}$ must therefore be trivial. Hence $\mathscr{C}$ is a contractible open 3 -manifold. It then follows by van Kampen's theorem that $\mathscr{C}$ is irreducible (i.e. every tamely embedded 2-sphere bounds a 3-disc). The homeomorphism $\mathscr{C} \times \mathbb{S}^{2} \approx \mathbb{R}^{3} \times \mathbb{S}^{2}$ also implies that $\mathscr{C}$ is simply connected at infinity (i.e. for every compact set $\mathscr{K}_{1}$ of $\mathscr{C}$, there exists a compact set $\mathscr{K}_{2} \supset \mathscr{K}_{1}$ of $\mathscr{C}$ such that $\pi_{1}\left(\mathscr{C}-\mathscr{K}_{2}\right) \rightarrow \pi_{1}\left(\mathscr{C}-\mathscr{K}_{1}\right)$ is trivial). The properties of irreducibility, contractibility and simple connectivity at infinity are sufficient to guarantee that $\mathscr{C}$ is homeomorphic to $\mathbb{R}^{3}$ (Scott [8]). (Proof. Let $\mathscr{K}_{1}$ and $\mathscr{K}_{2}$ be as above. Perform surgery on $\mathscr{K}_{2}$ outside $\mathscr{K}_{1}$ to produce a compact $\mathscr{K}_{2}^{\prime} \supset \mathscr{K}_{1}$ such that $\pi_{1}\left(\partial \mathscr{K}_{2}^{\prime}\right) \rightarrow$ $\pi_{1}\left(\mathscr{K}_{2}^{\prime}\right)$ and $\pi_{1}\left(\partial \mathscr{K}_{2}^{\prime}\right) \rightarrow \pi_{1}\left(\mathscr{C}-\mathscr{K}_{2}^{\prime}\right)$ are trivial. Homological considerations then give that $\partial \mathscr{K}_{2}^{\prime}$ is a disjoint union of 2-spheres, one of which bounds a 3-disc containing $\mathscr{K}_{1}$. Thus $\mathscr{C}$ is a monotone union of open 3 -cells, and so is itself an open 3-cell.) Geroch's proof that the Cauchy surface $\mathscr{C}$ is homeomorphic to $\mathbb{R}^{3}$ is therefore correct iff the Poincare conjecture is correct.

More recently, Newman \& Clarke [7] gave a different argument to establish the topology of the Cauchy surfaces. Strong causality was again assumed to hold at $\mathscr{I}^{+}$and $\mathscr{I}^{-}$, but the space-time manifold was not required to be orientable. The proof did make use of an erroneous assertion that the conformal completion of the space-time is necessarily causally simple, but this defect may be rectified by means of the results of the previous section of the present paper. Use was also made of the Poincaré conjecture, thought at the time to have been proved by Rêgo and Rourke [9]. Without this, one has that $\mathscr{I}^{+}$and $\mathscr{I}^{-}$have topology $\mathbb{S}^{2} \times \mathbb{R}$, and that every Cauchy surface is homeomorphic to the complement of a point in a homotopy 3-sphere. If the Poincaré conjecture is true after all, it follows that the Cauchy surfaces must have topology $\mathbb{R}^{3}$.

The following theorem supercedes all of the previous work. Strong causality is not required to hold at $\mathscr{I}^{+}$or $\mathscr{I}^{-}$, the space-time manifold is not required to be orientable, and the Poincaré conjecture is not assumed to be true.

Theorem 5.1. Let $(M, \mathbf{g})$ be a simple space-time and $(\tilde{M}, \tilde{\mathbf{g}})$ an asymptotic null completion of $(M, \mathbf{g})$. Let $\mathscr{C}$ be a smoothly embedded Cauchy surface of $(M, \mathbf{g})$. Let $\mathscr{I}_{0}^{+}$be the strongly causal region of $\mathscr{I}^{+}$. Then

(I) $\mathscr{C}$ is diffeomorphic to the complement of a point in a smooth homotopy 3-sphere $\widetilde{S}^{3}$;

(II) $\mathscr{I}^{+}$is diffeomorphic to the complement of a point in a contractible open 3-manifold $C^{3}$ which embeds in $\widetilde{\mathbb{S}}^{3}$;

(III) $\mathscr{I}_{0}^{+}$is diffeomorphic to the complement of a point in $\mathbb{R}^{3}$;

(IV) every slice of $\mathscr{I}^{+}$is homeomorphic to $\mathbb{S}^{2}$;

(V) every slice of $\mathscr{I}^{+}$is a strong deformation retract of both $\mathscr{I}_{0}^{+}$and $\mathscr{I}^{+}$;

(VI) $M$ is homeomorphic to $\mathbb{R}^{4}$.

Proof. The approach will be as follows. Since slices of $\mathscr{I}^{+}$are only 2-dimensional, the claim (IV) is established first. A proof comes from $\mathbb{Z}_{2}$-homological consi- 
derations. The fact that any good slice of $\mathscr{I}^{+}$is cut by all generators of $\mathscr{I}_{0}^{+}$then implies (III). The next step is to argue that, since slices of $\mathscr{I}^{+}$are simply connected and cannot be unwrapped, the manifold $\tilde{M}$ must be simply connected. It follows that $\mathscr{C}$ is simply connected. Choosing an arbitrary point $p \in M$ one then considers the compact 3-submanifold-with-boundary $\Lambda:=\dot{I}^{+}(p, \tilde{M})$ of $\tilde{M}$. Since $\partial \Lambda$ is a slice of $\mathscr{I}^{+}$, and is therefore homeomorphic to $\mathbb{S}^{2}$, the quotient space $\Lambda / \partial \Lambda$ is a compact topological 3-manifold. The simple connectivity of $\mathscr{C} \approx \AA$ now leads to (I). The homeomorphism between the total spaces of $N_{+}$and $N_{\mathscr{C}}$, as in the proof of Proposition 4.9 , is used to identify the $\mathbb{Z}$-homology of $\mathscr{I}^{+}$and to establish its simple connectivity. One then shows that homeomorphism-type is preserved by the removal of the complement of $J^{+}(p, \tilde{M}) \cap \mathscr{I}^{+}$in $\mathscr{I}^{+}$, and that all reduced homology is annihilated the subsequent adjunction of a closed 3-disc. An appeal to Hurewicz then yields (II). The complement of $J^{+}(p, \tilde{M}) \cap \mathscr{I}^{+}$is in fact contained in $\mathscr{I}_{0}^{+}$, and its removal from the pair $\left(\mathscr{I}^{+}, \mathscr{I}_{0}^{+}\right)$is an excision. This leads, by the argument in support of (II), to the triviality of the relative $\mathbb{Z}$-homology of $\left(\mathscr{I}^{+}, \mathscr{I}_{0}^{+}\right)$. The homeomorphism in (III) is used to establish that every good slice of $\mathscr{I}^{+}$, and therefore every slice $\Sigma$, is a strong deformation retract of $\mathscr{I}_{0}^{+}$. The consequent triviality of the relative $\mathbb{Z}$-homology of the pair $\left(\mathscr{I}^{+}, \Sigma\right)$ implies the vanishing of all obstructions to a strong deformation retraction of $\mathscr{I}^{+}$to $\Sigma$, thus completing the proof of (V). Finally, (VI) will follow from (I).

As in the proof of Proposition 4.9, let $N_{\mathscr{C}}$ be the bundle of future-directed null directions over $\mathscr{C}$, and let $N_{+}$be the bundle of all future-directed null directions not tangent to $\mathscr{I}^{+}$over $\mathscr{I}^{+}$. The fibres of $N_{\mathscr{C}}$ are homeomorphic to $\mathbb{S}^{2}$, whilst those of $N_{+}$are homeomorphic to $\mathbb{S}^{2}-\{p t.\} \approx \mathbb{R}^{2}$. The future-directed null geodesics of $(\tilde{M}, \tilde{\mathbf{g}})$ from $\mathscr{C}$ to $\mathscr{I}^{+}$define a homeomorphism of the total space of $N_{\mathscr{C}}$ onto the total space of $N_{+}$. In fact, all that will be required is the existence of a homotopy equivalence $N_{\mathscr{C}} \rightarrow N_{+}$. Note that the projection $N_{+} \rightarrow \mathscr{I}^{+}$of $N_{+}$onto its base space is another homotopy equivalence.

Remark 5.2. The homotopy sequence for $N_{\mathscr{C}}$ assumes the form

$$
\begin{aligned}
\rightarrow \pi_{r+1}(\mathscr{C}) \rightarrow \pi_{r}\left(\mathbb{S}^{2}\right) \rightarrow & \pi_{r}\left(N_{\mathscr{C}}\right) \rightarrow \pi_{r}(\mathscr{C}) \rightarrow \\
\downarrow & \cong \\
& \pi_{r}\left(N_{+}\right) \\
& \downarrow \cong \\
& \pi_{r}\left(\mathscr{I}^{+}\right)
\end{aligned}
$$

Lemma 5.3. $\mathscr{C}$ has the $\mathbb{Z}_{2}$-homology and $\mathbb{Z}$-homology of a point, and $\mathscr{I}^{+}$has the $\mathbb{Z}_{2}$-homology of $\mathbb{S}^{2}$.

Proof. Since $N_{\mathscr{C}}$ is a 2-sphere bundle over $\mathscr{C}$ one has a Gysin homology sequence

$$
\begin{gathered}
\rightarrow H_{r+1}\left(\mathscr{C} ; \mathbb{Z}_{2}\right) \rightarrow H_{r-2}\left(\mathscr{C} ; \mathbb{Z}_{2}\right) \rightarrow H_{r}\left(N_{\mathscr{C}} ; \mathbb{Z}_{2}\right) \rightarrow H_{r}\left(\mathscr{C} ; \mathbb{Z}_{2}\right) \rightarrow \\
\downarrow \cong \\
H_{r}\left(N_{+} ; \mathbb{Z}_{2}\right) \\
\downarrow \cong \\
H_{r}\left(\mathscr{I}^{+} ; \mathbb{Z}_{2}\right) .
\end{gathered}
$$


Since Proposition 4.9 gives that the 3-manifolds $\mathscr{I}^{+}$and $\mathscr{C}$ are connected and non-compact, working from left to right one finds $H_{r}\left(\mathscr{C} ; \mathbb{Z}_{2}\right) \cong 0$ for all $r \geqq 1, H_{r}\left(\mathscr{I}^{+} ; \mathbb{Z}_{2}\right) \cong 0$ for all $r \geqq 3$ and for $r=1$, with $H_{2}\left(\mathscr{I}^{+} ; \mathbb{Z}_{2}\right) \cong H_{0}\left(\mathscr{I}^{+} ; \mathbb{Z}_{2}\right) \cong$ $H_{0}\left(\mathscr{C} ; \mathbb{Z}_{2}\right) \cong \mathbb{Z}_{2}$. Thus $\mathscr{I}^{+}$has the $\mathbb{Z}_{2}$-homology of $\mathbb{S}^{2}$ and $\mathscr{C}$ has the $\mathbb{Z}_{2}$-homology of a point.

The universal coefficient theorem for homology gives a short exact sequence

$$
0 \rightarrow H_{r}(\mathscr{C}) \otimes \mathbb{Z}_{2} \rightarrow H_{r}\left(\mathscr{C} ; \mathbb{Z}_{2}\right) \rightarrow \operatorname{Tor}\left(H_{r-1}(\mathscr{C}), \mathbb{Z}_{2}\right) \rightarrow 0 .
$$

One thus has $H_{r}(\mathscr{C}) \otimes \mathbb{Z}_{2} \cong \operatorname{Tor}\left(H_{r}(\mathscr{C}), \mathbb{Z}_{2}\right) \cong 0$ for all $r \geqq 1$, and it follows that $\mathscr{C}$ has the $\mathbb{Z}$-homology of a point.

Let $p \in M$. Then Lemmas 3.4 and 4.5 give that $\Lambda:=\dot{I}^{+}(p, \tilde{M})$ is a compact achronal embedded topological 3-submanifold-with-boundary of $\tilde{M}$, with $\partial \Lambda=$ $\dot{I}^{+}(p, \tilde{M}) \cap \mathscr{I}^{+}$.

Lemma 5.4. $\mathscr{C} \approx \AA$.

Proof. Let $\tilde{\mathbf{V}}$ be a continuous, nowhere-zero, non-spacelike vector field on $\tilde{M}$ which is timelike on $M$, and on $\partial \tilde{M}=\mathscr{I}^{+} \cup \mathscr{I}^{+}$is null and tangent thereto. The maximal integral curves of $\tilde{\mathbf{V}} \mid M$ are endless in $\tilde{M}$ and cut $\mathscr{C}$. Moreover, by Lemma 4.2 , they cut $I^{+}(p, \tilde{M}) \cap M$ and $I^{-}(p, \tilde{M}) \cap M \subset M-I^{+}(p, \tilde{M})$, and so cut $\dot{I}^{+}(p, \tilde{M}) \cap M=\grave{\Lambda}$. The maximal integral curves of $\tilde{\mathbf{V}} \mid M$ thus define a homeomorphism of $\Lambda$ onto $\mathscr{C}$.

Remark 5.5. The generators of $\mathscr{I}_{0}^{+}$, when parametrized with respect to arc-length from the good slice $\partial \Lambda$ with respect to a complete positive definite metric on $\mathscr{I}_{0}^{+}$, equip $\mathscr{I}_{0}^{+}$with the structure of a 1 -dimensional Euclidean vector bundle over $\partial \Lambda$ with group $O(1) \cong \mathbb{Z}_{2}$.

Lemma 5.6. $\partial \Lambda$ is homeomorphic to $\mathbb{S}^{2}$ and is a strong deformation retract of $\mathscr{I}_{0}^{+}$.

Proof. The inclusion $\Lambda \rightarrow \Lambda$ is a homotopy equivalence as a consequence of the topological collaring theorem. Since $\mathscr{C} \approx \Lambda$ has the $\mathbb{Z}$-homology of a point, it therefore follows that $\Lambda$ has the $\mathbb{Z}$-homology of a point. The universal coefficient theorem for cohomology then gives that $\Lambda$ has the $\mathbb{Z}_{2}$-cohomology of a point. Since $\Lambda$ is $\mathbb{Z}_{2}$-orientable, Lefschetz duality gives the commutative diagram

$$
\begin{aligned}
& \rightarrow H^{1}\left(\Lambda ; \mathbb{Z}_{2}\right) \quad \rightarrow H^{1}\left(\partial \Lambda ; \mathbb{Z}_{2}\right) \rightarrow H^{1}\left(\Lambda, \partial \Lambda ; \mathbb{Z}_{2}\right) \rightarrow \\
& \downarrow \cong \quad \downarrow \cong \quad \downarrow \cong \\
& \rightarrow H_{2}\left(\Lambda, \partial \Lambda ; \mathbb{Z}_{2}\right) \rightarrow H_{1}\left(\partial \Lambda ; \mathbb{Z}_{2}\right) \rightarrow H_{1}\left(\Lambda ; \mathbb{Z}_{2}\right) \rightarrow \text {. }
\end{aligned}
$$

There follows $H_{1}\left(\partial \Lambda ; \mathbb{Z}_{2}\right) \cong 0$. Each component of $\partial \Lambda$, being compact and $\mathbb{Z}_{2}$ orientable therefore has the $\mathbb{Z}_{2}$-homology of a 2 -sphere. Each such component therefore has Euler characteristic +2 and so is homeomorphic to a 2 -sphere. Hence $\partial \Lambda$ is the disjoint union of $n 2$-spheres, for some integer $n \geqq 1$. This implies that one may adjoin $n$ closed 3-discs to $\Lambda$ to obtain a compact 3-manifold $X$. The Euler characteristics of $\Lambda$ and $X$ are related by $0=\chi(X)=\chi(\Lambda)+n(-1)^{3}=\chi(\Lambda)-n$. Since 
$\Lambda$ has the $\mathbb{Z}$-homology of a point one obtains $\chi(\Lambda)=1$. There follows $n=1$ and hence $\partial \Lambda \approx \mathbb{S}^{2}$. Remark 5.5 implies that $\partial \Lambda$ is a strong deformation retract of $\mathscr{I}^{+}{ }_{0}$.

Corollary 1. Every slice of $\mathscr{I}^{+}$is homeomorphic to $\mathbb{S}^{2}$ and is a strong deformation retract of $\mathscr{I}_{0}^{+}$.

Proof. Let $\Sigma$ be a slice of $\mathscr{I}^{+}$. Every generator of $\mathscr{I}_{0}^{+}$which cuts $\Sigma \subset \mathscr{I}_{0}^{+}$must cut the good slice $\partial \Lambda \subset \mathscr{I}_{0}^{+}$of $\mathscr{I}^{+}$. Since $\Sigma$ is acausal and compact, and $\partial \Lambda$ is acausal and connected, the generators of $\mathscr{I}_{0}^{+}$therefore define a TOP isotopy $G_{\Sigma}: \Sigma \times[0,1] \rightarrow \mathscr{I}_{0}^{+} \times[0,1]$ which throws $\Sigma$ onto $\partial \Lambda$. Hence $\Sigma \approx \partial \Lambda \approx \mathbb{S}^{2}$. Moreover the generators of $\mathscr{I}_{0}^{+}$define a TOP isotopy $G_{\mathscr{U}}: \mathscr{U} \times[0,1] \rightarrow \mathscr{I}_{0}^{+} \times[0,1]$ which throws an open neighbourhood $\mathscr{U}$ of $\Sigma$ in $\mathscr{I}_{0}^{+}$onto an open neighbourhood of $\partial \Lambda$ in $\mathscr{I}_{0}^{+}$. The homotopy $H_{\Sigma}: \Sigma \times[0,1] \rightarrow \mathscr{I}_{0}^{+}$of the inclusion $\Sigma \rightarrow \mathscr{I}_{0}^{+}$, defined by $(x, v) \mapsto y$ such that $G_{\Sigma}(x, v)=(y, v)$, therefore extends to a homotopy $H: \mathscr{I}_{0}^{+} \times[0,1] \rightarrow \mathscr{I}_{0}^{+}$of the identity on $\mathscr{I}_{0}^{+}$. The mapping $H_{1}: \mathscr{I}_{0}^{+} \rightarrow \mathscr{I}_{0}^{+}$is a homotopy equivalence, as is the homeomorphism $H_{1} \mid \Sigma: \Sigma \rightarrow \partial \Lambda$. Both therefore induce isomorphisms of homotopy groups (Spanier [4, p. 386]). One thus has a commutative diagram

$$
\begin{aligned}
& \rightarrow \pi_{r}(\Sigma) \rightarrow \pi_{r}\left(\mathscr{I}_{0}^{+}\right) \rightarrow \pi_{r}\left(\mathscr{I}_{0}^{+}, \Sigma\right) \rightarrow \pi_{r-1}(\Sigma) \rightarrow \pi_{r-1}\left(\mathscr{I}_{0}^{+}\right) \rightarrow \\
& \downarrow \cong \downarrow \subseteq \downarrow \downarrow \downarrow \cong \downarrow \\
& \rightarrow \pi_{r}(\partial \Lambda) \rightarrow \pi_{r}\left(\mathscr{I}_{0}^{+}\right) \rightarrow \pi_{r}\left(\mathscr{I}_{0}^{+}, \partial \Lambda\right) \rightarrow \pi_{r-1}(\partial \Lambda) \rightarrow \pi_{r-1}\left(\mathscr{I}_{0}^{+}\right) \rightarrow
\end{aligned}
$$

in which both rows are exact and the vertical homomorphisms are induced by $H_{1}$. The five lemma now gives an isomorphism $\pi_{r}\left(\mathscr{I}_{0}^{+}, \Sigma\right) \cong \pi_{r}\left(\mathscr{I}_{0}^{+}, \partial \Lambda\right)$ for every $r$. But any strong deformation retraction of $\mathscr{I}_{0}^{+}$to $\partial \Lambda$ induces isomorphisms $\pi_{r}\left(\mathscr{I}_{0}^{+}, \partial \Lambda\right) \cong 0$ for all $r$. One must therefore have $\pi_{r}\left(\mathscr{I}_{0}^{+}, \Sigma\right) \cong 0$ for all $r$. Since $\mathscr{I}_{0}^{+}$ is a 3-manifold, and $\Sigma$ is a topological 2-submanifold-with-boundary of $\mathscr{I}^{+}$, the pair $\left(\mathscr{I}^{+}, \Sigma\right)$ may be equipped with the structure of a relative $\mathrm{CW}$-complex. It therefore follows [4, p. 402] that $\Sigma$ is a strong deformation retract of $\mathscr{I}_{0}^{+}$.

Corollary 2. $\mathscr{I}_{0}^{+} \approx \mathbb{S}^{2} \times \mathbb{R}$.

Proof. Since 1-dimensional Euclidean vector bundles over $\mathbb{S}^{2}$ are classified by $\pi_{1}(O(1)) / \pi_{0}(O(1)) \cong \pi_{1}\left(\mathbb{Z}_{2}\right) / \pi_{0}\left(\mathbb{Z}_{2}\right) \cong 0($ Steenrod [10, p. 99]) all such bundles are equivalent and therefore trivial. Thus the assertion follows directly from the lemma and Remark 5.5.

Lemma 5.7. $\pi_{1}(\mathscr{C}) \cong 0$.

Proof. Let $p: \tilde{M}_{c} \rightarrow \tilde{M}$ be the universal cover of $\tilde{M}$. Then $\tilde{M}_{c}$ is a 4-manifold-withboundary such that $\partial \tilde{M}_{c}=p^{-1}(\partial \tilde{M})$. One forms a space-time-with-boundary $\left(\tilde{M}_{c}, \tilde{\mathbf{g}}_{c}\right)$, with $\tilde{\mathbf{g}}_{c}:=p^{*} \tilde{\mathbf{g}}$. The time orientation on $(\tilde{M}, \tilde{\mathbf{g}})$ induces a time orientation on $\left(\tilde{M}_{c}, \tilde{\mathbf{g}}_{c}\right)$. Since $\partial \tilde{M}$ is null with respect to $\tilde{\mathbf{g}}$, and $p$ is a local isometry, $\partial \tilde{M}_{c}$ is null with respect to $\tilde{\mathbf{g}}_{c}$. Let $M_{c}:=\tilde{M}_{c}-\partial \tilde{M}_{c}, \mathbf{g}_{c}:=p^{*} \mathbf{g}$. It is easily checked that $\left(\tilde{M}_{c}, \tilde{\mathbf{g}}_{c}\right)$ is a $C^{r}$ null asymptote of the space-time $\left(M_{c}, \mathbf{g}_{c}\right)$.

Since any closed timelike curve of $\left(M_{c}, \mathbf{g}_{c}\right)$ would project to a closed timelike 
curve of $(M, \mathbf{g})$, one has that $\left(M_{c}, \mathbf{g}_{c}\right)$ satisfies the chronology condition. Moreover, any null geodesic of $\left(M_{c}, \mathbf{g}_{c}\right)$ without a future (respectively past) endpoint in $\tilde{M}_{c}$ would project to a null geodesic of $(M, \mathbf{g})$ without a future (past) endpoint in $\tilde{M}$. Thus every null geodesic of $\left(M_{c}, \mathbf{g}_{\mathrm{c}}\right)$ has both future and past endpoints in $\tilde{M}_{c}$. It follows that $\left(M_{c}, \mathbf{g}_{c}\right)$ is a simple space-time and that $\left(\tilde{M}_{c}, \tilde{\mathbf{g}}_{c}\right)$ is an asymptotic null completion of $\left(M_{c}, \mathbf{g}_{c}\right)$. One also has $\partial \tilde{M}_{c}=\mathscr{I}_{c}^{+} \cup \mathscr{I}_{c}^{-}$where $\mathscr{I}_{c}^{+}=p^{-1}\left(\mathscr{I}^{+}\right)$and $\mathscr{I}_{c}^{-}=p^{-1}\left(\mathscr{I}^{-}\right)$are the future and past null infinities of $\left(\tilde{M}_{c}, \tilde{\mathbf{g}}_{c}\right)$.

Let $r \in M$. Then $\Sigma:=\dot{I}^{+}(r, \tilde{M}) \cap \mathscr{I}^{+}$is a good slice of $\mathscr{I}^{+}$. Since $\Sigma$ is homeomorphic to $\mathbb{S}^{2}$, which is simply connected, each component of $p^{-1}(\Sigma)$ is homeomorphic to $\mathbb{S}^{2}$ and the multiplicity of $p$ is equal to the cardinal number of the set of components of $p^{-1}(\Sigma)$.

Let $r_{c} \in p^{-1}(r)$. If there was to exist a point $s_{c} \in p^{-1}(\Sigma) \cap I^{+}\left(r_{c}, \tilde{M}_{\mathrm{c}}\right)$ then there would exist a timelike curve $\mu_{\mathrm{c}}$ of $\left(\tilde{M}_{c}, \tilde{\mathbf{g}}_{c}\right)$ from $r_{c}$ to $s_{c}$, and $p \circ \mu_{c}$ would be a timelike curve of $(\tilde{M}, \tilde{\mathbf{g}})$ from $r$ to $p\left(s_{c}\right) \in \Sigma \subset \dot{I}^{+}(r, \tilde{M}) \cap \mathscr{I}^{+} \subset \tilde{M}-I^{+}(r, \tilde{M})$. Since this is impossible one must have $p^{-1}(\Sigma) \subset \mathscr{I}_{c}^{+}-I^{+}\left(r_{c}, \tilde{M}_{c}\right) \subset \mathscr{I}_{c 0}^{+}$, by Lemma 4.14. If there was to exist a non-degenerate causal curve $v_{c}$ of $\left(\tilde{M}_{c}, \tilde{\mathbf{g}}_{c}\right)$ from $p^{-1}(\Sigma)$ to $p^{-1}(\Sigma)$, then $p^{\circ} v_{c}$ would be a non-degenerate causal curve of $(\tilde{M}, \tilde{\mathbf{g}})$ from $\Sigma$ to $\Sigma$. This is impossible since $\Sigma$ is acausal. It follows that $p^{-1}(\Sigma)$ is acausal and that each component of $p^{-1}(\Sigma)$ is a slice of $\mathscr{I}_{c}^{+}$.

Consider the good slice $\Sigma_{c}:=\dot{I}^{+}\left(r_{c}, \tilde{M}\right) \cap \mathscr{I}_{c}^{+}$of $\left(\tilde{M}_{c}, \tilde{\mathbf{g}}_{c}\right)$. For each $t_{c} \in p^{-1}(\Sigma)$, the generator of $\mathscr{I}_{c 0}^{+}$through $t_{c}$ must cut $\Sigma_{c}$. Since $p^{-1}(\Sigma)$ is acausal, the generators of $\mathscr{I}_{c 0}^{+}$thus define a homeomorphism of $p^{-1}(\Sigma)$ onto an open submanifold of $\Sigma_{c}$. Since $\Sigma_{c}$ is connected it follows that each component of $p^{-1}(\Sigma)$, being compact, is mapped onto $\Sigma_{c}$. Hence, for any $u_{c} \in \Sigma_{c}$, the generator of $\mathscr{I}_{c 0}^{+}$through $u_{c}$ must cut every component of $p^{-1}(\Sigma)$. It follows that $p^{-1}(\Sigma)$, being acausal, is connected. Thus $p: \tilde{M}_{c} \rightarrow \tilde{M}$ has unit multiplicity and so is a homeomorphism.

One now has $\pi_{1}(\tilde{M}) \cong \pi_{1}\left(\tilde{M}_{c}\right) \cong 0$. Since the inclusion $M=\tilde{M}-\partial \tilde{M} \rightarrow \tilde{M}$ is a homotopy equivalence, there follows $\pi_{1}(M) \cong \pi_{1}(\tilde{M}) \cong 0$. Moreover, since $\mathscr{C}$ is a Cauchy surface for $(M, \mathbf{g})$ one has $M \approx \mathscr{C} \times \mathbb{R}$ and consequently $\pi_{1}(\mathscr{C}) \cong$ $\pi_{1}(M) \cong 0$.

Corollary 1. $\mathscr{C}$ is a contractible open 3-manifold.

Proof. By Lemma 5.3 one has $H_{r}(\mathscr{C}) \cong 0$ for all $r \geqq 1$. Since one also has $\pi_{1}(\mathscr{C}) \cong \pi_{0}(\mathscr{C}) \cong 0$, the Hurewicz isomorphism theorem gives $\pi_{r}(\mathscr{C}) \cong 0$ for all $r \geqq 0$. The assertion now follows by a standard result [4, p. 402].

Corollary 2. $\pi_{r}\left(\mathscr{I}^{+}\right) \cong \pi_{r}\left(\mathbb{S}^{2}\right)$ for all $r \geqq 0$.

Proof. Immediate by Corollary 1 and Remark 5.2.

Corollary 3. $\mathscr{I}^{+}$has the $\mathbb{Z}$-homology of $\mathbb{S}^{2}$.

Proof. Since $\mathscr{I}^{+}$is simply connected one has $H_{0}\left(\mathscr{I}^{+}\right) \cong H_{1}\left(\mathscr{I}^{+}\right) \cong 0$, whereby Hurewicz gives $H_{2}\left(\mathscr{I}^{+}\right) \cong \pi_{2}\left(\mathscr{I}^{+}\right) \cong \mathbb{Z}$. Since $\mathscr{I}^{+}$is a non-compact 3-manifold one has $H_{r}\left(\mathscr{I}^{+}\right) \cong 0$ for all $r \geqq 3$.

Corollary 1 to Lemma 5.7 is strengthened by the corollary to the following result.

Lemma 5.8. The adjunction space $\Lambda \bigcup_{\partial} \mathbb{D}^{3}$ is homeomorphic to a homotopy 3-sphere $\widetilde{\mathfrak{S}}^{3}$. 
Proof. By Lemmas 5.4 and 5.7 one has that $\Lambda \approx \mathscr{C}$ is simply connected. Since $\Lambda$ is compact, the adjunction space $\Lambda \bigcup_{\partial} \mathbb{D}^{3}$ is therefore a compact simply connected topological 3-manifold and (Hempel [11, p. 26]) must be homotopy equivalent to $\mathbb{S}^{3}$.

Corollary. $\mathscr{C} \approx \widetilde{S}^{3}-\{p t$.$\} .$

Proof. By means of Lemma 5.4 one has $\mathscr{C} \approx \hat{\Lambda} \approx \tilde{S}^{3}-\{p t$. $\}$.

Since $J^{+}(p, \tilde{M})$ is a closed future set of $(\tilde{M}, \tilde{\mathbf{g}})$, Lemma 3.4 gives that $\Gamma:=J^{+}(p, \tilde{M}) \cap \mathscr{I}^{+}$is a topological 3-submanifold-with-boundary of $\mathscr{I}^{+}$such that $\partial \Gamma=\dot{J}^{+}(p, \tilde{M}) \cap \mathscr{I}^{+}=\dot{I}^{+}(p, \tilde{M}) \cap \mathscr{I}^{+}$. Moreover one has $\stackrel{\circ}{\Gamma}=I^{+}(p, \tilde{M}) \cap \mathscr{I}^{+}$.

Lemma 5.9. $\Gamma$ is a strong deformation retract of $\mathscr{I}^{+}$such that $\stackrel{\Gamma}{\Gamma} \approx \mathscr{I}^{+}$.

Proof. For any $q \in \mathscr{I}^{+}-\mathscr{I}_{0}^{+}$one has $p \in M \subset I^{-}(q, \tilde{M})$ and hence $q \in I^{+}(p, \tilde{M}) \cap \mathscr{I}^{+}=$

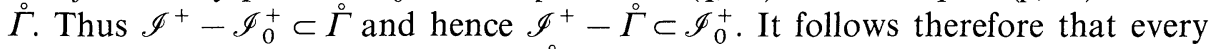
generator of $\mathscr{I}^{+}$which cuts $\mathscr{I}^{+}-\stackrel{\circ}{\Gamma}$ is a generator of $\mathscr{I}_{0}^{+}$and so must cut the good slice $\partial \Gamma$ of $\mathscr{I}^{+}$. Thus the past endless past-directed generating segments of $\mathscr{I}_{0}^{+}$from $\partial \Gamma$, parameterized by arc-length from $\partial \Gamma$ with respect to a complete positive definite metric on $\mathscr{I}^{+}$, define a homeomorphism of $\mathscr{I}^{+}-\stackrel{\circ}{\Gamma}$ onto $\partial \Gamma \times[0, \infty)$. The result follows.

Lemma 5.10. The adjunction space $\Gamma \bigcup_{\partial} \mathbb{D}^{3}$ is homeomorphic to a contractible open 3-manifold $C^{3}$.

Proof. Since $\stackrel{\circ}{\Gamma} \approx \mathscr{I}^{+}$is simply connected, the adjunction space $\Gamma \bigcup_{\partial} \mathbb{D}^{3}$ is a simply connected 3-manifold. If $\Gamma \bigcup_{\partial} \mathbb{D}^{3}$ was compact it would be a homotopy 3-sphere. Mayer-Vietoris would then give $\widetilde{H}_{*}(\Gamma) \cong 0$ which, by the simple connectivity of $\Gamma$ and the Hurewicz isomorphism theorem, would imply $\pi_{*}(\Gamma) \cong 0$. One would then have $\pi_{*}\left(\mathscr{I}^{+}\right) \cong \pi_{*}(\stackrel{\circ}{\Gamma}) \cong \pi_{*}(\Gamma) \cong 0$ which would be incompatible with Corollary 2 to Lemma 5.7. Thus $\Gamma \bigcup_{\hat{0}} \mathbb{D}^{3}$ is non-compact.

The simple connectivity and non-compactness of $\Gamma \bigcup_{\partial} \mathbb{D}^{3}$ imply $H_{1}\left(\Gamma \bigcup_{\partial} \mathbb{D}^{3}\right) \cong$ $H_{3}\left(\Gamma \bigcup_{\hat{c}} \mathbb{D}^{3}\right) \cong H^{3}\left(\Gamma \bigcup_{\hat{c}} \mathbb{D}^{3}\right) \cong 0$. The universal coefficient theorem for cohomology gives $H^{r+1}\left(\Gamma \bigcup^{3} \mathbb{D}^{3}\right) \cong \operatorname{Hom}\left(H_{r+1}\left(\Gamma \bigcup \mathbb{D}^{3}\right), \mathbb{Z}\right) \oplus \operatorname{Ext}\left(H_{r}\left(\Gamma \bigcup \mathbb{D}^{3}\right)^{3}, \mathbb{Z}\right)$ for all $r$, so one has $\operatorname{Ext}\left(H_{2}\left(\Gamma \bigcup_{\partial} \mathbb{D}^{3}\right), \mathbb{Z}\right) \cong 0$. The Mayer-Vietoris sequence for the triad $\left(\Gamma \bigcup_{\partial} \mathbb{D}^{3}, \Gamma, \mathbb{D}^{3}\right)$ now gives a short exact sequence

$$
\begin{array}{cc}
0 \rightarrow H_{2}(\partial \Gamma) \rightarrow & H_{2}(\Gamma) \rightarrow H_{2}\left(\Gamma \bigcup_{o} \mathbb{D}^{3}\right) \rightarrow 0 \\
\downarrow & \cong \quad \downarrow \cong \\
\mathbb{Z} & \mathbb{Z}
\end{array}
$$

which implies that $H_{2}\left(\Gamma \bigcup_{\partial} \mathbb{D}^{3}\right)$ is a finitely generated torsion module. Hence 
$H_{2}\left(\Gamma \bigcup \mathbb{D}^{3}\right) \cong \operatorname{Hom}\left(H_{2}\left(\Gamma \bigcup \mathbb{D}^{3}\right), \mathbb{Z}\right) \oplus \operatorname{Ext}\left(H_{2}\left(\Gamma \bigcup_{\hat{\partial}} \mathbb{D}^{3}\right), \mathbb{Z}\right) \cong 0$. One now has $\tilde{H}_{r}\left(\Gamma \bigcup \mathbb{D}^{3}\right) \cong 0$ for all $r \geqq 0$ whereby the Hurewicz isomorphism theorem gives $\pi_{r}\left(\Gamma \bigcup^{\hat{o}} \mathbb{D}^{3}\right) \cong 0$ for all $r \geqq 0$. There is consequently no obstruction to the contraction of $\Gamma \bigcup_{i}^{i} \mathbb{D}^{3}$.

Corollary. $\mathscr{I}^{+} \approx C^{3}-\{p t$.$\} .$

Lemma 5.11. $C^{3}$ admits a topological embedding into $\widetilde{\mathbb{S}}^{3}$.

Proof. Let $\tilde{\mathbf{W}}$ be a timelike vector field on $(\tilde{M}, \tilde{\mathbf{g}})$. Any maximal integral curve of $\tilde{\mathbf{W}}$ to $\Gamma \subset J^{+}(p, \tilde{M})$ either has a past endpoint at $\mathscr{I}^{-} \subset \tilde{M}-J^{+}(p, \tilde{M})$, or is past endless in $\tilde{M}$ and therefore cuts $I^{-}(p, \tilde{M}) \subset \tilde{M}-J^{+}(p, \tilde{M})$. In both cases it cuts $j^{+}(p, \tilde{M})=\Lambda$. The maximal integral curves of $\tilde{\mathbf{W}}$ to $\Gamma$ therefore define a topological embedding of $\Gamma$ into $\Lambda$. Clearly $\partial \Gamma=\partial \Lambda$ is mapped onto itself. The adjunction space $\Gamma \bigcup_{\partial} \mathbb{D}^{3}$ therefore admits a topological embedding into $\Lambda \bigcup_{\partial} \mathbb{D}^{3}$. The result now follows by Lemmas 5.8 and 5.10 .

Lemma 5.12. Every slice of $\mathscr{I}^{+}$is a strong deformation retract of $\mathscr{I}^{+}$.

Proof. Let $\Sigma$ be a slice of $\mathscr{I}^{+}$. Since Corollary 1 to Lemma 5.6 gives that $\Sigma$ and $\partial \Gamma$ are strong deformation retracts of $\mathscr{I}_{0}^{+}$, and Lemma 5.9 gives that $\partial \Gamma$ is a strong deformation retract of $\mathscr{I}^{+}-\stackrel{\circ}{\Gamma}$, the homology sequences for the triples $\left(\mathscr{I}^{+}, \mathscr{I}_{0}^{+}, \Sigma\right),\left(\mathscr{I}^{+}, \mathscr{I}_{0}^{+}, \partial \Gamma\right)$ and $\left(\mathscr{I}^{+}, \mathscr{I}^{+}-\stackrel{\circ}{\Gamma}, \partial \Gamma\right)$ give $H_{*}\left(\mathscr{I}^{+}, \Sigma\right) \cong H_{*}\left(\mathscr{I}^{+}, \mathscr{I}_{0}^{+}\right) \cong$ $H_{*}\left(\mathscr{I}^{+}, \partial \Gamma\right) \cong H_{*}\left(\mathscr{I}^{+}, \mathscr{I}^{+}-\stackrel{\circ}{\Gamma}\right)$. One thus has $H_{*}\left(\mathscr{I}^{+}, \Sigma\right) \cong H_{*}(\Gamma, \partial \Gamma)$ by excision of $\mathscr{I}^{+}-\Gamma$. But Lemma 5.10 implies $H_{*}(\Gamma, \partial \Gamma) \cong H_{*}\left(C^{3},\{p t\}.\right) \cong \tilde{H}_{*}\left(C^{3}\right) \cong 0$ so one obtains $H_{*}\left(\mathscr{I}^{+}, \Sigma\right) \cong 0$. The simple connectivity of $\mathscr{I}^{+}$and $\Sigma$ permits the application of the relative Hurewicz isomorphism theorem (Spanier [4, p. 397]) to yield $\pi_{*}\left(\mathscr{I}^{+}, \Sigma\right) \cong 0$. It therefore follows [4, p. 402] that $\Sigma$ is a strong deformation retract of $\mathscr{I}^{+}$.

The preceding lemmas may now be brought together to establish the main result. Since all DIFF structures on any topological 3-manifold are isotopic, the Corollary to Lemma 5.8 gives (I), the Corollary to Lemma 5.10 with Lemma 5.11 give (II), and Corollary 2 to Lemma 5.6 gives (III). The claims (IV) and (V) are established by Corollary 1 to Lemma 5.6 and Lemma 5.12. By (I) one has $M \approx_{\mathrm{diff}}\left(\widetilde{\mathbb{S}}^{3}-\{p t\}.\right) \times \mathbb{R}$. Since $\widetilde{\mathbb{S}}^{3}-\{p t$.$\} is proper homotopy equivalent to$ $\mathbb{S}^{3}-\{p t.\} \approx \mathbb{R}^{3}, M$ is proper homotopy equivalent to $\mathbb{R}^{4}$, and a theorem of Freedman [12] gives $M \approx \mathbb{R}^{4}$. This establishes (VI).

Corollary 1. $\mathscr{I}^{+}-\mathscr{I}_{0}^{+}$has no compact component.

Proof. Let $\Sigma$ be a slice of $\mathscr{I}^{+}$. Then $\Sigma$ is a strong deformation retract of both $\mathscr{I}^{+}$and $\mathscr{I}_{0}^{+}$and one has $H_{*}\left(\mathscr{I}^{+}, \Sigma\right) \cong H_{*}\left(\mathscr{I}_{0}^{+}, \Sigma\right) \cong 0$. The homology sequence for the triple $\left(\mathscr{I}^{+}, \mathscr{I}_{0}^{+}, \Sigma\right)$ thus yields $H_{*}\left(\mathscr{I}^{+}, \mathscr{I}_{0}^{+}\right) \cong 0$. In particular one has $H_{3}\left(\mathscr{I}^{+}, \mathscr{I}_{0}^{+}\right) \cong 0$ which, since $\mathscr{I}_{0}^{+}$is relatively open in $\mathscr{I}^{+}$, gives that there are no non-zero compactly supported, continuous $\mathbb{Z}$-valued functions on $\mathscr{I}^{+}-\mathscr{I}_{0}^{+}$.

Suppose $\mathscr{I}^{+}-\mathscr{I}_{0}^{+}$has a compact component $\mathscr{K}$. The characteristic function 
$\chi_{\mathscr{K}}$ of $\mathscr{K}$ in $\mathscr{I}^{+}-\mathscr{I}_{0}^{+}$, regarded as a $\mathbb{Z}$-valued function on $\mathscr{I}^{+}-\mathscr{I}_{0}^{+}$, is clearly compactly supported. Moreover $\chi_{\mathscr{K}}$ is continuous since both $\chi_{\mathscr{K}}^{-1}(1)=\mathscr{K}$ and $\chi_{\mathscr{K}}^{-1}(0)=\left(\mathscr{I}^{+}-\mathscr{I}_{0}^{+}\right)-\mathscr{K}$ are relatively open in $\mathscr{I}^{+}-\mathscr{I}_{0}^{+}$. One thus has a contradiction.

Corollary 2. $N_{\mathscr{G}}$ is a trivial $\mathbb{S}^{2}$ bundle over $\mathscr{C} . N_{+}$is a topological $\mathbb{R}^{2}$ bundle over $\mathscr{I}^{+}$ such that $N_{+} \mid \Sigma \simeq T \mathbb{S}^{2}$ for every smooth slice $\Sigma \approx \mathbb{S}^{2}$ of $\mathscr{I}^{+}$, and is therefore non-trivial.

Proof. The 3-manifold $\mathscr{C}$, being orientable, is parallelizable. A parallelization of $\mathscr{C}$ together with a unit timelike vector field on $\tilde{M}$ define a parallelization of $\tilde{M}$. Hence $T \tilde{M}$ is trivial. It follows that $N_{\mathscr{G}}$ is trivial.

Let $\Sigma \approx \mathbb{S}^{2}$ be a smooth slice of $\mathscr{I}^{+}$. It is not difficult to see that $N_{+} \mid \Sigma$ is equivalent to a topological bundle over $\Sigma$ having the fibre over each $p \in \Sigma$ an open 2-cell neighbourhood of $p$ in $\Sigma$. This bundle is equivalent to $T \Sigma \simeq T \mathbb{S}^{2}$. Since $\mathbb{S}^{2}$ is not parallelizable, $T \mathbb{S}^{2}$ is non-trivial. Therefore $N_{+} \mid \Sigma$ is non-trivial and so is $N_{+}$.

The bundle equivalence class of any bundle is determined by the bundle it induces over a strong deformation retract of its base. Thus Corollary 2 determines the bundle equivalence classes of both $N_{\mathscr{C}}$ and $N_{+}$.

Remark 5.13. Suppose strong causality holds at $\mathscr{I}^{+}$, and $\mathscr{C}$ is homeomorphic to $\mathbb{R}^{3}$ (e.g. Minkowski space) One then has $\mathscr{I}^{+} \approx_{\text {diff }} \mathbb{S}^{2} \times \mathbb{R}, N_{\mathscr{G}} \approx_{\text {diff }} \mathbb{R}^{3} \times \mathbb{S}^{2}$ and $N_{+} \approx T \mathbb{S}^{2} \times \mathbb{R}$, and the homeomorphism $N_{+} \approx N_{\mathscr{C}}$ demonstrates the well-known result $T \mathbb{S}^{2} \times \mathbb{R} \approx \mathbb{S}^{2} \times \mathbb{R}^{3}$ (Karoubi [13, p. 22]).

Suppose $\mathscr{C} \approx_{\text {diff }} \mathbb{R}^{3}$, as would be the case if the Poincare conjecture were true. Then $M \approx_{\text {diff }} \mathbb{R}^{3} \times \mathbb{R} \approx_{\text {diff }} \mathbb{R}^{4}$ and $\mathscr{I}^{+} \approx_{\text {diff }}\left(C^{3}-\{p t\}.\right)$ where, by Theorem 5.1, $C^{3}$ is a contractible open 3-manifold which embeds in $\mathbb{S}^{3}$. Recall that a contractible open 3-manifold is said to be a Whitehead manifold if its every compact subspace admits a topological embedding into $\mathbb{S}^{3}$. Thus, in the present case, $C^{3}$ must be a Whitehead manifold. A theorem of McMillan [14] shows that any such manifold may be expressed as a monotone union of a sequence of P.L. cubes-with-handles such that each is contained and deformable to a point in the interior of the next. The fact that the whole of $C^{3}$ embeds in $\mathbb{S}^{3}$ is a non-trival restriction since many Whitehead manifolds are known to admit no such embeddings [15].

If the Poincaré conjecture is false then Theorem 5.1 may not give $\mathrm{M} \approx_{\text {diff }} \mathbb{R}^{4}$. Thus it is conceivable that there could exist a simple space-time with an underlying manifold diffeomorphic to an exotic $\mathbb{R}^{4}$.

In the case that the space-time admits an $\mathbb{R}^{3}$ Cauchy surface, one may use the following result to obtain a more useful description of the topology of $\mathscr{I}^{+}$.

Lemma 5.14. For any 3-manifold $N$, the following are equivalent:

(I) $N$ is homeomorphic to the complement of a point in a contractible open 3-manifold which embeds in $\mathbb{R}^{3}$;

(II) $N$ is homeomorphic to the complement in $\mathbb{R}^{3}$ of the intersection of a sequence of P.L. cubes-with-handles such that each is contained and deformable to a point in the interior of its predecessor. 
Proof. The result will be established as a corollary to the following.

Lemma 5.15. Let $T_{a}, T_{b}$ be P.L. cubes-with-handles in $\mathbb{S}^{3}$ such that $T_{a} \subset \stackrel{\circ}{T}_{b}$. Then the closed complements of $T_{a}$ and $T_{b}$ in $\mathbb{S}^{3}$ are P.L. cubes-with-handles $T_{a}^{\prime}, T_{b}^{\prime}$ such that $T_{b}^{\prime} \subset \stackrel{\circ}{T}_{a}^{\prime}$. Moreover if $T_{a}$ is deformable to a point in $\stackrel{\circ}{T}_{b}$, then $T_{b}^{\prime}$ is deformable to a point in $\stackrel{\circ}{T}_{a}^{\prime}$.

Proof. Any tamely embedded 2-sphere in $T_{a}^{\prime}$ divides $\mathbb{S}^{3}$ into a pair of 3-discs, one of which must be contained in $T_{a}^{\prime}$. Thus $T_{a}^{\prime}$ is irreducible. The Loop Theorem shows how a finite number of disjoint P.L. 1-handles may be removed from $T_{a}^{\prime}$ to yield a compact connected P.L. 3-submanifold-with-boundary $X$ of $T_{a}^{\prime}$ such that $\pi_{1}(\partial X) \rightarrow \pi_{1}(X)$ is trivial. The homotopy-homology ladder for $(X, \partial X)$ implies that $H_{1}(\partial X) \rightarrow H_{1}(X)$ is trivial and that $H_{1}(X) \rightarrow H_{1}(X, \partial X)$ is an isomorphism. The Mayer-Vietoris sequence for $\left(\mathbb{S}^{3}, X, X^{\prime}\right)$, where $X^{\prime}$ is the closed complement of $X$ in $\mathbb{S}^{3}$, now gives $H_{1}(X) \cong 0$. Hence $H_{1}(X, \partial X) \cong 0$. And since Lefschetz duality gives $H_{2}(X, \partial X) \cong H^{1}(X) \cong H_{1}(X) \cong 0$, the homology sequence for $(X, \partial X)$ implies $H_{1}(\partial X) \cong 0$. Thus $\partial X$ is a disjoint union of P.L. embedded 2-spheres, $X$ is a 3-disc and $T_{a}^{\prime}$ is a cube-with-handles. Similarly $T_{b}^{\prime}$ is a cube-with-handles. Since $T_{a}$ and $T_{b}$ are P.L. so are $T_{a}^{\prime}$ and $T_{b}^{\prime}$. The inclusion $T_{b}^{\prime} \subset \stackrel{\circ}{T}_{a}^{\prime}$ is obvious.

Each of $T_{a}, T_{a}^{\prime}, T_{b}$ and $T_{b}^{\prime}$ has the homotopy type of a finite wedge of circles, and so has a free, finitely generated fundamental group, with trivial homology and homotopy groups in dimensions greater than 1. By the Mayer-Vietoris sequence for $\left(\mathbb{S}^{3}, T_{a}, T_{a}^{\prime}\right)$ one has $\chi\left(T_{a}\right)+\chi\left(T_{a}^{\prime}\right)=\chi\left(\dot{T}_{a}\right)=2 \chi\left(T_{a}\right)$. Hence $H_{*}\left(T_{a}^{\prime}\right) \cong H_{*}\left(T_{a}\right)$. By hypothesis, the inclusion $j: T_{a} \rightarrow T_{b}$ is homotopic in $T_{b}$ to a constant map and so induces a trivial homomorphism of graded homology modules. The reduced homology sequence for the pair $\left(T_{b}, T_{a}\right)$ therefore gives $H_{1}\left(T_{b}, T_{a}\right) \cong H_{1}\left(T_{b}\right)$ and $H_{2}\left(T_{b}, T_{a}\right) \cong H_{1}\left(T_{a}\right)$. Consider the reduced homology sequence for the pair $\left(T_{a}^{\prime}, T_{b}^{\prime}\right)$ :

$$
\rightarrow H_{1}\left(T_{b}^{\prime}\right) \rightarrow H_{1}\left(T_{a}^{\prime}\right) \rightarrow H_{1}\left(T_{a}^{\prime}, T_{b}^{\prime}\right) \rightarrow 0 .
$$

By duality and the universal coefficient theorem one has $H_{1}\left(T_{a}^{\prime}, T_{b}^{\prime}\right) \cong H_{1}\left(\mathbb{S}^{3}-T_{a}\right.$, $\left.\mathbb{S}^{3}-T_{b}\right) \cong H^{2}\left(T_{b}, T_{a}\right) \cong H_{2}\left(T_{b}, T_{a}\right)$ since both $H_{2}\left(T_{b}, T_{a}\right) \cong H_{1}\left(T_{a}\right)$ and $H_{1}\left(T_{b}, T_{a}\right) \cong$ $H_{1}\left(T_{b}\right)$ are free and finitely generated. Hence $H_{1}\left(T_{a}^{\prime}, T_{b}^{\prime}\right) \cong H_{1}\left(T_{a}\right) \cong H_{1}\left(T_{a}^{\prime}\right)$. But any epimorphism of finitely generated free groups of equal rank is necessarily an isomorphism. Thus the inclusion $j^{\prime}: T_{b}^{\prime} \rightarrow T_{a}^{\prime}$ induces a trivial homomorphism of 1-dimensional homology modules. Since $\pi_{1}\left(T_{b}^{\prime}\right)$ and $\pi_{1}\left(T_{a}^{\prime}\right)$ are both free, the homomorphisms $\pi_{1}\left(T_{b}^{\prime}\right) \rightarrow H_{1}\left(T_{b}^{\prime}\right)$ and $\pi_{1}\left(T_{a}^{\prime}\right) \rightarrow H_{1}\left(T_{a}^{\prime}\right)$ are both isomorphisms, and the homotopy-homology ladder for the pair $\left(T_{a}^{\prime}, T_{b}^{\prime}\right)$ gives that $j^{\prime}$ induces a trivial homomorphism of fundamental groups. But $T_{b}^{\prime}$ has the homotopy-type of a wedge of circles, and so $j^{\prime}: T_{b}^{\prime} \rightarrow T_{a}^{\prime}$ must be homotopic in $T_{a}^{\prime}$ to a constant map. Since $T_{a}^{\prime}$ is P.L., any such homotopy may be deformed so as to avoid $\dot{T}_{a}^{\prime}$. Thus $T_{b}^{\prime}$ is deformable in $\stackrel{\circ}{T}_{a}^{\prime}$ to a point.

Suppose that (I) holds. The contractible open 3-manifold is a Whitehead manifold $W$ which one may assume to be realised as an embedded submanifold of $\mathbb{S}^{3}$. A previously quoted therorem of McMillan gives that $W$ is the union of a sequence of P.L. cubes-with-handles $T_{i}$ in $\mathbb{S}^{3}$ such that each $T_{i}$ is contained and deformable to a point in $\stackrel{\circ}{T}_{i+1}$. For each $i$ let $T_{i}^{\prime}$ be the closed complement of $T_{i}$ 
in $\mathbb{S}^{3}$. One then has $N \approx W-\{p t\}=.\bigcup_{i} T_{i}-\{p t\}=.\left(\mathbb{S}^{3}-\{p t\}.\right)-\bigcap_{i} T_{i}^{\prime}=\mathbb{R}^{3}-$ $\bigcap_{i} T_{i}^{\prime}$ under the identification $\mathbb{R}^{3}=\mathbb{S}^{3}-\{p t$.$\} . The lemma now implies (II).$

Suppose now that (II) holds. One then has $N=\mathbb{R}^{3}-\bigcap_{i} T_{i}$, where the $T_{i}$ are P.L. cubes-with-handles such that each $T_{i+1}$ is contained and deformable to a point in $\stackrel{\circ}{T}_{i}$. Let the one-point compactification of $\mathbb{R}^{3}$ be identified with $\mathbb{S}^{3}$, and for each $i$ let $T_{i}^{\prime}$ be the closed complement of $T_{i}$ in $\mathbb{S}^{3}$. One has $T_{i}^{\prime} \subset \stackrel{T}{T}_{i+1}^{\prime}$ for all $i$, and hence that $W:=\bigcup_{i} T_{i}^{\prime}$ is an open submanifold of $\mathbb{S}^{3}$. One also has $N \approx\left(\mathbb{S}^{3}-\{\infty\}\right)-\bigcap_{i} T_{i}=W-\{\infty\}$. For any $r \geqq 0$, a continuous image of $\mathbb{S}^{r}$ in $N$ is contained in $T_{i}^{\prime}$ for some $i$. Since the lemma gives that each $T_{i}^{\prime}$ is contained and deformable to a point in $\stackrel{\circ}{T}_{i+1}^{\prime}$, one must have $\pi_{r}(W) \cong 0$ for all $r \geqq 0$. Hence $W$ is contractible.

This shows that, for any simple space-time having an $\mathbb{R}^{3}$ Cauchy surface, the topology of $\mathscr{I}^{+}$may be realised as the complement in $\mathbb{R}^{3}$ of the intersection of a sequence of P.L. cubes-with-handles, each of which is contained and deformable to a point in the interior of its predecessor.

Subject to the truth of the Poincare conjecture, there has now emerged a general picture of an asymptotic null completion $(\tilde{M}, \tilde{\mathbf{g}})$ of a simple space-time $(M, \mathbf{g})$. Specifically, $\tilde{M}$ may be realised as an open dense submanifold-with-boundary of $\mathbb{S}^{3} \times[-1,1]$ such that

(a) $M=\left(\mathbb{S}^{3}-\{p t\}.\right) \times(-1,1)$;

(b) for each $t \in(-1,1)$ the set $\left(\mathbb{S}^{3}-\{p t\}.\right) \times\{t\}$ is a Cauchy surface of $(M, \mathbf{g})$;

(c) $\mathscr{I}^{+}$(respectively $\left.\mathscr{I}^{-}\right)$is the complement in $\left(\mathbb{S}^{3}-\{p t\}.\right) \times\{+1\}$ (respectively $\left.\left(\mathbb{S}^{3}-\{p t\}.\right) \times\{-1\}\right)$ of a monotone intersection of P.L. cubes-with-handles such that each is contained and deformable to a point in the interior of its predecessor;

Moreover spatial infinity, defined formally as the inverse limit of sets $\tilde{M}-I(\mathscr{K}, \tilde{M})$ for all compact sets $\mathscr{K}$ of $M$, is represented as the set $\{p t\} \times.[-1,1]$ identified to a point.

\section{Further Topological Considerations}

The previous sections give much information concerning the general properties of simple space-times and their asymptotic null completions. In particular, they show that $N_{+}$is the unique $\mathbb{R}^{2}$ bundle over $\mathscr{I}^{+} \approx C^{3}-\{p t$.$\} admitting a homotopy$ equivalence $i: \mathbb{S}^{2} \rightarrow \mathscr{I}^{+}$such that $i^{*} N_{+} \simeq T \mathbb{S}^{2}$, and that $N_{\mathscr{C}}$ is the unique trivial $\mathbb{S}^{2}$ bundle over $\mathscr{C} \approx_{\text {diff }} \widetilde{\mathcal{S}}^{3}-\{p t$.$\} . The proofs of these results have used only the$ homotopy equivalence of the total spaces of $N_{+}$and $N_{\mathscr{G}}$. However the causal structure of any simple space-time demands a homeomorphism $N_{+} \approx N_{\mathscr{\zeta}}$, and it is not immediately clear that this can exist other than in the special case $\mathscr{C} \approx_{\text {diff }} \mathbb{R}^{3}, \mathscr{I}^{+} \approx \mathbb{S}^{2} \times \mathbb{R}$, for which one has $N_{+} \approx T \mathbb{S}^{2} \times \mathbb{R} \approx_{\text {diff }} \mathbb{R}^{3} \times \mathbb{S}^{2} \approx_{\text {diff }} N_{\mathscr{C}}$. The two following self-contained topological results show that the necessary 
homeomorphism $N_{+} \approx N_{\mathscr{C}}$ does in fact occur for all topologies of $\mathscr{I}^{+}$and $\mathscr{C}$ admitted by Theorem 5.1 and its corollaries.

The first result is of relevance to $N_{\mathscr{b}}$.

Proposition 6.1. For any smooth homotopy 3-sphere $\tilde{\mathbb{S}}^{3}$ one has $\left(\tilde{\mathbb{S}}^{3}-\{p t\}.\right) \times$ $\mathbb{S}^{2} \approx_{\text {diff }} \mathbb{R}^{3} \times \mathbb{S}^{2}$.

Proof. Let $D_{1}, D_{2}$ be disjoint smoothly embedded 3-discs in $\widetilde{\mathbb{S}}^{3}$, and let $X:=$ $\widetilde{\mathbb{S}}^{3}-\left(\dot{D}_{1} \cup D_{2}\right)$. By excision one has $H_{r}\left(X, \partial D_{1}\right) \cong H_{r}\left(\tilde{\mathbb{S}}^{3}-\dot{D}_{2}, D_{1}\right) \cong \tilde{H}_{r}\left(\tilde{\mathbb{S}}^{3}-D_{2}\right)$ for all $r \geqq 0$, and by Lefschetz duality and excision one has $H_{r}\left(\tilde{\mathbb{S}}^{3}-D_{2}\right) \cong$ $H^{3-r}\left(\widetilde{\mathbb{S}}^{3}-D_{2}, \partial D_{2}\right) \cong H^{3-r}\left(\widetilde{S}^{3}, D_{2}\right) \cong \widetilde{H}^{3-r}\left(\widetilde{S}^{3}\right) \cong \tilde{H}^{3-r}\left(\mathbb{S}^{3}\right) \cong H_{r}(p t$. $)$ for all such $r$. Thus $H_{*}\left(X, \partial D_{1}\right) \cong 0$. The simple connectivity of $\widetilde{\mathbb{S}}^{3}$ implies that $X$, which has the homotopy type of $\widetilde{\mathbb{S}}^{3}-\{t w o p t s$. $\}$, is simply connected. Since $\partial D_{1} \approx \mathbb{S}^{2}$ is also simply connected, one may apply the relative Hurewicz isomorphism theorem to obtain $\pi_{r}\left(X, \partial D_{1}\right) \cong 0$ for all $r \geqq 0$. It follows that $\partial D_{1}$ is a strong deformation retract of $X$. Similarly $\partial D_{2}$ is a strong deformation retract of $X$, and $\operatorname{so}\left(X ; \partial D_{1}, \partial D_{2}\right)$ is a smooth 3-dimensional $h$-cobordism.

Let $\pi: \widetilde{\mathbb{S}}^{3} \times \mathbb{S}^{2} \rightarrow \widetilde{\mathbb{S}}^{3}$ be the projection onto the first factor. Let $\hat{X}:=\pi^{-1}$ $\left(\widetilde{\mathbb{S}}^{3}-\left(\dot{D}_{1} \cup \dot{D}_{2}\right)\right), Y_{1}:=\pi^{-1}\left(D_{1}\right)$ and $Y_{2}:=\pi^{-1}\left(D_{2}\right)$. Then $\left(\hat{X} ; \partial Y_{1}, \partial Y_{2}\right)$ is a smooth 5-dimensional $h$-cobordism. The simple connectivity of $\partial Y_{1} \approx \partial Y_{2} \approx \mathbb{S}^{2} \times \mathbb{S}^{2}$ permits the application of the five-dimensional proper $h$-cobordism theorem of Freedman [12] to obtain $\hat{X} \approx \partial Y_{1} \times[0,1]$, and consequently $\hat{X} \cup Y_{1} \approx Y_{1}$. Since one clearly has $\widetilde{\mathbb{S}}^{3}-\{p t.\} \approx \widetilde{\mathbb{S}}^{3}-D_{2} \approx\left(X \cup D_{1}\right)^{\circ}$, there follows $\left(\widetilde{\mathbb{S}}^{3}-\{p t\}.\right) \times \mathbb{S}^{2} \approx$ $\pi^{-1}\left(\left(X \cup D_{1}\right)^{\circ}\right)=\left(\hat{X} \cup Y_{1}\right)^{\circ} \approx \stackrel{\circ}{Y}_{1} \approx \stackrel{\circ}{D}_{1} \times \mathbb{S}^{2} \approx \mathbb{R}^{3} \times \mathbb{S}^{2}$.

By Theorem 10.1 of [16, Essay IV], the concordance classes of DIFF structures on $\mathbb{R}^{3} \times \mathbb{S}^{2}$ are classified $\left[\mathbb{R}^{3} \times \mathbb{S}^{2}\right.$, TOP/DIFF $]$, or equivalently by $\pi_{2}(\mathrm{TOP} / \mathrm{DIFF}) \cong 0$. Thus all DIFF structures on $\mathbb{R}^{3} \times \mathbb{S}^{2}$ are concordant and hence isotopic. It follows that any homeomorphism of $\left(\widetilde{\mathbb{S}}^{3}-\{p t\}.\right) \times \mathbb{S}^{2}$ onto $\mathbb{R}^{3} \times \mathbb{S}^{2}$ may be composed with a homeomorphism of $\mathbb{R}^{3} \times \mathbb{S}^{2}$ onto itself to yield a diffeomorphism of $\left(\widetilde{\mathbb{S}}^{3}-\{p t\}.\right) \times \mathbb{S}^{2}$ onto $\mathbb{R}^{3} \times \mathbb{S}^{2}$.

It is now clear that, for a smooth Cauchy surface $\mathscr{C}$ of any topology admitted by Theorem 5.1, one has $N_{\mathscr{6}} \approx_{\text {diff }} \mathbb{R}^{3} \times \mathbb{S}^{2}$.

The next result is of relevance to the bundle $N_{+}$. Since the metric $\tilde{\mathbf{g}}$ has only been assumed to be $C^{0}, N_{+}$may only be $C^{0}$ and so it is necessary to work in the topological category.

Proposition 6.2. Let $C^{3}$ be a contractible open topological 3-manifold. Let $\pi: E \rightarrow C^{3}-\{p t$.$\} be a topological \mathbb{R}^{2}$-bundle admitting a homotopy equivalence $h: \mathbb{S}^{2} \rightarrow C^{3}-\{p t$.$\} such that h^{*} E \simeq T \mathbb{S}^{2}$. Then $E \approx \mathbb{R}^{3} \times \mathbb{S}^{2}$.

Proof. The topological manifold $C^{3}$, being 3-dimensional, admits a P.L. structure. Let $B^{3}$ be a regular neighbourhood of $p t$. in $C^{3}$ and let $\Sigma:=\dot{B}^{3}$. Then $\Sigma$ is a strong deformation retract of $C^{3}-\{p t$.$\} , and so h: \mathbb{S}^{2} \rightarrow C^{3}-\{p t$.$\} is homotopic in$ $C^{3}-\{p t$.$\} to a continuous map h_{\Sigma}: \mathbb{S}^{2} \rightarrow \Sigma \subset C^{3}-\{p t$. $\}$. Since $h$ is a homotopy equivalence, so is $h_{\Sigma}$. The Brouwer degree theorem (Spanier [4, p. 398]) therefore gives that $h_{\Sigma}: \mathbb{S}^{2} \rightarrow \Sigma \approx \mathbb{S}^{2}$ is homotopic to a homeomorphism $H_{\Sigma}: \mathbb{S}^{2} \rightarrow \Sigma \approx \mathbb{S}^{2}$. There follows $E \mid \Sigma \simeq H_{\Sigma}^{*} E \simeq h_{\Sigma}^{*} E \simeq h^{*} E \simeq T \mathbb{S}^{2}$. 
By Theorem 1.1 of [16, Essay II] there exists a P.L. structure on $E$ such that the mapping $\pi: E \rightarrow C^{3}-\{p t$. $\}$ is P.L. (The required, continuous $\mathbb{R}$-valued function on $E$ may be taken to be the logarithm of fibrewise distance, with respect to a complete topological metric on $E$, from a fixed section of $E$.) Let $C^{3}-\{p t$. $\}$ be identified with the image of a P.L. section of $E$. Then $\Sigma$ is a strong deformation retract of $E$, and $H_{\Sigma}: \mathbb{S}^{2} \rightarrow \Sigma$ may be regarded as a P.L. embedding of $\mathbb{S}^{2}$ into $E$. Let $\pi_{D}: E_{D} \rightarrow C^{3}-\{p t$. $\}$ be a 2-disc P.L. sub-bundle of $E$ such that $C^{3}-\{p t.\} \subset E_{D}$. Let $N^{3} \approx \Sigma \times[-1,1]$ be a regular neighbourhood of $\Sigma$ in $C^{3}-\{p t$. $\}$. Then $N:=\pi_{D}^{-1}\left(N^{3}\right)$ is a regular neighbourhood of $\Sigma$ in $E$. Since $\Sigma$ is a strong deformation retract of $N$, as well as of $E$, the homotopy sequence for the triple $(E, N, \Sigma)$ gives that the pair $(E, N)$ is $k$-connected for all $k \geqq 0$. One also has $N \approx \pi^{-1}(\Sigma) \times(-1,1) \approx T \mathbb{S}^{2} \times \mathbb{R} \approx \mathbb{R}^{3} \times \mathbb{S}^{2}$.

Lemma 6.3. For any compact subset $A$ of $E$ there exists a regular neighbourhood $N_{A} \approx N$ of $\Sigma$ such that $A \subset \stackrel{\circ}{N}_{A}$.

Proof. Let $A$ be any compact subset of $E$. Then $\pi(A)$ is a compact subset of $C^{3}-\{p t$. $\}$ and is contained in some compact P.L. subspace $A_{0}$ of $C^{3}-\{p t$. $\}$. By Lemma 3.7 of Hudson [17], the P.L. manifold $\mathrm{C}^{3}-\{p t$.$\} admits a triangulation$ by a locally finite simplical complex $K$ which contains a subcomplex $K_{0}$ triangulating $A_{0}$. If $\operatorname{dim}\left(K_{0}\right)=3$, then a finite sequence of 3-dimensional elementary simplicial collapses yields a 2-dimensional subcomplex $K^{\prime}$ of $K_{0}$ such that $K_{0} \searrow^{s} K^{\prime}$. Hence, in general, $K_{0}$ admits a subcomplex $K_{1}$, $\operatorname{dim}\left(K_{1}\right) \leqq 2=\operatorname{dim}(E)-3$, such that $K_{0} \searrow^{s} K_{1}$. The Engulfing Theorem 7.4 of [17] gives that there exists a P.L. homeomorphism $h_{1}: E \rightarrow E$ such that $\left|K_{1}\right| \subset h_{1}(N)$, whereby Lemma 7.1 of [17] gives that there exists a P.L. homeomorphism $h_{2}: E \rightarrow E$ such that $\left|K_{0}\right| \subset h_{2} \circ h_{1}(N)$. There clearly exists a fibre preserving P.L. homeomorphism $h_{3}: E \rightarrow E$ throwing $A$ into the open neighbourhood $h_{2}{ }^{\circ} h_{1}(N)$ of $A_{0}=\left|K_{0}\right|$ in $E$. The regular neighbour$\operatorname{hood} N_{A}:=h_{3}^{-1} \circ h_{2} \circ h_{1}(N)$ of $\Sigma$ in $E$ is such that $A_{0} \subset N_{A}^{\circ}$.

Let $\mathcal{O} \subset N$ be a regular neighbourhood of $\Sigma$ in $E$. Then the Generalized Annulus Theorem 2.16 .2 of [17] gives $\stackrel{\circ}{N}-\dot{\mathcal{O}} \approx \dot{\mathcal{O}} \times[0,1)$. Lemma 6.3 implies that $E$ is covered by a sequence of regular neighbourhoods $N_{i} \approx N$ of $\Sigma$ such that $N_{i} \subset N_{i+1}$ for all $i$. Without loss of generality, assume $N_{1}=\mathcal{O}$. For each $i$, the Generalised Annulus Theorem gives $N_{i+1}-\dot{N}_{i} \approx \dot{N}_{i} \times[i, i+1]$. Hence $E-\dot{\mathcal{O}} \approx \dot{\mathcal{O}} \times[0, \infty) \approx$ $\dot{0} \times[0,1) \approx N-\dot{\mathcal{O}}$. It follows that there exists a homeomorphism of $E$ onto $\dot{N}$ leaving $\mathcal{O}$ fixed. Thus $E \approx N \approx \mathbb{R}^{3} \times \mathbb{S}^{2}$.

It is now clear that, for all topologies of $\mathscr{I}^{+}$and $\mathscr{C}$ admitted by Theorem 5.1, the total spaces of the corresponding, uniquely determined bundles $N_{+}$and $N_{\mathscr{C}}$ are homeomorphic to $\mathbb{R}^{3} \times \mathbb{S}^{2}$, and so are homeomorphic to one another.

\section{General Slices}

Theorem 5.1 gives that all slices of $\mathscr{I}^{+}$are compact and connected. This information may be used to generalize the two results of Sect. 4 established only for good slices of $\mathscr{I}^{+}$, namely Proposition 4.13(III) and Lemma 4.15, to apply to all slices.

Proposition 7.1. Every generator of $\mathscr{I}_{0}^{+}$cuts every slice of $\mathscr{I}^{+}$. 
Proof. Let $\Sigma$ be a slice of $\mathscr{I}^{+}$and let $\Sigma_{g}$ be a good slice of $\mathscr{I}^{+}$. By Proposition 4.13(III) every generator of $\mathscr{I}_{0}^{+}$which cuts $\Sigma$ must cut $\Sigma_{g}$. These generators thus define a homeomorphism of $\Sigma$ onto an open submanifold of $\Sigma_{g}$. Since $\Sigma$ is compact and $\Sigma_{g} \approx \mathbb{S}^{2}$ is connected, this homeomorphism is onto $\Sigma_{g}$. Hence every generator of $\mathscr{I}_{0}^{+}$which cuts $\Sigma_{g}$ must cut $\Sigma$. But Proposition 4.13(III) gives that every generator of $\mathscr{I}_{0}^{+}$cuts $\Sigma_{g}$. Hence every generator of $\mathscr{I}_{0}^{+}$cuts $\Sigma$.

Proposition 7.2. If $\Sigma$ is a slice of $\mathscr{I}^{+}$then $\mathscr{I}^{-} \subset I^{-}(\Sigma, \tilde{M})$.

Proof. Since $\Sigma$ is compact there exists a compact set $\mathscr{K}$ of $M$ such that $\Sigma \subset I^{+}(\mathscr{K}, \tilde{M})$. Consider the good slice $\Sigma_{g}:=\dot{I}^{+}(\mathscr{K}, \tilde{M}) \cap \mathscr{I}^{+}$of $\mathscr{I}^{+}$. Let $p \in \Sigma_{g}$. By Proposition 7.1 the generator of $\mathscr{I}^{+}$through $p$ cuts $\Sigma$ at some point $q \in \Sigma$. One cannot have $p \in J^{+}(q, \tilde{M})$ for this would imply $p \in I^{+}(\mathscr{K}, \tilde{M})$ which is incompactible with $p \in \Sigma_{g} \subset \dot{I}^{+}(\mathscr{K}, \tilde{M})$. Hence $p \in J^{-}(q, \tilde{M}) \subset J^{-}(\Sigma, \tilde{M})$ and more generally $\Sigma_{g} \subset J^{-}(\Sigma, \tilde{M})$. By Lemma 4.15 one now has $\mathscr{I}^{-} \subset I^{-}\left(\Sigma_{g}, \tilde{M}\right) \subset I^{-}(\Sigma, \tilde{M})$.

These two results enable one to establish the following result concerning the causal relationship between a slice of $\mathscr{I}^{+}$and a Cauchy surface of $(M, \mathbf{g})$.

Proposition 7.3. If $\Sigma$ is a slice of $\mathscr{I}^{+}$, and $\mathscr{C}$ is a Cauchy surface for $(M, \mathbf{g})$, then $\mathscr{C}-I^{-}(\Sigma, \tilde{M})$ is compact.

Proof. Suppose $\mathscr{C}-I^{-}(\Sigma, \tilde{M})$ is non-compact. Then there exists a sequence of points $q_{i}$ therein without cluster point in $\tilde{M}$. For each $i$ there exists a timelike curve $\mu_{i}$ from $\mathscr{I}^{-} \subset I^{-}(\Sigma, \tilde{M})$ to $q_{i} \in \mathscr{C}-I^{-}(\Sigma, \tilde{M})$. Each $\mu_{i}$ admits a segment $v_{i}$ from some point $p_{i} \in \dot{I}^{-}(\Sigma, \tilde{M}) \cap J^{-}(\mathscr{C}, \tilde{M}) \subset\left(\tilde{M}-\mathscr{I}^{-}\right) \cap\left(\tilde{M}-\mathscr{I}^{+}\right)=M$ to $q_{i} \in \mathscr{C}$. Lemma 3.3(II) gives $\dot{I}^{-}(\Sigma, \tilde{M})-\mathscr{I}^{+}=\dot{J}^{-}(\Sigma, \tilde{M})-\mathscr{I}^{+}$, and Lemma 4.13(II) gives that $\dot{J}^{-}(\Sigma, \tilde{M})$ is compact. Since $J^{-}(\mathscr{C}, \tilde{M}) \subset \tilde{M}-\mathscr{I}^{+}$is closed in $\tilde{M}$, it follows that the $p_{i}$ admit a cluster point $p \in J^{-}(\Sigma, \tilde{M}) \cap J^{-}(\mathscr{C}, \tilde{M}) \subset M$. Since one has $\left|v_{i}\right| \subset J^{-}(\mathscr{C}, \tilde{M})$ for all $i$, the $v_{i}$ therefore admit a future endless causal cluster curve $v$ in $\tilde{M}$ from $p \in M$ such that $|v| \subset J^{-}(\mathscr{C}, \tilde{M})$. But by Lemma $4.2, v$ would have to cut $I^{+}(\mathscr{C}, \tilde{M})$. This gives a contradiction since $\mathscr{C}$ is acausal in $(\tilde{M}, \tilde{\mathbf{g}})$.

The following is the final result.

Theorem 7.4. Every non-empty locally acausal compact connected topological 2-submanifold of $\mathscr{I}^{+}$is acausal and contained in $\mathscr{I}_{0}^{+}$.

Proof. Since $(M, \mathbf{g})$ is globally hyperbolic, there exists a continuous surjection $\tau: M \rightarrow(-1,1)$ which is monotonically strictly increasing along every timelike curve of $(M, \mathbf{g})$ and whose level sets are Cauchy surfaces of $(M, \mathbf{g})$. For any $p \in \mathscr{I}^{+}$and any $\delta>0$, every neighbourhood $\mathscr{N}_{p}$ of $p$ in $\tilde{M}$ contains a neighbourhood $\mathscr{N}_{p}^{\prime}$ of $p$ in $\tilde{M}$ such that $\tau \mid \mathscr{N}_{p}^{\prime} \cap M>1-\delta$.

Lemma 7.5. No non-empty locally acausal compact connected topological 2-submanifold of $\mathscr{I}^{+}$bounds a compact topological 3-submanifold-with-boundary of $\mathscr{I}^{+}$.

Proof. Suppose, to the contrary, that there exists a compact topological 3-submanifold-with-boundary $\mathscr{X}$ of $\mathscr{I}^{+}$such that $\partial \mathscr{X}$ is non-empty connected and locally acausal. Let the inclusion of $\mathscr{X}:=\mathscr{X} \times\{0\}$ into $\tilde{M}$ be extended to a topological embedding of $\mathscr{X} \times[0,1]$ into $\tilde{M}$ such that $\mathscr{X} \times(0,1] \subset M$, with 
$\partial \mathscr{X} \times[0,1]$ locally acausal. Let $t_{0} \in(-1,1)$ be the supremum of $\tau$ on the compact set $\mathscr{X} \times\{1\} \subset M$.

Suppose $\partial \mathscr{X}$ is a future boundary of $\mathscr{X}$. Then $\partial \mathscr{X} \times[0,1]$ is contained in the future boundary of $\mathscr{X} \times[0,1]$. Let $\gamma$ be a generator of $\mathscr{I}^{+}$which cuts $\partial \mathscr{X}$. Then $\gamma$ is totally past imprisoned by $\mathscr{X}$ and there exists $r \in L^{-}(\gamma, \tilde{M}) \subset \mathscr{X} \cap\left(\mathscr{I}^{+}-\mathscr{I}_{0}^{+}\right)$. Since $\mathscr{X}$ is compact and $\mathscr{I}^{+}$is non-compact, the open set $\tilde{M}-(\mathscr{X} \times[0,1])$ of $\tilde{M}$ must have non-empty intersection with $\mathscr{I}^{+}$and so must contain a point $p \in M$ such that $\tau(p)>t_{0}$. Since one has $p \in M \subset I^{-}(r, \tilde{M})$, there exists a timelike curve $\lambda$ of $(\tilde{M}, \tilde{\mathbf{g}})$ from $p \notin \mathscr{X} \times[0,1]$ to $r \in \mathscr{X} \times[0,1]$. Since $\partial \mathscr{X} \times[0,1]$ is contained in the future boundary of $\mathscr{X} \times[0,1], \lambda$ must cut $\partial(\mathscr{X} \times[0,1]) \cap M=(\partial \mathscr{X} \times(0,1]) \cup(\mathscr{X} \times\{1\})$ at some point $q \in \mathscr{X} \times\{1\} \subset M$. One then has $\tau(p)>t_{0} \geqq \tau(q)$ which is impossible since $\tau \circ \lambda$ is monotonically strictly increasing.

Now suppose $\partial \mathscr{X}$ is a past boundary of $\mathscr{X}$. Then $\partial \mathscr{X} \times[0,1]$ is contained in the past boundary of $\mathscr{X} \times[0,1]$. Let $\gamma^{\prime}$ be a generator of $\mathscr{I}^{+}$which cuts $\partial \mathscr{X}$. Then the compact set $\mathscr{X}$ must totally future imprison $\gamma^{\prime}$ and so have non-empty intersection with $\mathscr{I}^{+}-\mathscr{I}_{0}^{+}$. Thus $\mathscr{I}^{+}-\mathscr{I}_{0}^{+}$is non-empty and moreover, being closed with no compact component, cannot be contained by $\mathscr{X}$. Hence there exists $r^{\prime} \in\left(\mathscr{I}^{+}-\mathscr{I}_{0}^{+}\right)-\mathscr{X}$. Choose any $p^{\prime} \in \mathscr{X} \times(0,1] \subset M$ such that $\tau\left(p^{\prime}\right)>t_{0}$. Since one has $M \subset I^{-}\left(r^{\prime}, \tilde{M}\right)$, there exists a timelike curve $\lambda^{\prime}$ of $(\tilde{M}, \tilde{\mathbf{g}})$ from $p^{\prime} \in \mathscr{X} \times[0,1]$ to $r^{\prime} \notin \mathscr{X} \times[0,1]$. Since $\partial \mathscr{X} \times[0,1]$ is contained in the past boundary of $\mathscr{X} \times[0,1], \lambda^{\prime}$ must cut $\partial(\mathscr{X} \times[0,1]) \cap M=(\partial \mathscr{X} \times(0,1]) \cup(\mathscr{X} \times\{1\})$ at some point $q^{\prime} \in \mathscr{X} \times\{1\} \subset M$. Since $\tau \circ \lambda^{\prime}$ is monotonically strictly increasing there follows $\tau\left(p^{\prime}\right)<\tau\left(q^{\prime}\right) \leqq t_{0}$, which gives a contradiction.

Let $\Sigma$ be a non-empty locally acausal compact connected topological 2-submanifold of $\mathscr{I}^{+}$. Let $\mathscr{K} \subset M$ be a compact set such that $\Sigma \subset I^{+}(\mathscr{K}, \tilde{M})$. Then the $\operatorname{good}$ slice $\Sigma_{0}:=\dot{I}^{+}(\mathscr{K}, \tilde{M}) \cap \mathscr{I}^{+} \subset \mathscr{I}_{0}^{+}$of $\mathscr{I}^{+}$does not intersect $\Sigma$.

For the purposes of the next two lemmas, let $\mathscr{I}^{+}$be identified with $C^{3}-\{p t$.$\} ,$ where $C^{3}$ is a contractible open 3-manifold.

Lemma 7.6. $C^{3}$ admits compact topological 3-submanifolds-with-boundary $\mathscr{Y}$ and $\mathscr{Y}_{0}$ which are bounded by $\Sigma$ and $\Sigma_{0}$ respectively, and such that pt. $\in \dot{\mathscr{Y}} \cap \mathscr{Y}_{0}$.

Proof. Let the inclusion of $\Sigma:=\Sigma \times\{0\}$ into $C^{3}$ be extended to a topological embedding of $\Sigma_{\mathrm{th}}:=\Sigma \times[-1,1]$ into $C^{3}$. Then $C^{3}-\Sigma_{\mathrm{th}}^{\circ}$ is a 3-submanifold-withboundary of $C^{3}$. Since $C^{3}$ is connected, each component of $C^{3}-\Sigma_{\text {th }}^{\circ}$ is bounded by a non-empty union of components of $\partial \Sigma_{\mathrm{th}}$. Moreover, an elementary MayerVietoris argument gives that $C^{3}-\Sigma_{\text {th }}^{\circ}$ has precisely two components. Each of the two components of $\partial \Sigma_{\mathrm{th}}=(\Sigma \times\{-1\}) \cup(\Sigma \times\{1\})$ must therefore bound a component of $C^{3}-\Sigma_{\mathrm{th}}^{\circ}$. The homology sequence for the pair $\left(C^{3}, \Sigma_{\mathrm{th}}^{\circ}\right)$ gives

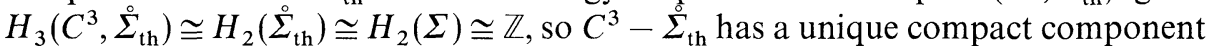
$\mathscr{Y}^{\#}$. Without loss of generality, assume $\partial \mathscr{Y}^{\#}=\Sigma \times\{1\}$. Then $\mathscr{Y}:=\mathscr{Y}^{\#} \cup(\Sigma \times[0,1])$ is a compact topological 3-submanifold-with-boundary of $C^{3}$ such that $\partial \mathscr{Y}=\Sigma \times$ $\{0\}=\Sigma$. The use of Lemma 7.5 gives $p t . \in \mathscr{Y}$, and one clearly has $p t . £ \subset \mathscr{I}^{+}=$ $C^{3}-\{p t$.$\} . Hence p t . \in \mathscr{Y}-\Sigma=\mathscr{Y}$. The proof for $\Sigma_{0}$ is similar.

Lemma 7.7. There exists a compact topological 3-submanifold-with-boundary $\mathscr{Z}$ of $C^{3}-\{p t$.$\} such that \partial \mathscr{Z}=\Sigma \cup \Sigma_{0}$. 
Proof. Both $\mathscr{Y}$ and $\mathscr{Y}_{0}$ are non-empty compact topological 3-submanifolds-withboundary of the connected non-compact 3-manifold $C^{3}$. Since $\partial \mathscr{Y}=\Sigma$ and $\partial \mathscr{Y}_{0}=\Sigma_{0}$ do not intersect, one has that $\mathscr{Y} \cup \mathscr{Y}_{0}$ and $\mathscr{Y} \cap \mathscr{Y}_{0}$, the latter being non-empty by Lemma 7.6, are compact topological 3-submanifolds-with-boundary of $C^{3}$ such that each is bounded by a non-empty union of components of $\Sigma \cup \Sigma_{0}$. Again since $\partial \mathscr{Y}=\Sigma$ and $\partial \mathscr{Y}_{0}=\Sigma_{0}$ do not intersect, one has $\mathscr{Y} \cap \mathscr{Y}_{0} \subset\left(\mathscr{Y} \cup \mathscr{Y}_{0}\right)^{\circ}$, and hence that $\partial\left(\mathscr{Y} \cup \mathscr{Y}_{0}\right)$ and $\partial\left(\mathscr{Y} \cap \mathscr{Y}_{0}\right)$ are disjoint components of $\Sigma \cup \Sigma_{0}$. Thus $\mathscr{Z}:=\mathscr{Y} \cup \mathscr{Y}_{0}-\left(\mathscr{Y} \cap \mathscr{Y}_{0}\right)^{\circ}$ is a compact topological 3-submanifold-with-boundary of $C^{3}$ such that $\partial \mathscr{Z}=\partial\left(\mathscr{Y} \cup \mathscr{Y}_{0}\right) \cup \partial\left(\mathscr{Y} \cap \mathscr{Y}_{0}\right)=\Sigma \cup \Sigma_{0}$. By Lemma 7.6 one has

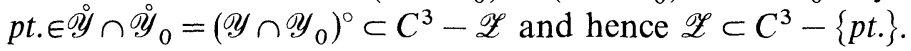

Lemma 7.8. $\mathscr{Z} \subset \mathscr{I}_{0}^{+}$.

Proof. Suppose, to the contrary, that there exists $r \in \mathscr{Z} \cap\left(\mathscr{I}^{+}-\mathscr{I}_{0}^{+}\right)$. Let the inclusion of $\mathscr{Z}:=\mathscr{Z} \times\{0\}$ into $\tilde{M}$ be extended to a topological embedding of $\mathscr{Z} \times[0,1]$ into $\tilde{M}$ such that $\mathscr{Z} \times(0,1] \subset M$, with $\partial \mathscr{Z} \times[0,1]=(\Sigma \times[0,1]) \cup$ $\left(\Sigma_{0} \times[0,1]\right)$ locally acausal, and moreover such that $\Sigma_{0} \times[0,1] \subset \dot{I}^{+}(\mathscr{K}, \tilde{M})$. Let $t_{0} \in(-1,1)$ be the supremum of $\tau: M \rightarrow(-1,1)$ on the compact set $\mathscr{Z} \times\{1\}$ of $M$.

Suppose $\Sigma_{0}$ was a future boundary component of $\mathscr{Z}$. Then $\mathscr{Z}-\Sigma_{0}$ would intersect $\mathscr{I}^{+}-I^{+}(\mathscr{K}, \tilde{M})$ but not $\Sigma_{0}=\dot{I}^{+}(\mathscr{K}, \tilde{M}) \cap \mathscr{I}^{+}$and so, being connected, would be a subset of $\mathscr{I}^{+}-I^{+}(\mathscr{K}, \tilde{M})$. One would thus have $r \in \Sigma \subset \mathscr{Z}-\Sigma_{0} \subset \tilde{M}-$ $I^{+}(\mathscr{K}, \tilde{M})$. However this is impossible since the inclusions $\mathscr{K} \subset M \subset I^{-}(r, \tilde{M})$ imply $r \in I^{+}(\mathscr{K}, \tilde{M})$. Thus $\Sigma_{0}$, being connected, is a past boundary component of $\mathscr{Z}$. Hence $\Sigma_{0} \times[0,1]$ is contained in the past boundary of $\mathscr{Z} \times[0,1]$.

Suppose $\Sigma$ is a future boundary component of $\mathscr{Z}$. Then $\Sigma \times[0,1]$ is contained in the future boundary of $\mathscr{Z} \times[0,1]$. Lemma 4.14 gives $\mathscr{I}^{+}-\mathscr{I}_{0}^{+} \subset I^{+}(\mathscr{K}, \tilde{M})$. Moreover $\mathscr{I}^{+}-\mathscr{I}_{0}^{+}$is closed non-empty and non-compact, and so cannot be contained in the compact set $\mathscr{Z} \times[0,1]$. It follows that the open set $I^{+}(\mathscr{K}, \tilde{M})-$ $(\mathscr{Z} \times[0,1])$ of $\tilde{M}$ has non-empty intersection with $\mathscr{I}^{+}-\mathscr{I}_{0}^{+}$and so contains a point $p \in M$ such that $\tau(p)>t_{0}$. Since one has $M \subset I^{-}(r, \tilde{M})$, there exists a timelike curve $\lambda$ of $(\tilde{M}, \tilde{\mathbf{g}})$ from $p \in I^{+}(\mathscr{K}, \tilde{M})-(\mathscr{Z} \times[0,1])$ to $r \in \mathscr{Z} \times[0,1]$. Clearly $|\lambda| \subset I^{+}(\mathscr{K}, \tilde{M})$, so $\lambda$ cannot cut $\Sigma_{0} \times[0,1] \subset i^{+}(\mathscr{K}, \tilde{M})$. Since $\Sigma \times[0,1]$ is contained in the future boundary of $\mathscr{Z} \times[0,1], \lambda$ must therefore cut $\partial(\mathscr{Z} \times[0,1]) \cap$ $M=(\Sigma \times(0,1]) \cup\left(\Sigma_{0} \times(0,1]\right) \cup(\mathscr{Z} \times\{1\})$ at some point $q \in \mathscr{Z} \times\{1\} \subset M$. But then one has $\tau(p)>t_{0} \geqq \tau(q)$ which is impossible since $\tau \circ \lambda$ is monotonically strictly increasing.

Now suppose $\Sigma$ is a past boundary component of $\mathscr{Z}$. Then $\Sigma \times[0,1]$ is contained in the past boundary of $\mathscr{Z} \times[0,1]$. Since the compact set $\mathscr{Z}$ cannot contain all of the closed non-empty non-compact set $\mathscr{I}^{+}-\mathscr{I}_{0}^{+}$, there exists $r^{\prime} \in\left(\mathscr{I}^{+}-\mathscr{I}_{0}^{+}\right)-\mathscr{Z}$. Choose any $p^{\prime} \in \mathscr{Z} \times(0,1] \subset M$ such that $\tau\left(p^{\prime}\right)>t_{0}$. Since one has $M \subset I^{-}\left(r^{\prime}, \tilde{M}\right)$ there exists a timelike curve $\lambda^{\prime}$ of $(\tilde{M}, \tilde{\mathbf{g}})$ from $p^{\prime} \in \mathscr{Z} \times[0,1]$ to $r^{\prime} \notin \mathscr{Z} \times[0,1]$. Since $\Sigma \times[0,1]$ and $\Sigma_{0} \times[0,1]$ are both contained in the past boundary of $\mathscr{Z} \times[0,1], \lambda^{\prime}$ must cut $\partial(\mathscr{Z} \times[0,1]) \cap M=(\Sigma \times(0,1]) \cup\left(\Sigma_{0} \times(0,1]\right) \cup$ $(\mathscr{Z} \times\{1\})$ at some point $q^{\prime} \in \mathscr{Z} \times\{1\} \subset M$. Since $\tau \circ \lambda^{\prime}$ is monotonically strictly increasing there follows $\tau\left(p^{\prime}\right)<\tau\left(q^{\prime}\right) \leqq t_{0}$, which gives a contradiction.

Lemma 7.9. $\Sigma$ is acausal.

Proof. If $\Sigma$ and $\Sigma_{0}$ were both future boundary components of $\mathscr{Z} \subset \mathscr{I}_{0}^{+}$, any 
generator $\gamma$ of $\mathscr{I}^{+}$which cut $\partial \mathscr{Z}=\Sigma \cup \Sigma_{0}$ would be totally future imprisoned by $\mathscr{Z}$, with $L^{+}(\gamma, \tilde{M})$ a non-empty subset of $\mathscr{Z} \cap\left(\mathscr{I}^{+}-\mathscr{I}_{0}^{+}\right)$. This contradicts Lemma 7.8. Similarly $\Sigma$ and $\Sigma_{0}$ cannot both be past boundary components of $\mathscr{Z}$.

Suppose $\Sigma \subset \mathscr{I}_{0}^{+}$is not acausal. Then there exists a non-degenerate generating segment $\kappa$ of $\mathscr{I}_{0}^{+}$from some $q \in \Sigma$ to some $s \in \Sigma$. Suppose $\Sigma$ and $\Sigma_{0}$ are respectively future and past boundary components of $\mathscr{Z}$. Then $\kappa$ must leave $\mathscr{Z}$ at $q \in \Sigma$ and, in order to reach $s \in \Sigma$, must enter $\mathscr{Z}$ through $\Sigma_{0}$. Hence $\kappa$ admits a segment $v$ from $q \in \Sigma$ to some $r \in \Sigma_{0}$. By Proposition 4.13(II), the past endless generating segment $\mu^{-}$of $\mathscr{I}_{0}^{+}$to $q$ is such that $L^{-}\left(\mu^{-}, \tilde{M}\right)=\varnothing$, and so cannot be totally past imprisoned by $\mathscr{Z}$. Thus $\mu^{-}$, being unable to enter $\mathscr{Z}$ through $\Sigma$, must admit a segment $\mu$ from some $p \in \Sigma_{0}$ to $q$. The concatenation of $\mu$ and $v$ is a non-degenerate causal curve from $p \in \Sigma_{0}$ to $r \in \Sigma_{0}$. This gives a contradiction since $\Sigma_{0}$ is a good slice of $\mathscr{I}^{+}$. A similar contradiction is obtained if $\Sigma$ and $\Sigma_{0}$ are respectively past and future boundary components of $\mathscr{Z}$.

The result now follows by Lemmas 7.8 and 7.9.

A slice of $\mathscr{I}^{+}$, according to the provisional Definition 4.11, is a compact topological 2-submanifold $\Sigma$ of $\mathscr{I}^{+}$which is contained in $\mathscr{I}_{0}^{+}$and acausal in $(\tilde{M}, \tilde{\mathbf{g}})$. It is now appropriate that this definition be reconsidered. Observe that, by Theorem 7.4, the hypothesis that $\Sigma$ be contained in $\mathscr{I}_{0}^{+}$is redundant. And by Theorem 5.1(IV), the possibility for disconnected slices cannot be realised. Moreover Theorem 7.4 shows that, if a connectivity hypothesis is explicitly imposed, then the hypothesis of acausality may be weakened to one of local acausality. One is thus led to redefine slices of $\mathscr{I}^{+}$in the following manner.

Definition 7.10. A non-empty locally acausal compact connected topological 2-submanifold of $\mathscr{I}^{+}$is a slice of $\mathscr{I}^{+}$. A slice of $\mathscr{I}^{+}$of the form $\dot{J}^{+}(\mathscr{K}, \tilde{M}) \cap \mathscr{I}^{+}$ for some compact set $\mathscr{K} \subset M$ is a good slice of $\mathscr{I}^{+}$.

Theorem 5.1(IV) and Theorem 7.4 together establish the equivalence of Definitions 4.11 and 7.10. All previous results concerning slices of $\mathscr{I}^{+}$are therefore unaffected. Theorem 7.4 may be re-expressed to the effect that a slice of $\mathscr{I}^{+}$, in the sense of Definition 7.10, is necessarily acausal and contained in $\mathscr{I}_{0}^{+}$.

\section{Concluding Remarks}

The fundamental causal and topological properties of simple space-times have been identified. Certain problems have, however, been left open. For example:

(1) Given any homotopy 3 -sphere $\widetilde{\mathbb{S}}^{3}$, and any pair of contractible open 3-manifolds $C_{+}^{3}, C_{-}^{3}$ which embed in $\widetilde{\mathbb{S}}^{3}$, does there exist a simple space-time with a Cauchy surface homeomorphic to $\widetilde{\mathbb{S}}^{3}-\{p t\},. \mathscr{I}^{+}$homeomorphic to $C_{+}^{3}-\{p t$. and $\mathscr{I}^{-}$homeomorphic to $C_{-}^{3}-\{p t$.$\} ?$

(2) Does strong causality violation at $\mathscr{I}^{+}$imply that $\mathscr{I}^{+}$has a topology different from $\mathbb{S}^{2} \times \mathbb{R}$ ?

(3) If $\left(\tilde{M}_{a}, \tilde{\mathbf{g}}_{a}\right)$ and $\left(\tilde{M}_{b}, \tilde{\mathbf{g}}_{b}\right)$ are two asymptotic null completions of a simple space-time $(M, \mathbf{g})$, can $\mathscr{I}_{a}^{+}$and $\mathscr{I}_{b}^{+}$be non-homeomorphic?

Of course the foremost challenge is to construct a simple space-time admitting an asymptotic null completion with $\mathscr{I}^{+}$having a topology different from $\mathbb{S}^{2} \times \mathbb{R}$. The subtleties of some Whitehead manifold different from $\mathbb{R}^{3}$ (or a counterexample 
to the Poincare conjecture) must be reflected in the topological structure of any such completion. Moreover the light cones must somehow be oriented so that the entire space-time manifold lies to the past of every point of the strong causality violating region of $\mathscr{I}^{+}$. The necessity for such rich topological and causal behaviour will inevitably impede any attempt at construction. A more reasonable initial goal might therefore be to establish existence.

Acknowledgements. The author has benefitted from many stimulating discussions with Prof. Chris Clarke at the University of Southampton. The early stages of this work were carried out there with the financial support of the Science and Engineering Research Council of Great Britain.

\section{References}

1. Penrose, R.: Zero rest-mass fields including gravitation: Asymptotic behaviour. Proc. R. Soc. A284, 159-203 (1965)

2. Geroch, R.: Space-time structure from a global viewpoint. In: General relativity and cosmology. Sachs, R. K. (ed.) pp. 71-103. Proc. Int. School in Physics 'Enrico Fermi', Course XLVII. New York: Academic Press 1971

3. Hawking, S. W., Ellis, G. F. R.: The large scale structure of space-time. Cambridge: Cambridge University Press 1973

4. Spanier, E.H.: Algebraic topology. New York: McGraw-Hill 1966

5. Penrose, R.: Techniques of differential topology in relativity. Philadelphia: S.I.A.M. 1972

6. Geroch, R.: Asymptotic structure of space-time. In: Asymptotic structure of space-time. Esposito, F. P., Witten, L. (eds.) pp. 1-105. New York: Plenum Press 1977

7. Newman, R. P. A. C., Clarke, C. J. S.: An $\mathbb{R}^{4}$ space-time with a Cauchy surface which is not $\mathbb{R}^{3}$. Class. Quantum Grav. 4 53-60 (1987)

8. Scott, P.: Private communication (1988)

9. Rêgo E., Rourke C.: A proof of the Poincaré conjecture. University of Warwick preprint (1986)

10. Steenrod, N.: The topology of fibre bundles. Princeton, N.J.: Princeton University Press 1951

11. Hempel, J.: 3-Manifolds. Princeton, NJ: Princeton University Press 1976

12. Freedman, M. H.: The topology of four-dimensional manifolds. J. Diff. Geom. 37, 357-453 (1982)

13. Karoubi, M.: K-Theory: an introduction. Berlin, Heidelberg, New York: Springer 1978

14. McMillan, D. R. Jr.: Cartesian products of contractible open 3-manifolds. Bull. Am. Math. Soc. 67, 510-514 (1961)

15. Kister, J. M., McMillan, D. R. Jr.: Locally euclidean factors of $E^{4}$ which cannot be embedded in $E^{3}$. Ann. Math. 76, 541-546 (1962)

16. Kirby, R. C., Siebenmann, L. C.: Foundational essays on topological manifolds, smoothings and triangulations. Ann. Math. Stud. No. 88. Princeton, NJ: Princeton University Press 1977

17. Hudson, J. E. P.: Piecewise linear topology. New York: W. A. Benjamin 1969

Communicated by S.-T. Yau

Received April 4, 1988; in revised form November 8, 1988 